\title{
Oxidative Deconstruction of Azetidinols to $\alpha$-Amino Ketones
}

\section{Supporting Information}

Robert-Cristian Raclea, Philipp Natho, Lewis A. T. Allen, Andrew J. P. White \& Philip J. Parsons*

Department of Chemistry, Imperial College London, Molecular Sciences Research Hub, W12 0BZ, London, UK

Corresponding Author: Philip J. Parsons

E-Mail: $\quad$ p.parsons@imperial.ac.uk

ORCID: $\quad$ Philip J. Parsons: 0000-0002-9158-4034

\section{Table of Contents}

${ }^{1}$ H NMR Features of $\alpha$-Amino Ketones $2 a-p$...................................... S2

Full Optimization Studies....................................................................... S3

${ }^{1} \mathrm{H},{ }^{13} \mathrm{C}$ and ${ }^{19} \mathrm{~F}$ NMR Spectra ................................................................... S4

Deuterated Solvent Experiment.................................................................. S56 


\section{${ }^{1}$ H NMR Features of $\alpha$-Amino Ketones 2a-p}

A large proportion of the $\alpha$-amino ketones generated through this methodology are obtained as a mixture of a hydrogen-bonded and a non-hydrogen-bonded isomer. In the presence of an omethoxy substituent, the separation and individual assignment of the two isomeric ketones $(\mathbf{2} \mathbf{j}$, j') was achieved. Key differences are presented in Figure 1. In the ${ }^{1} \mathrm{H}$ NMR spectrum of $\mathbf{2} \mathbf{j}$, the $\alpha$-carbonyl $\mathrm{CH}_{2}$ group (shown in red) appears as a doublet $(J=4.8 \mathrm{~Hz}$ ) at $4.61 \mathrm{ppm}$, whereas in $\mathbf{2} \mathbf{j}$ ' it appears as a singlet at $4.97 \mathrm{ppm}$ (Figure S1). The NH proton (shown in blue) shifts even more drastically, from $5.63 \mathrm{ppm}$ in $\mathbf{2} \mathbf{j}$ to $9.31 \mathrm{ppm}$ in $\mathbf{2 \mathbf { j }}$ '.

Figure S1: Partial ${ }^{1} \mathrm{H}$ NMR spectra of $2 \mathrm{j}$ (a) and $2 \mathrm{j}$ ' (b)

a)
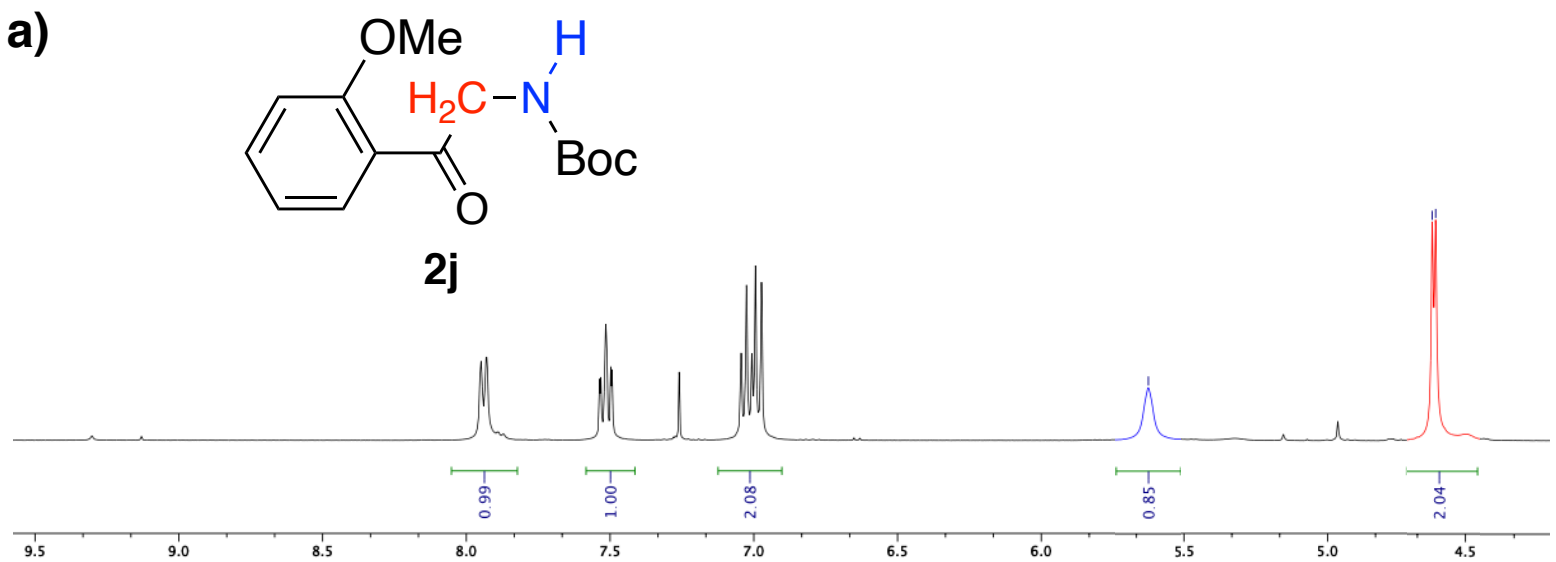

b)

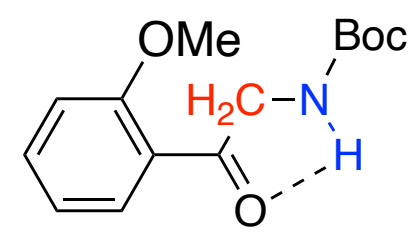

2j'

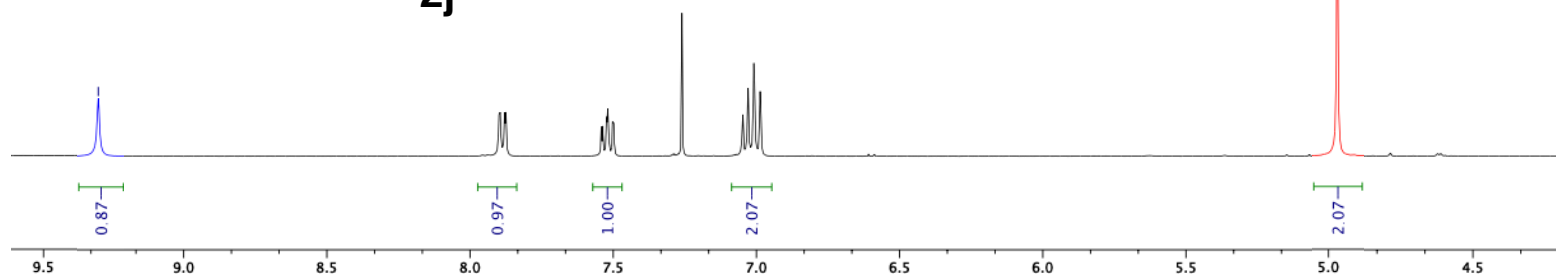




\section{Full Optimization Studies}

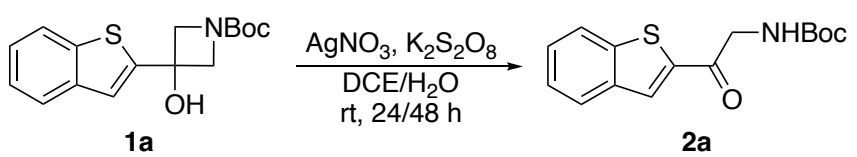

\begin{tabular}{|c|c|c|c|}
\hline entry & variation from standard conditions ${ }^{a}$ & $\begin{array}{l}\mathrm{RSM}^{b} \\
{[\%]}\end{array}$ & $\begin{array}{l}\text { yield } \\
{[\%]}\end{array}$ \\
\hline 1 & DCM instead of DCE & 16 & 15 \\
\hline 2 & DCM instead of DCE, Ts instead of Boc & 26 & 5 \\
\hline 3 & MeCN instead of DCE & 65 & 5 \\
\hline 4 & DMF instead of DCE & 91 & trace \\
\hline 5 & none & 10 & 32 \\
\hline 6 & reaction open to atmosphere & trace & trace \\
\hline 7 & non-degassed deionized water used & 19 & 21 \\
\hline 8 & $\mathrm{AgBF}_{4}$ instead of $\mathrm{AgNO}_{3}$ & 36 & 10 \\
\hline 9 & $\mathrm{Na}_{2} \mathrm{~S}_{2} \mathrm{O}_{8}$ instead of $\mathrm{K}_{2} \mathrm{~S}_{2} \mathrm{O}_{8}$ & trace & 22 \\
\hline 10 & $35^{\circ} \mathrm{C}$ instead of $\mathrm{rt}$ & 22 & 26 \\
\hline 11 & $50{ }^{\circ} \mathrm{C}$ instead of $\mathrm{rt}$ & 40 & 18 \\
\hline 12 & $0.5 \mathrm{~mL} \mathrm{DCE} / \mathrm{H}_{2} \mathrm{O}$ & 44 & 11 \\
\hline 13 & $2 \mathrm{~mL}$ DCE$/ \mathrm{H}_{2} \mathrm{O}$ & 33 & 22 \\
\hline 14 & $1 \mathrm{~mL} \mathrm{DCE} / \mathrm{H}_{2} \mathrm{O}(9: 1)$ & 56 & 6 \\
\hline 15 & $1 \mathrm{~mL} \mathrm{DCE} / \mathrm{H}_{2} \mathrm{O}(7: 3)$ & 50 & 7 \\
\hline 16 & $1 \mathrm{~mL} \mathrm{DCE} / \mathrm{H}_{2} \mathrm{O}(3: 7)$ & 20 & 18 \\
\hline 17 & $1 \mathrm{~mL} \mathrm{DCE} / \mathrm{H}_{2} \mathrm{O}(1: 9)$ & 44 & 8 \\
\hline 18 & 0.35 equiv $\mathrm{AgNO}_{3}$ & 21 & 20 \\
\hline 19 & 0.5 equiv $\mathrm{AgNO}_{3}$ & 27 & 23 \\
\hline 20 & 1 equiv $\mathrm{AgNO} 3$ & 45 & 11 \\
\hline 21 & 4 equiv of $\mathrm{AgNO} 3 \&\left(\mathrm{NH}_{4}\right)_{2} \mathrm{~S}_{2} \mathrm{O}_{8}, 1 \mathrm{~mL}$ acetone $/ \mathrm{H}_{2} \mathrm{O}(1: 9)$ & 85 & trace \\
\hline 22 & 4 equiv of $\mathrm{AgNO} 3 \&\left(\mathrm{NH}_{4}\right)_{2} \mathrm{~S}_{2} \mathrm{O}_{8}$, acetone instead of DCE & trace & 25 \\
\hline 23 & 4 equiv of $\mathrm{AgNO}_{3} \& \mathrm{~K}_{2} \mathrm{~S}_{2} \mathrm{O}_{8}$ & 5 & 27 \\
\hline 24 & 4 equiv of $\mathrm{AgNO} 3 \&\left(\mathrm{NH}_{4}\right)_{2} \mathrm{~S}_{2} \mathrm{O}_{8}$ & 11 & 27 \\
\hline 25 & supplementary 0.2 equiv $\mathrm{AgNO}_{3}$ added after $2 \mathrm{~h}, 4 \mathrm{~h}$ and $6 \mathrm{~h}$ & 19 & 16 \\
\hline 26 & $\begin{array}{l}1 \text { equiv } \mathrm{K}_{2} \mathrm{~S}_{2} \mathrm{O}_{8} \text {, supplementary } 0.2 \text { equiv } \mathrm{AgNO}_{3} \text { and } 0.7 \text { equiv } \mathrm{K}_{2} \mathrm{~S}_{2} \mathrm{O}_{8} \text { added after } \\
2 \mathrm{~h}, 4 \mathrm{~h}, 6 \mathrm{~h} \text { and } 8 \mathrm{~h}\end{array}$ & 38 & 10 \\
\hline 27 & second portion of oxidant/co-oxidant added after $12 \mathrm{~h}$ & 31 & 18 \\
\hline 28 & $48 \mathrm{~h}$, second portion of oxidant/co-oxidant added after $24 \mathrm{~h}$ & 15 & 35 \\
\hline 29 & $\begin{array}{l}116 \mathrm{~h} \text {, second portion of oxidant/co-oxidant added after } 24 \mathrm{~h} \text {, third portion of } \\
\text { oxidant/co-oxidant added after } 48 \mathrm{~h}\end{array}$ & trace & 40 \\
\hline 30 & $48 \mathrm{~h}, 4$ equiv $\mathrm{K}_{2} \mathrm{~S}_{2} \mathrm{O}$, second portion of oxidant/co-oxidant added after $24 \mathrm{~h}$ & trace & 40 \\
\hline 31 & $48 \mathrm{~h}, 4$ equiv $\left(\mathrm{NH}_{4}\right)_{2} \mathrm{~S}_{2} \mathrm{O} 8$, second portion of oxidant/co-oxidant added after $24 \mathrm{~h}$ & trace & 38 \\
\hline
\end{tabular}

${ }^{a}$ Standard conditions: 1a $(0.30 \mathrm{mmol}), \mathrm{AgNO}_{3}$ (0.2 equiv) and $\mathrm{K}_{2} \mathrm{~S}_{2} \mathrm{O}_{8}$ (3 equiv) in $1 \mathrm{~mL}$ DCE/ $\mathrm{H}_{2} \mathrm{O}$ (1:1), rt, 24 h. ${ }^{b}$ Recovered starting material. 


\section{${ }^{1} \mathrm{H},{ }^{13} \mathrm{C}$ and ${ }^{19} \mathrm{~F}$ NMR Spectra}

tert-Butyl 3-(benzo[b]thiophen-2-yl)-3-hydroxyazetidine-1-carboxylate, 1a

${ }^{1} \mathrm{H}$ NMR $(400 \mathrm{MHz}$, Chloroform- $d$ ) 87.81 (ddd, $J=7.4,1.7,0.7 \mathrm{~Hz}, 1 \mathrm{H}), 7.77-7.70(\mathrm{~m}, 1 \mathrm{H})$, $7.40-7.30(\mathrm{~m}, 2 \mathrm{H}), 7.30(\mathrm{~s}, 1 \mathrm{H}), 4.32(\mathrm{~d}, J=9.3 \mathrm{~Hz}, 2 \mathrm{H}), 4.23(\mathrm{dd}, J=9.3,1.0 \mathrm{~Hz}, 2 \mathrm{H}), 3.40$ $(\mathrm{s}, 1 \mathrm{H}), 1.46(\mathrm{~s}, 9 \mathrm{H})$
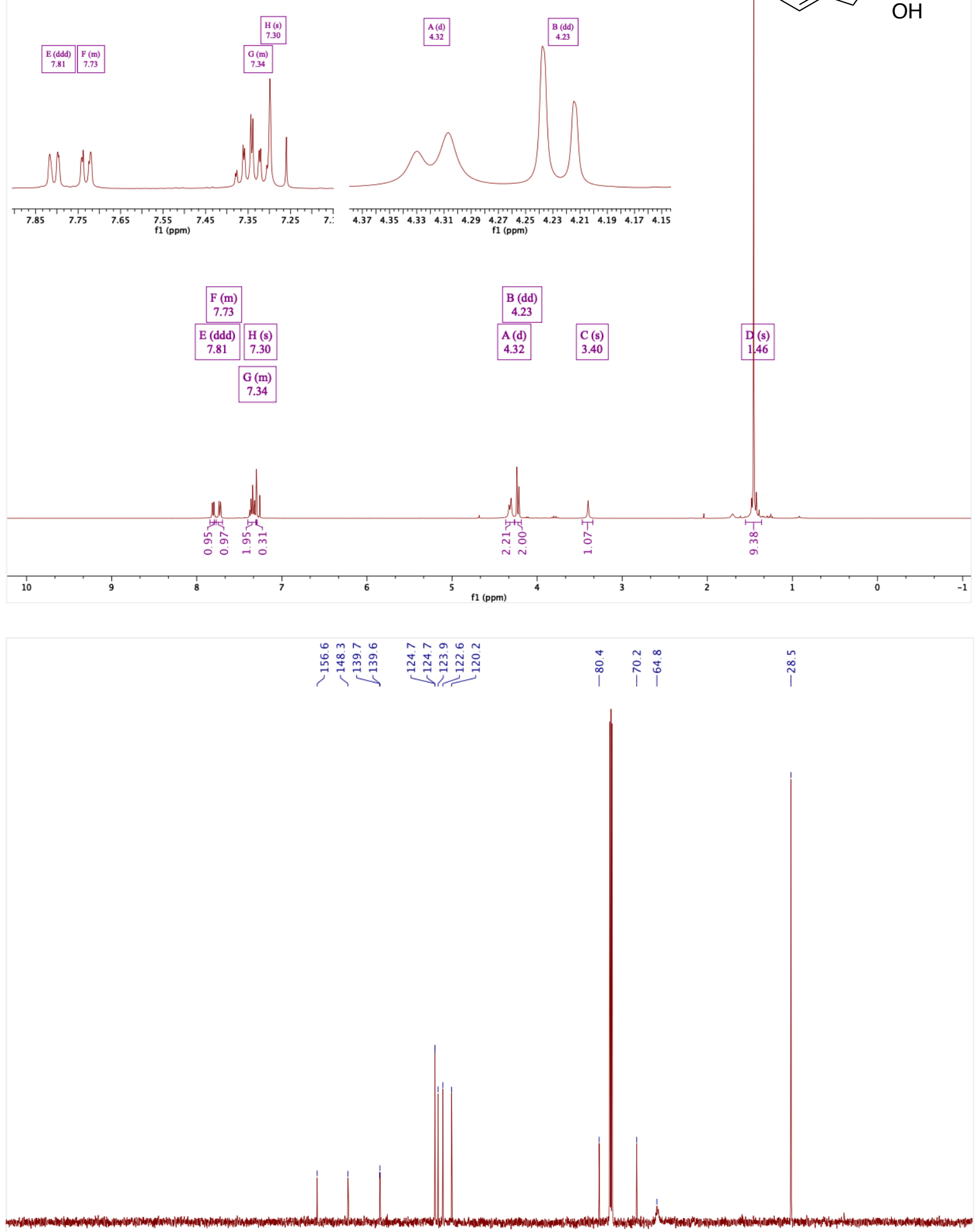

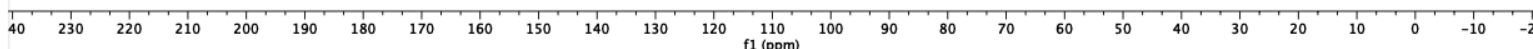




\section{3-(Benzo[b]thiophen-2-yl)-1-tosylazetidin-3-ol}

'H NMR ( 400 MHz, Chloroform- $d$ ) $87.82-7.72(\mathrm{~m}, 3 \mathrm{H}), 7.70-7.62(\mathrm{~m}, 1 \mathrm{H}), 7.38$ (d, $J=8.1$

.
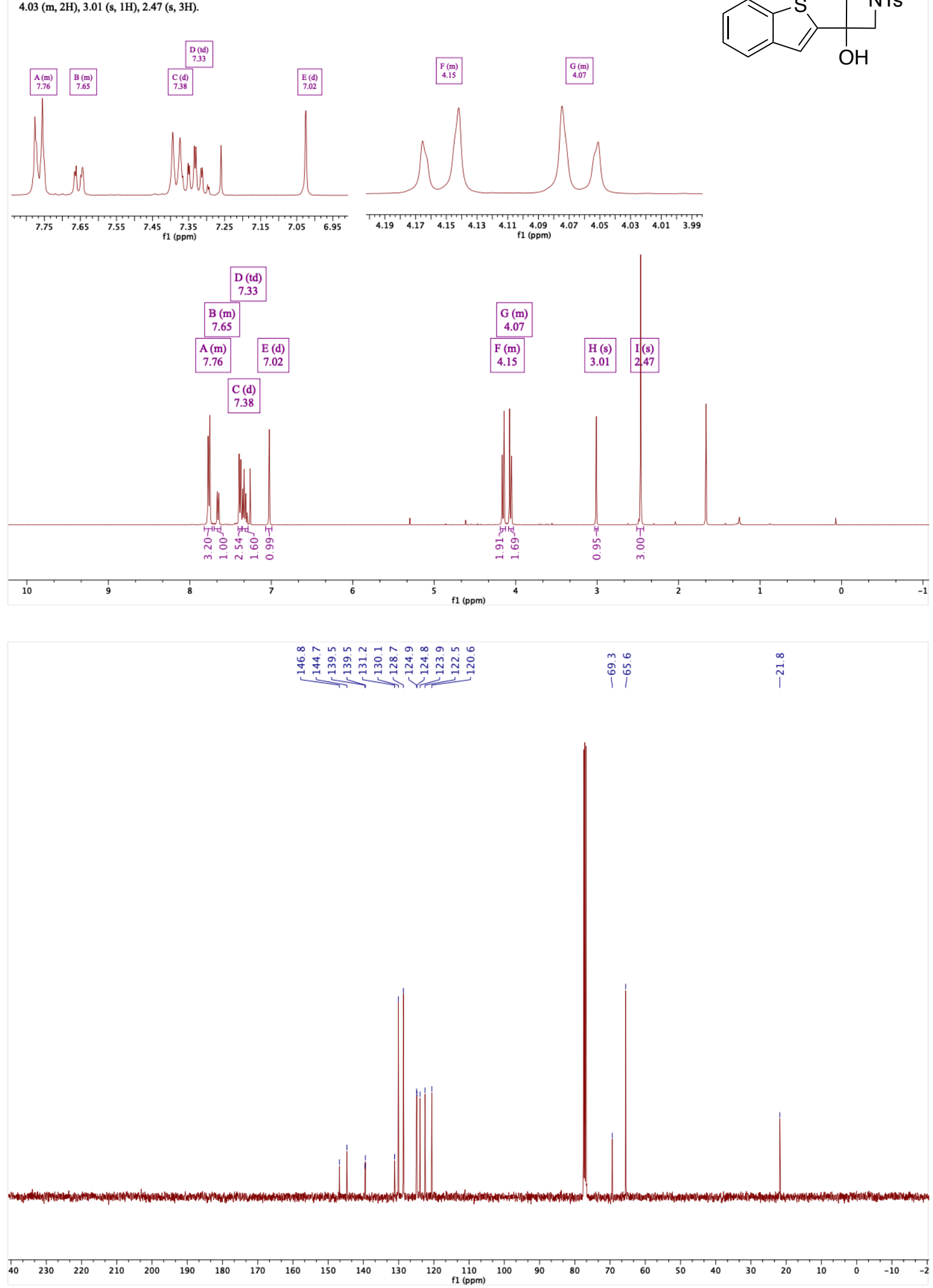
tert-Butyl 3-hydroxy-3-phenylazetidine-1-carboxylate, $1 \mathrm{~b}$
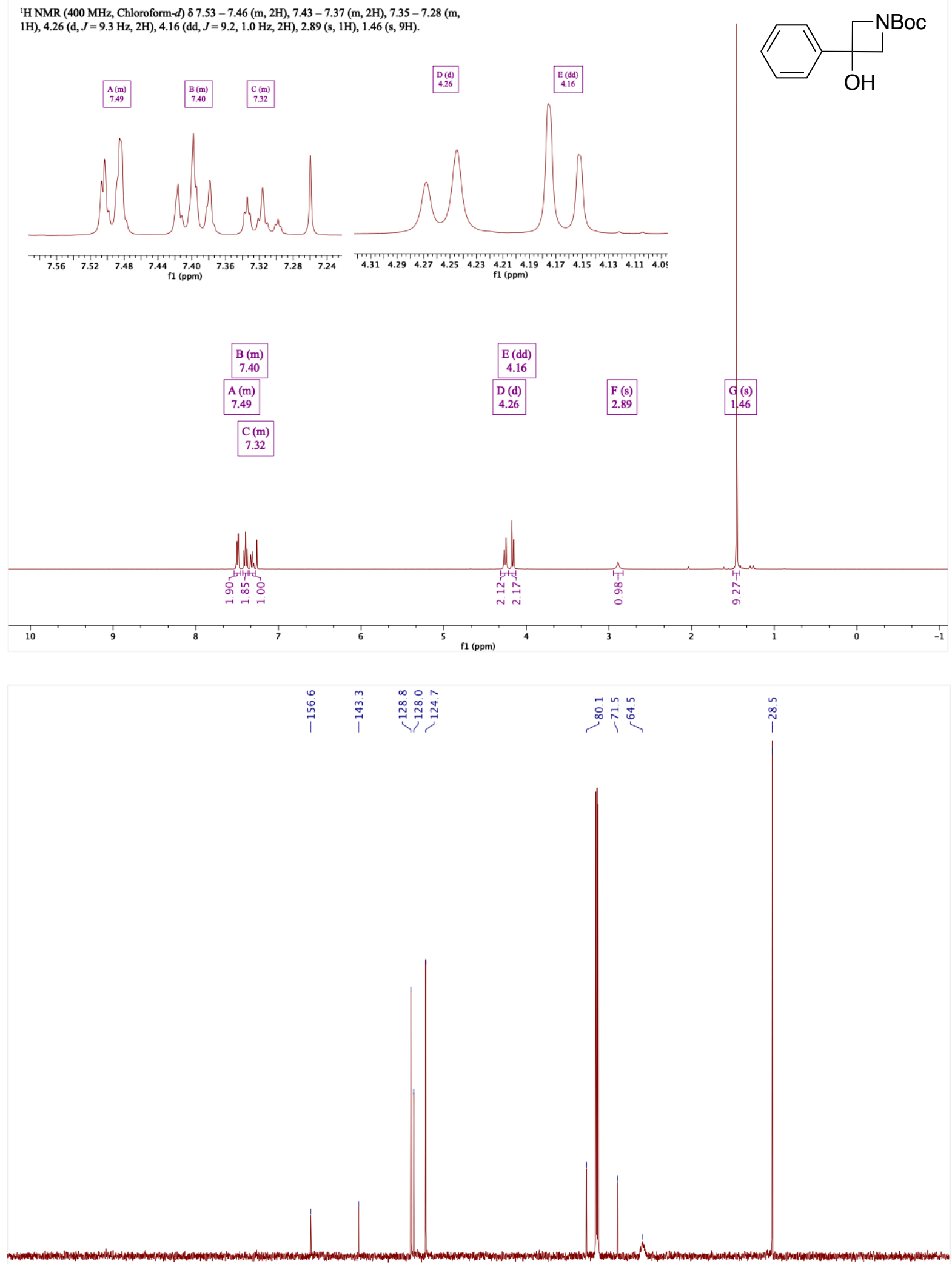

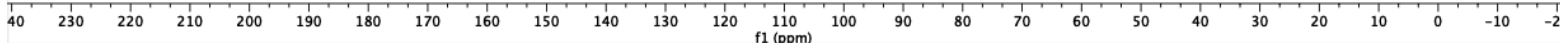


tert-Butyl 3-(4-fluorophenyl)-3-hydroxyazetidine-1-carboxylate, 1c

${ }^{1} \mathrm{H}$ NMR $(400 \mathrm{MHz}$, Chloroform- $d) \delta 7.53-7.43(\mathrm{~m}, 2 \mathrm{H}), 7.07$ (t, $\left.J=8.7 \mathrm{~Hz}, 2 \mathrm{H}\right), 4.22(\mathrm{~d}, J=$ $9.3 \mathrm{~Hz}, 2 \mathrm{H}), 4.16(\mathrm{~d}, J=9.2 \mathrm{~Hz}, 2 \mathrm{H}), 2.87(\mathrm{~s}, 1 \mathrm{H}), 1.45(\mathrm{~s}, 9 \mathrm{H})$

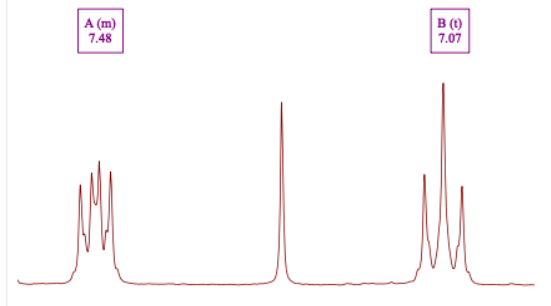

$\begin{array}{lllllllllllllll}7.55 & 7.50 & 7.45 & 7.40 & 7.35 & 7.30 & 7.25 & 7.20 & 7.15 & 7.10 & 7.05 & 7.00\end{array}$
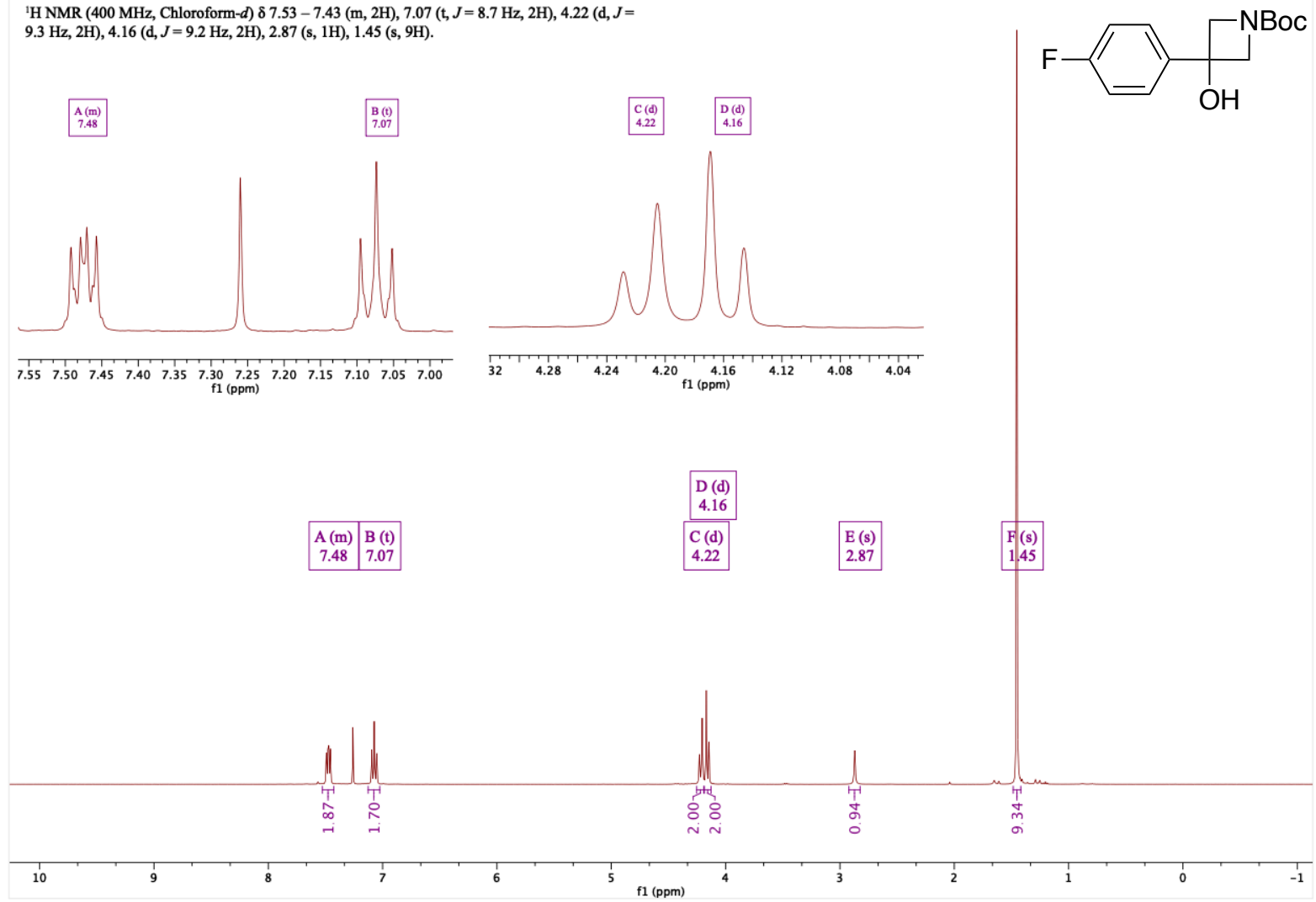

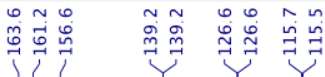

${ }^{13} \mathrm{C}$ NMR (101 MHz, Chloroform- $d$ ) $\delta 162.42$ (d, $\left.J=247.1 \mathrm{~Hz}\right), 156.58,139.18$ (d, $\left.J=2.5 \mathrm{~Hz}\right)$ $126.60(\mathrm{~d}, J=8.3 \mathrm{~Hz}), 115.62(\mathrm{~d}, J=21.6 \mathrm{~Hz}), 80.24,71.13,64.66,28.51$

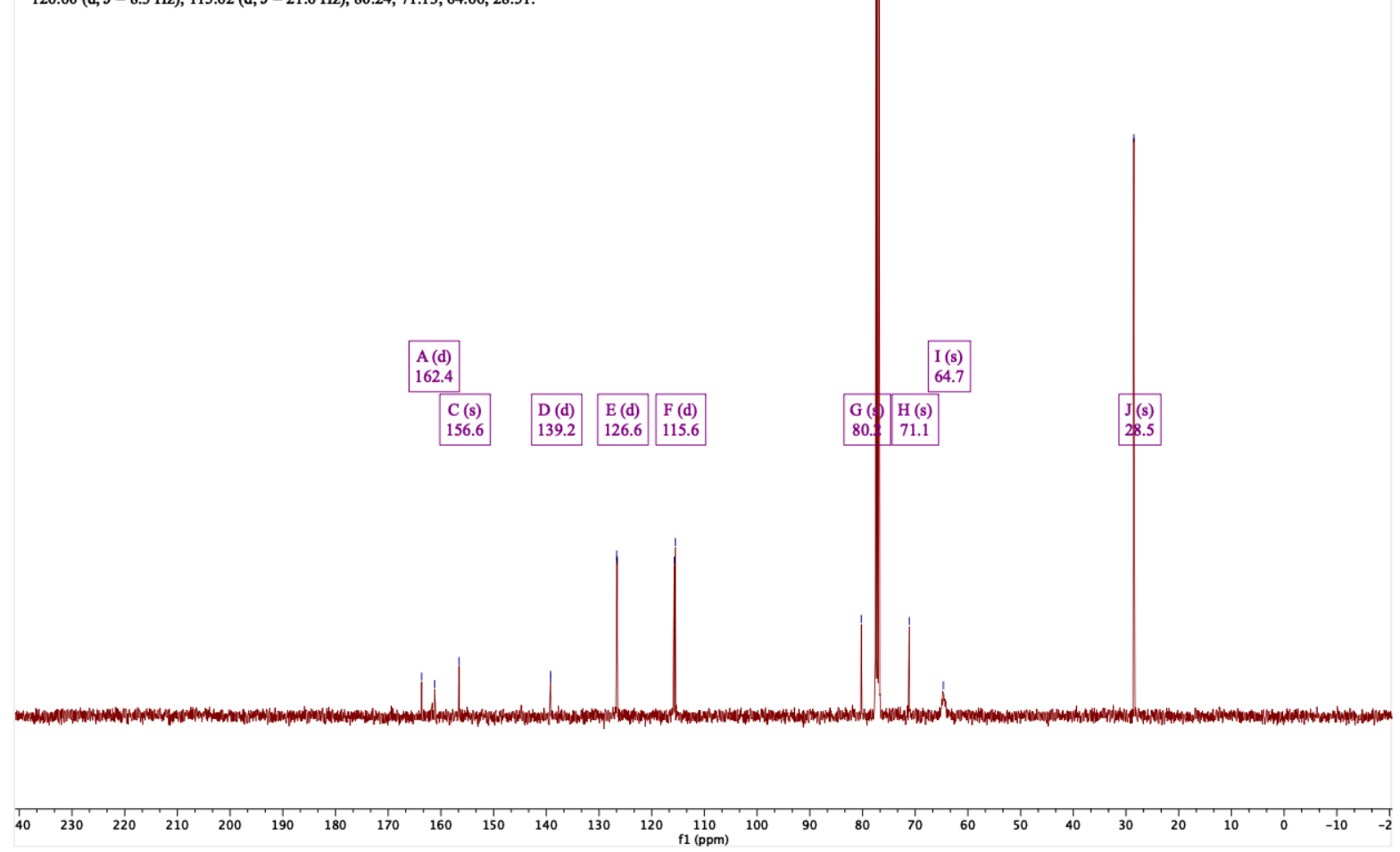




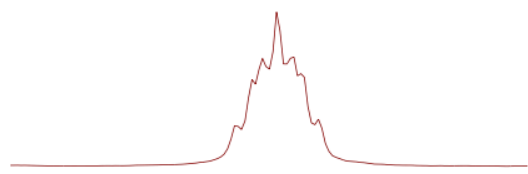

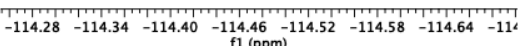

$A(p)$
-114.48

$\mid$

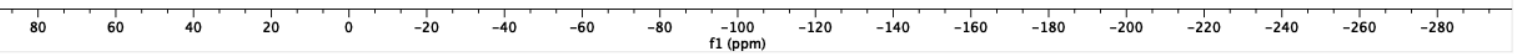


tert-Butyl 3-(3-fluorophenyl)-3-hydroxyazetidine-1-carboxylate, 1d

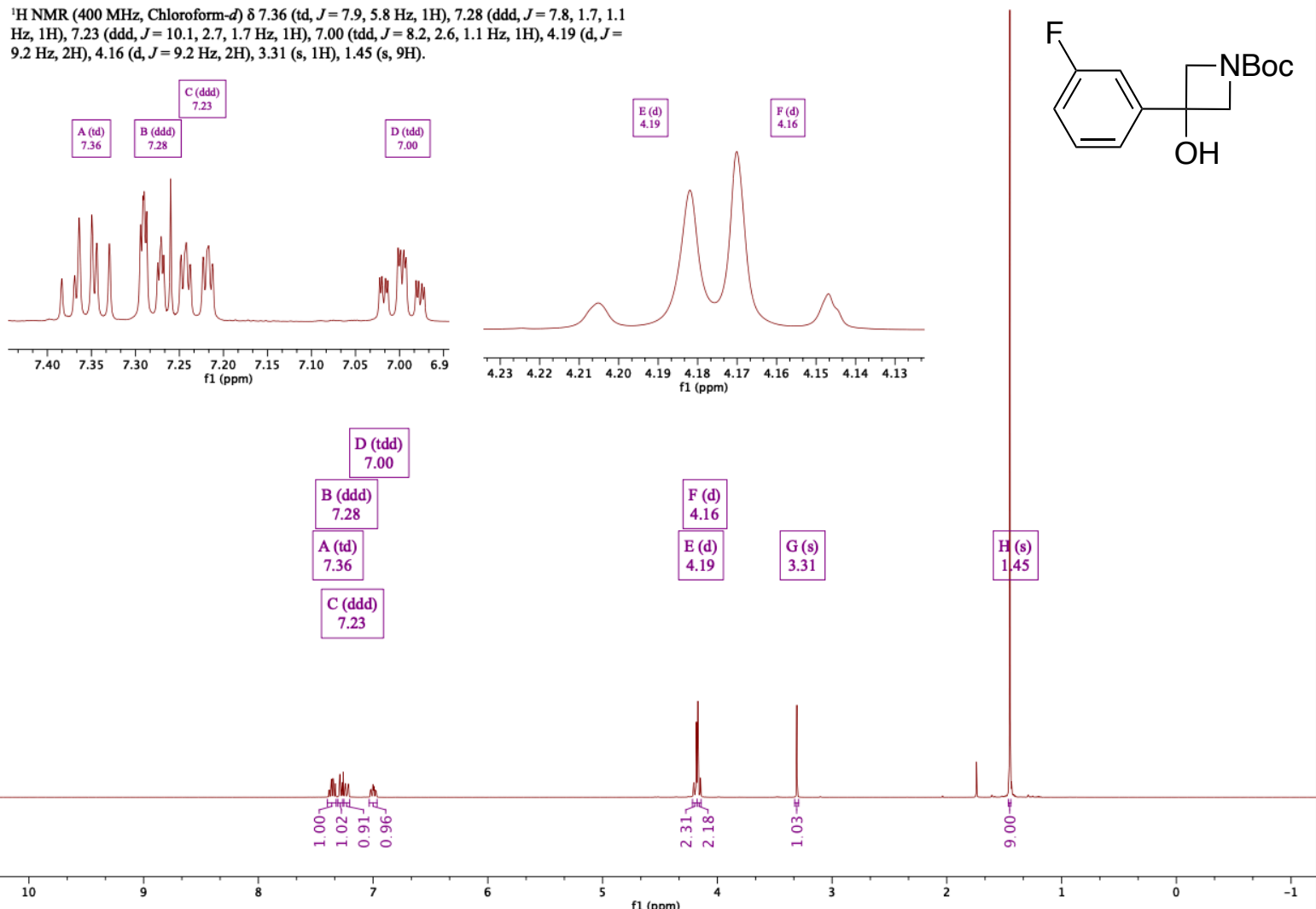

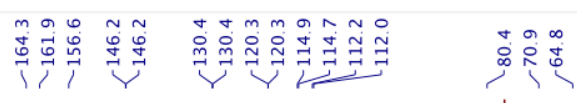

${ }^{13} \mathrm{C}$ NMR (101 MHz, Chloroform- $d$ ) $\delta 163.12$ (d, $\left.J=246.4 \mathrm{~Hz}\right), 156.60,146.21$ (d, $\left.J=6.9 \mathrm{~Hz}\right)$, $130.39(\mathrm{~d}, J=8.0 \mathrm{~Hz}), 120.27$ (d, $J=2.9 \mathrm{~Hz}), 114.82(\mathrm{~d}, J=21.0 \mathrm{~Hz}), 112.07(\mathrm{~d}, J=22.7 \mathrm{~Hz})$, $80.36,70.92,64.75,28.50$.

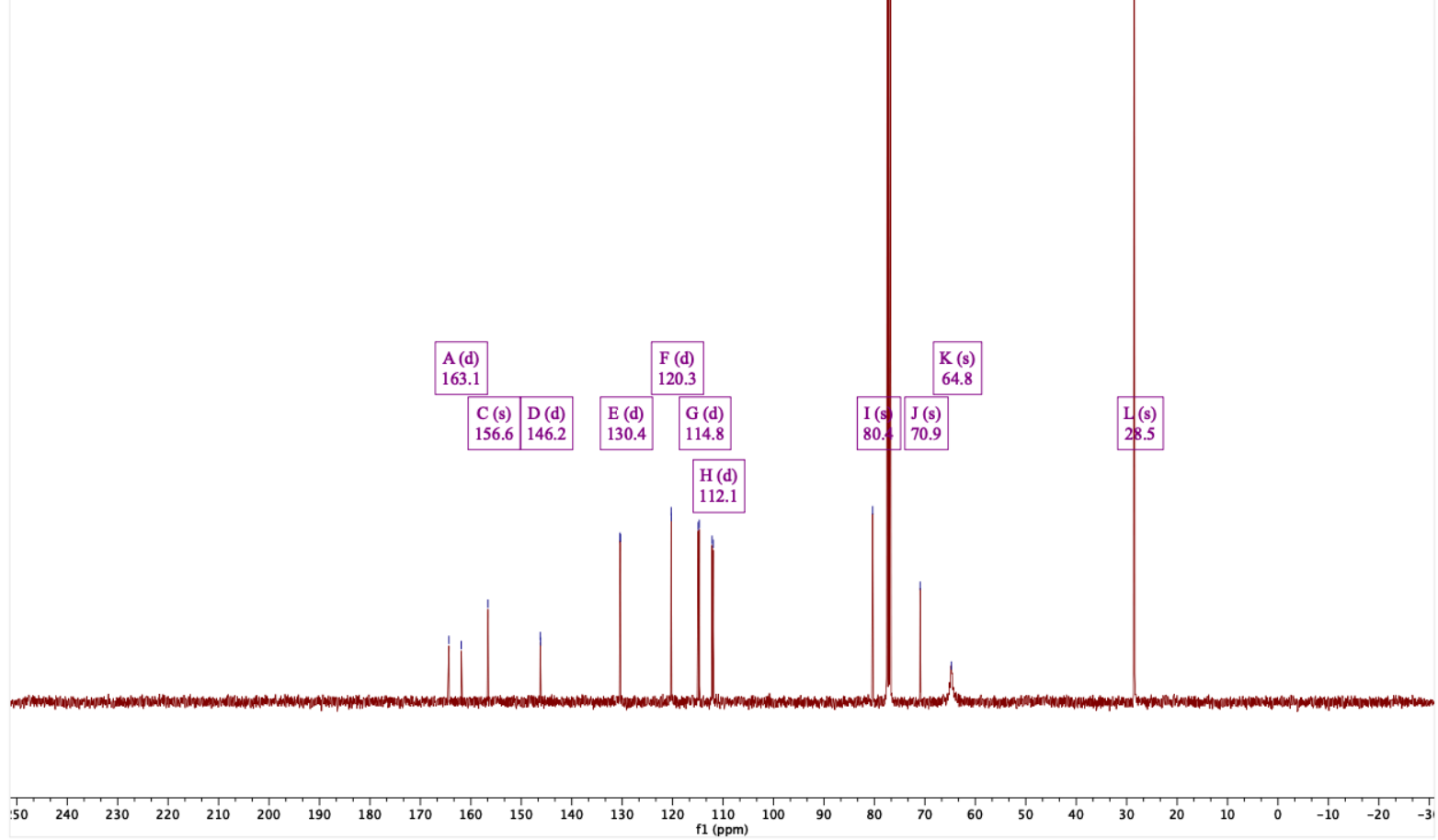


${ }^{19} \mathrm{~F}$ NMR $(377 \mathrm{MHz}$, Chloroform $-d) \delta-112.29(\mathrm{q}, J=8.5 \mathrm{~Hz})$.

워요

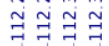

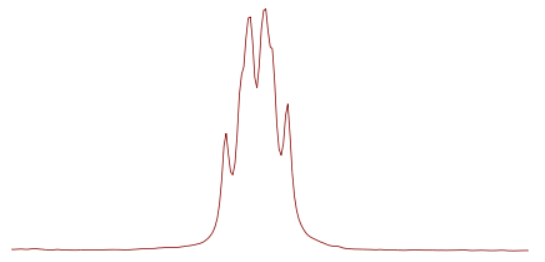

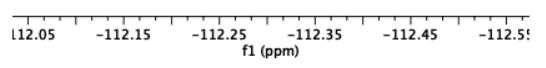

\begin{tabular}{|c|}
\hline $\mathbf{A}(\mathfrak{q})$ \\
-112.29
\end{tabular}

50

$\begin{array}{r}1 \\ -60\end{array}-70$

$-70 \quad-80$

$-90$

$-100$

$-110$

-120 f1 (ppm) -130

$-160$

$-170$

$-190$ 
tert-Butyl 3-(2-fluorophenyl)-3-hydroxyazetidine-1-carboxylate, 1e

${ }^{1} \mathrm{H}$ NMR ( $400 \mathrm{MHz}$, Chloroform- $d$ ) 87.38 (td, $J=7.8,1.8 \mathrm{~Hz}, 1 \mathrm{H}$ ), 7.32 (dddd, $J=8.2,7.2$ $5.2,1.8 \mathrm{~Hz}, 1 \mathrm{H}), 7.16(\mathrm{td}, J=7.6,1.2 \mathrm{~Hz}, 1 \mathrm{H}), 7.09(\mathrm{ddd}, J=11.2,8.2,1.2 \mathrm{~Hz}, 1 \mathrm{H}), 4.42(\mathrm{dt}, J$

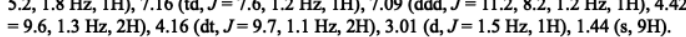

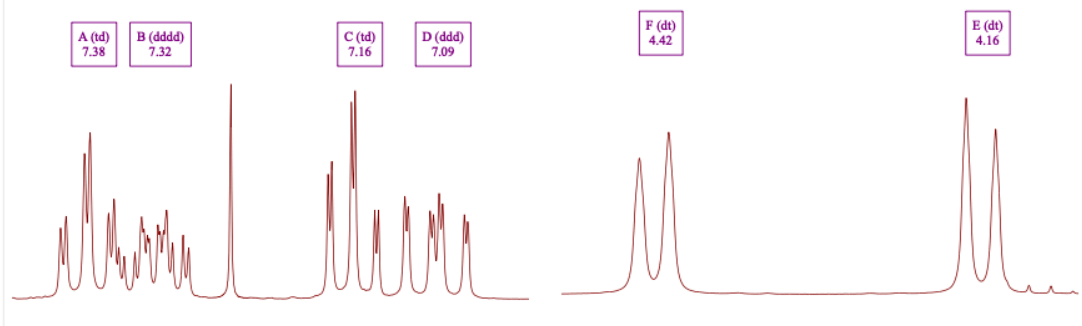

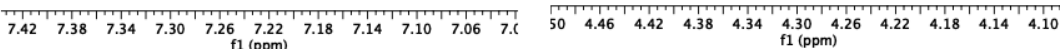

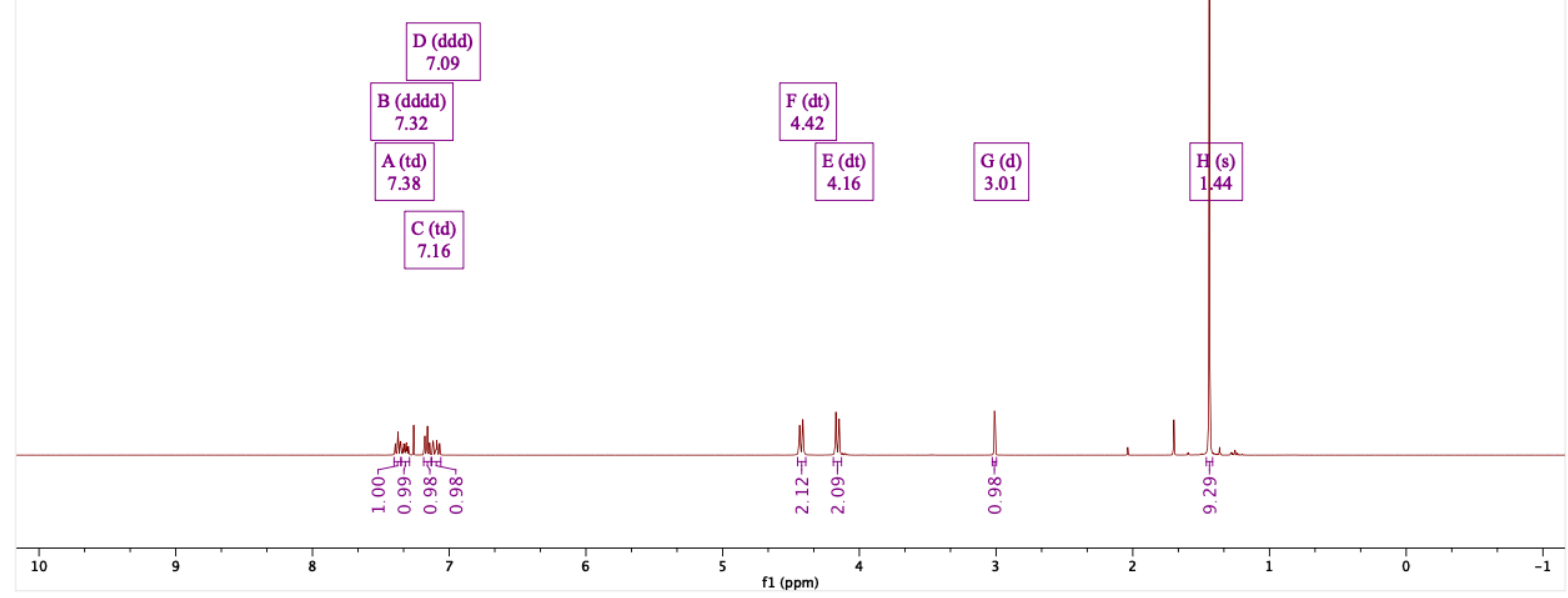

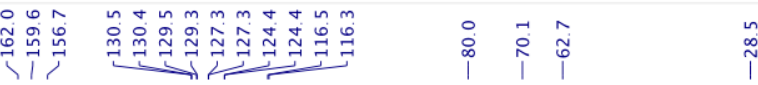

${ }^{13} \mathrm{C}$ NMR (101 MHz, Chloroform- $d$ ) $\delta 160.81$ (d, $\left.J=247.4 \mathrm{~Hz}\right), 156.65,130.41$ (d, $J=8.5 \mathrm{~Hz}$ ), $129.41(\mathrm{~d}, J=13.0 \mathrm{~Hz}), 127.32(\mathrm{~d}, J=4.1 \mathrm{~Hz}), 124.40(\mathrm{~d}, J=3.5 \mathrm{~Hz}), 116.40(\mathrm{~d}, J=21.5 \mathrm{~Hz})$, 79.98, $70.07,62.74,28.51$

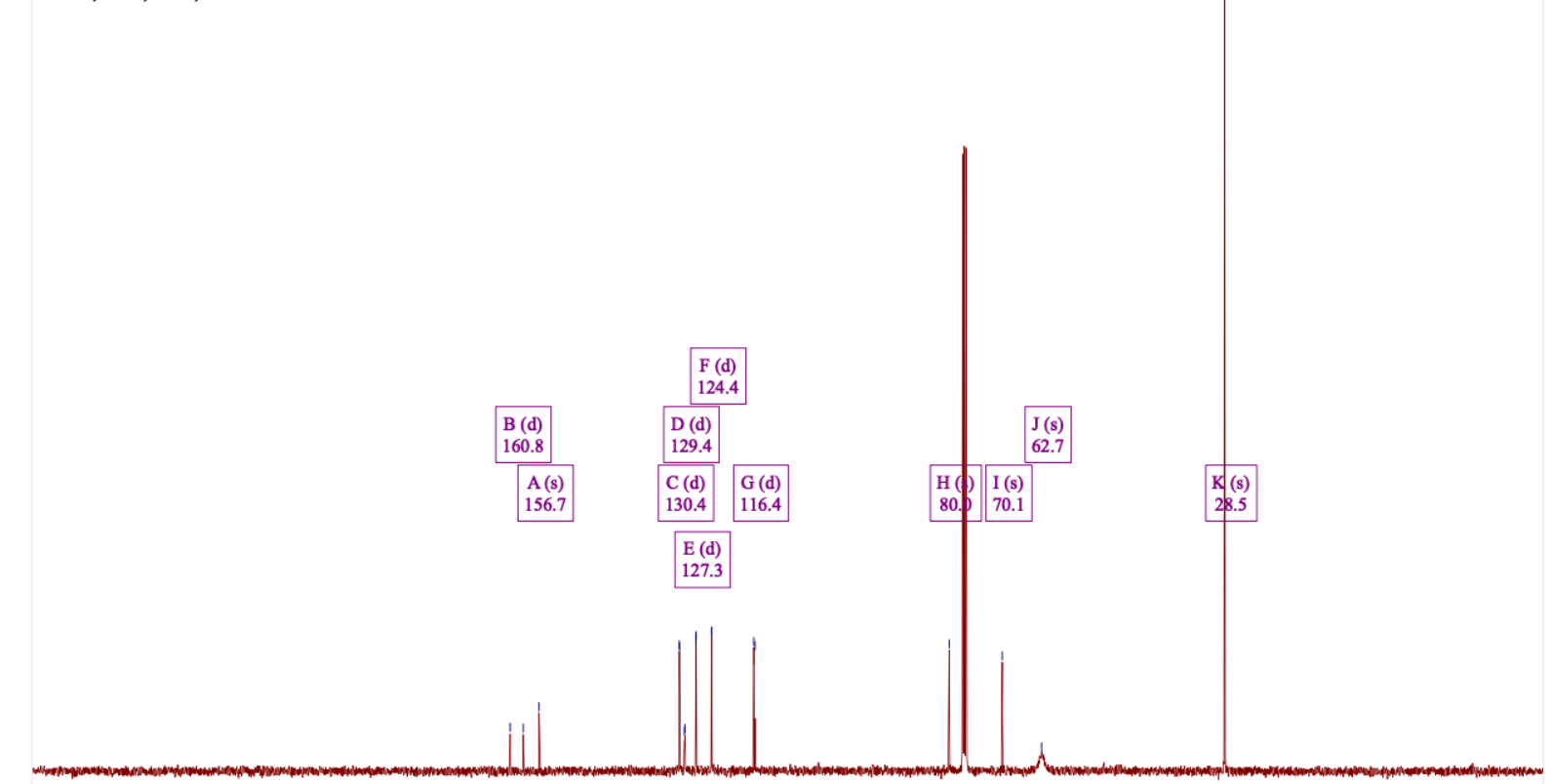


${ }^{19} \mathrm{~F}$ NMR $(377 \mathrm{MHz}$, Chloroform- $d$ ) $\delta-115.78(\mathrm{dt}, J=11.8,6.0 \mathrm{~Hz})$.

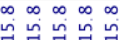

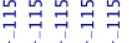

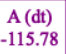

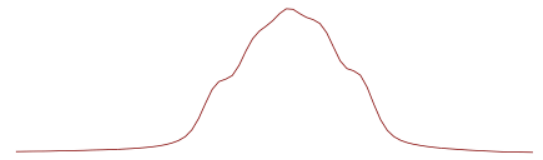

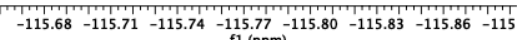
(1) (ppm)

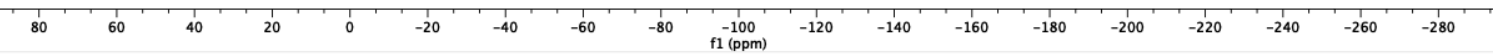


tert-Butyl 3-(2,6-difluorophenyl)-3-hydroxyazetidine-1-carboxylate, if
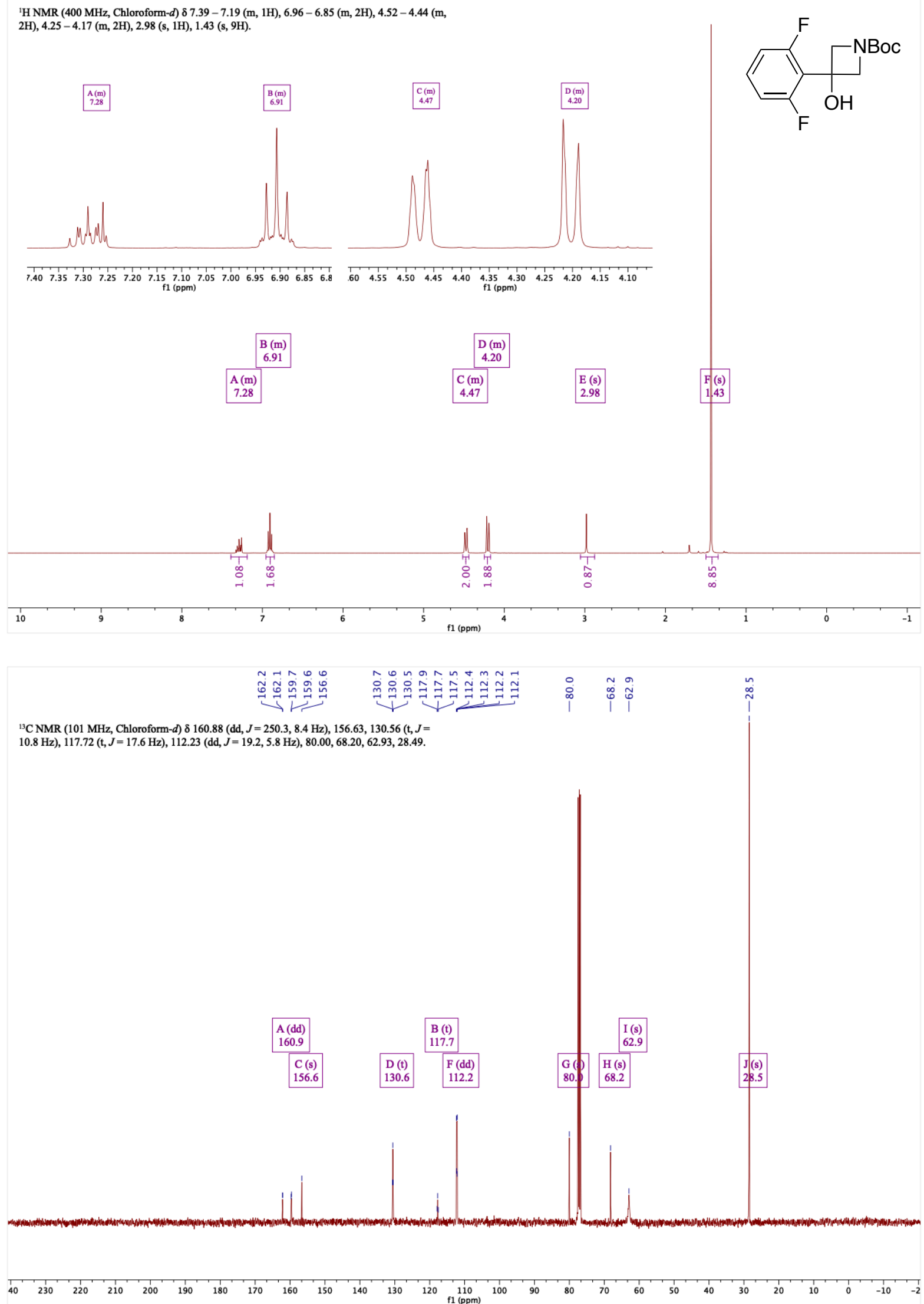


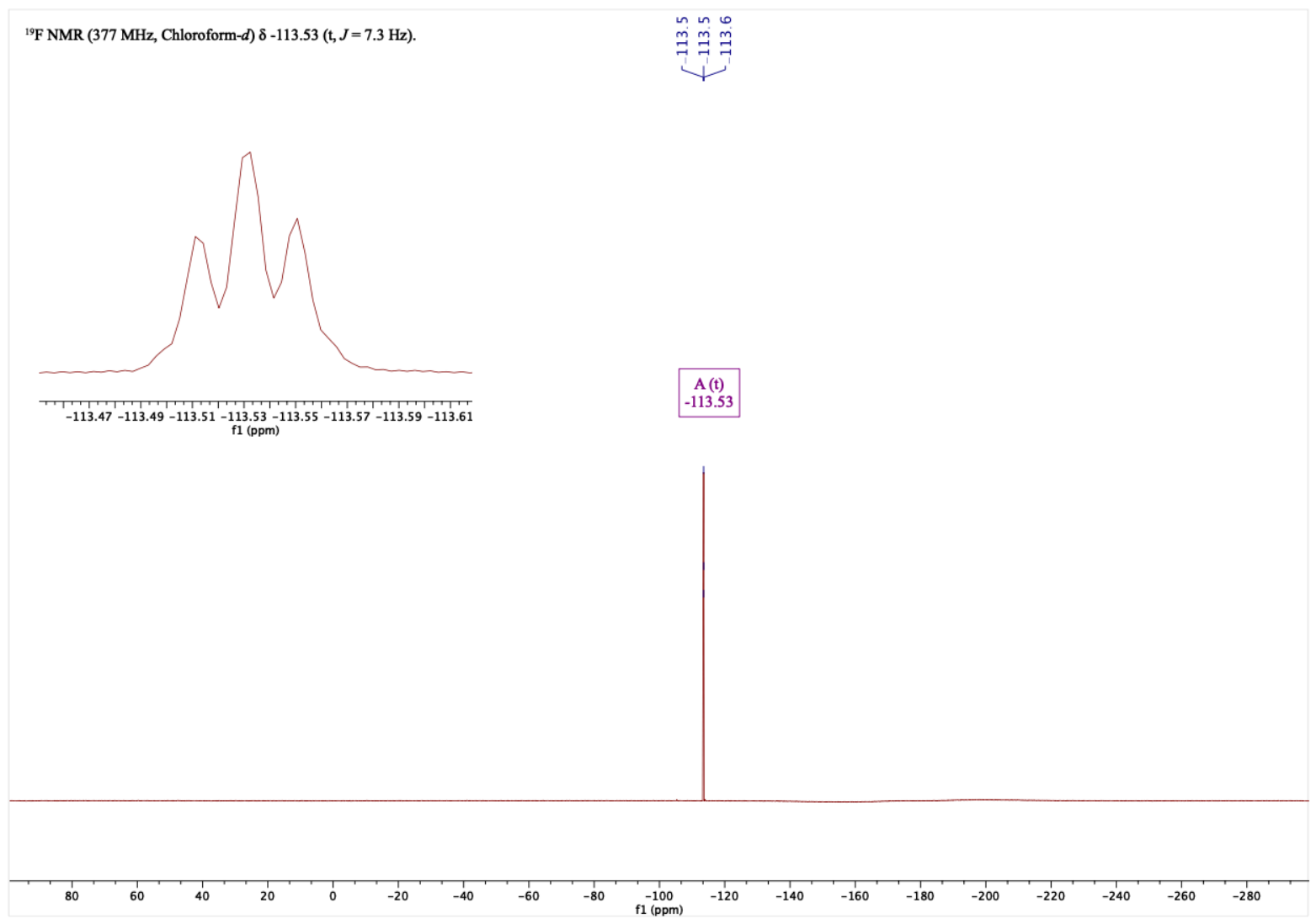

S14 
tert-Butyl 3-hydroxy-3-(4-methoxyphenyl)azetidine-1-carboxylate, $1 \mathrm{~g}$
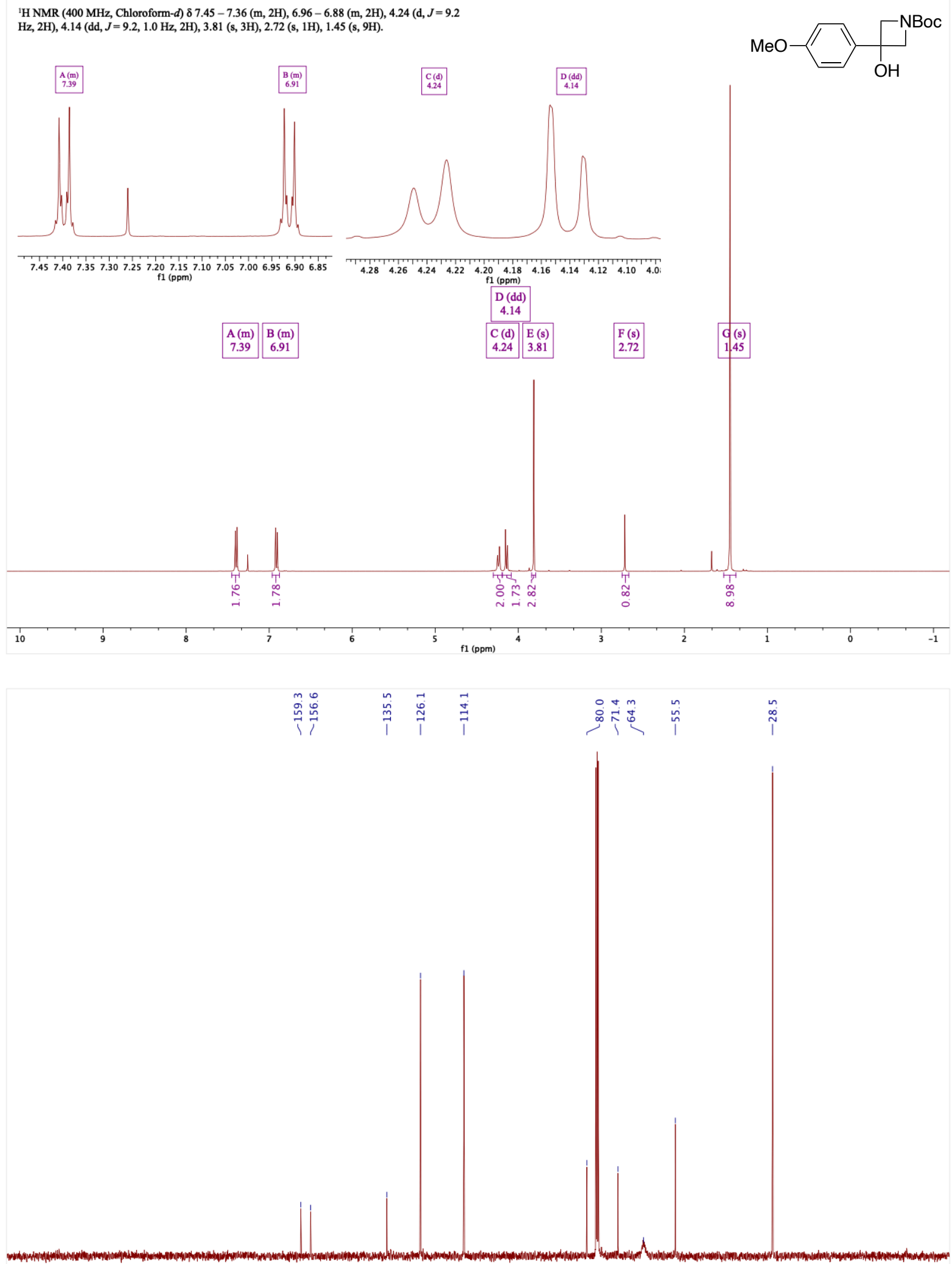

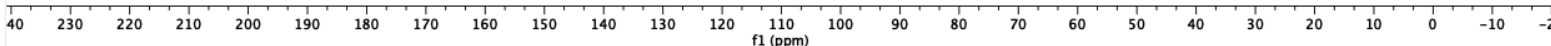


tert-Butyl 3-hydroxy-3-(3-methoxyphenyl)azetidine-1-carboxylate, $1 \mathrm{~h}$

'H NMR ( $400 \mathrm{MHz}$, Chloroform- $d$ ) $\delta 7.31$ (t, $J=7.9 \mathrm{~Hz}, 1 \mathrm{H}$ ), 7.07 (ddd, $J=7.7,1.8,0.9 \mathrm{~Hz}$ $1 \mathrm{H}, 7.04(\mathrm{t}, J=2.1 \mathrm{~Hz}, 1 \mathrm{H}), 6.85$ (ddd, $J=8.2,2.6,0.9 \mathrm{~Hz}, 1 \mathrm{H}), 4.24(\mathrm{~d}, J=9.3 \mathrm{~Hz}, 2 \mathrm{H}), 4.14$ $(\mathrm{dd}, J=9.3,1.0 \mathrm{~Hz}, 2 \mathrm{H}), 3.82(\mathrm{~s}, 3 \mathrm{H}), 2.95(\mathrm{~s}, 1 \mathrm{H}), 1.45(\mathrm{~s}, 9 \mathrm{H})$.
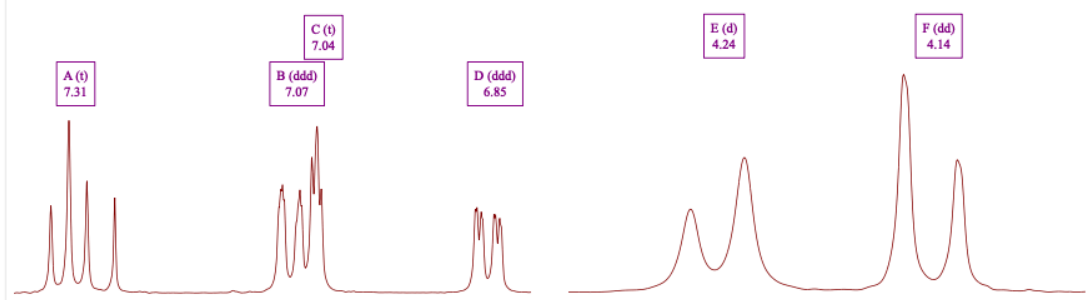

$\mathrm{MeO}$

$\begin{array}{llllllllllllllll}7.35 & 7.30 & 7.25 & 7.20 & 7.15 & 7.10 & 7.05 & 7.00 & 6.95 & 6.90 & 6.85\end{array}$

$\begin{array}{llllllllllll}4.28 & 4.26 & 4.24 & 4.22 & 4.20 & 4.18 & 4.16 & 4.14 & 4.12 & 4.10 & 4 . C\end{array}$

F(dd)

\begin{tabular}{|c|c|}
\hline $\begin{array}{c}\text { B (ddd) } \\
7.07\end{array}$ \\
\hline A (t) & D (ddd) \\
7.31 & 6.85 \\
\hline
\end{tabular}

C ( $(\mathrm{t})$

$F(d d)$
4.14

E (d) G (s) H (s)

\begin{tabular}{l|l|l|}
\hline E (d) & $\mathrm{G}(\mathrm{s})$ & $\mathrm{H}(\mathrm{s})$ \\
4.24 & 3.82 & 2.95 \\
\hline
\end{tabular}

7.04
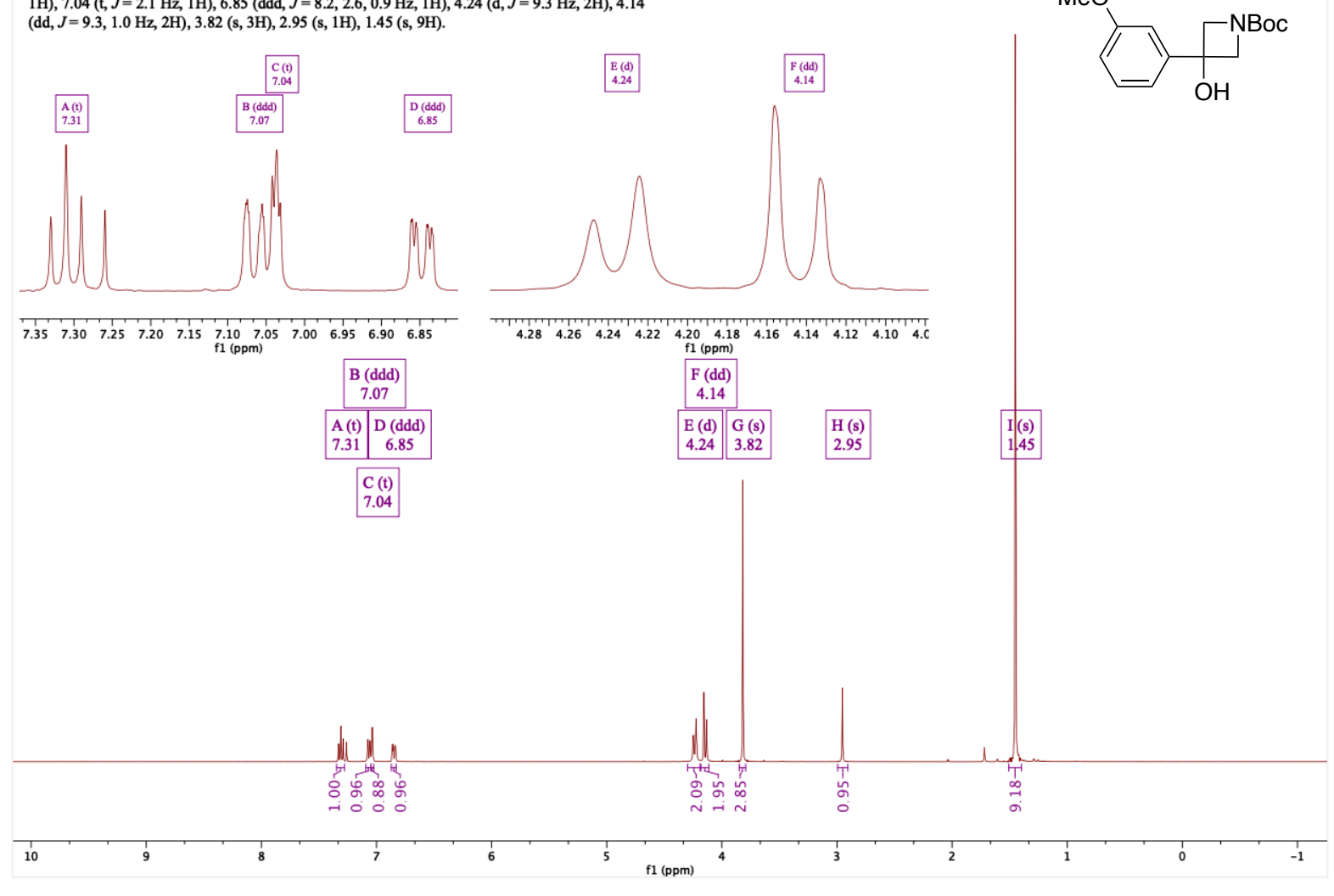

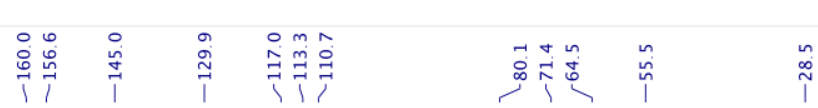

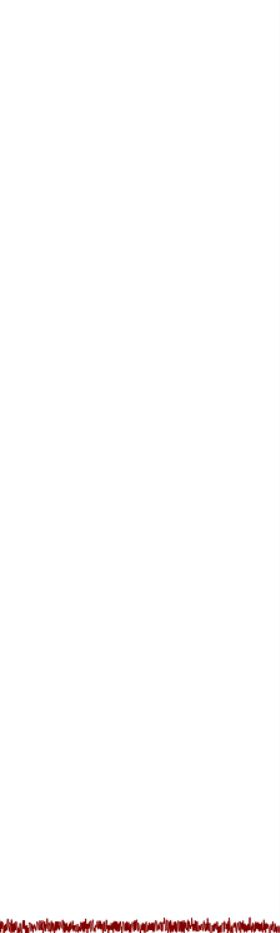

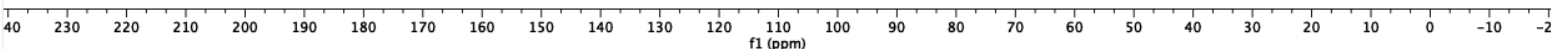


tert-Butyl 3-(3,4-dimethoxyphenyl)-3-hydroxyazetidine-1-carboxylate, 1i
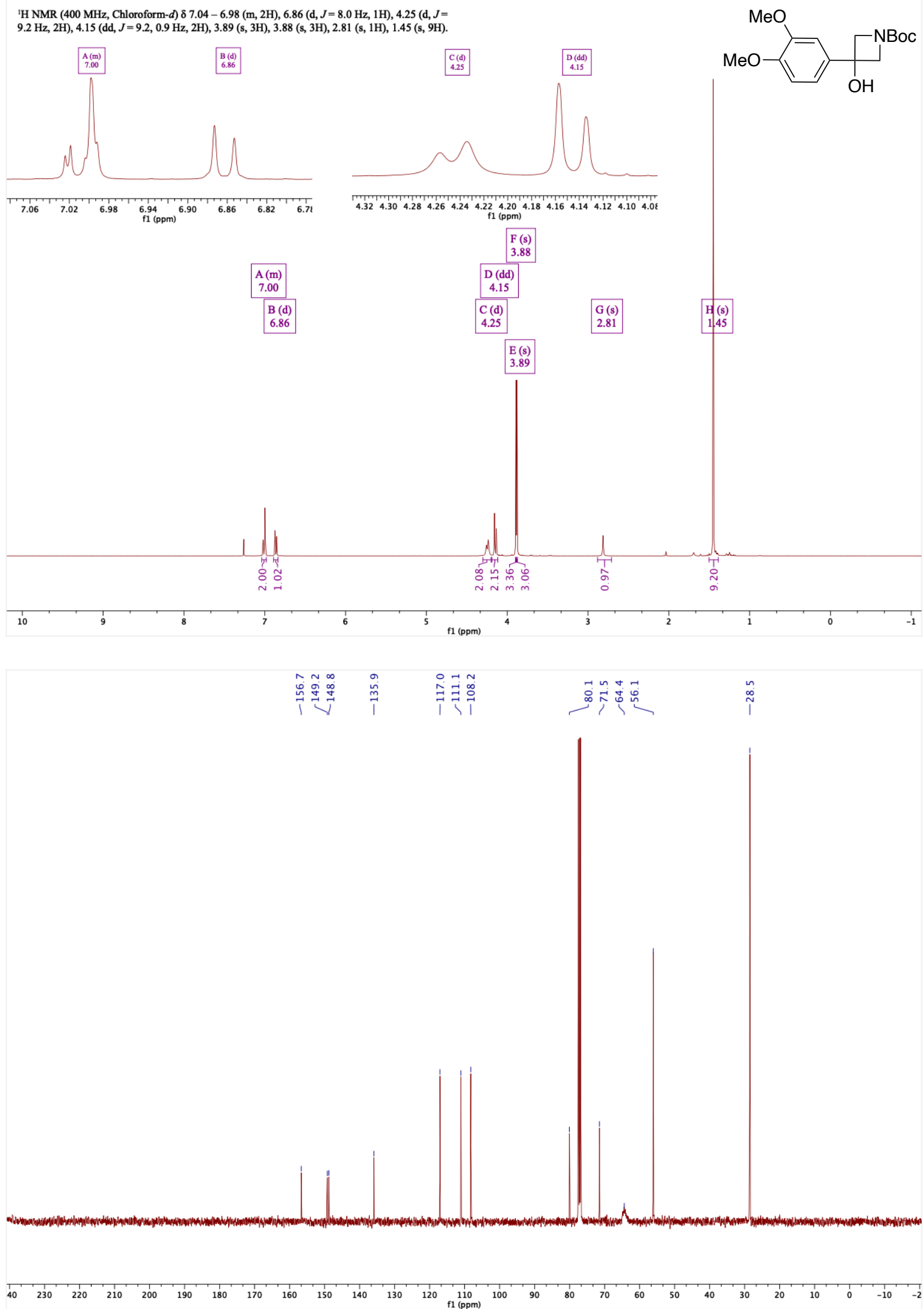
tert-Butyl 3-hydroxy-3-(2-methoxyphenyl)azetidine-1-carboxylate, $1 \mathrm{j}$

'H NMR ( $400 \mathrm{MHz}$, Chloroform- $d$ ) $\delta 7.34-7.24(\mathrm{~m}, 2 \mathrm{H}), 6.98(\mathrm{td}, J=7.5,1.0 \mathrm{~Hz}, 1 \mathrm{H}), 6.92$ (dd, $J=8.2,1.0 \mathrm{~Hz}, 1 \mathrm{H}), 4.34(\mathrm{~d}, J=9.5 \mathrm{~Hz}, 2 \mathrm{H}), 4.12(\mathrm{dd}, J=9.5,1.1 \mathrm{~Hz}, 2 \mathrm{H}), 3.88(\mathrm{~s}, 3 \mathrm{H})$, $3.42(\mathrm{~s}, 1 \mathrm{H}), 1.43(\mathrm{~s}, 9 \mathrm{H})$.
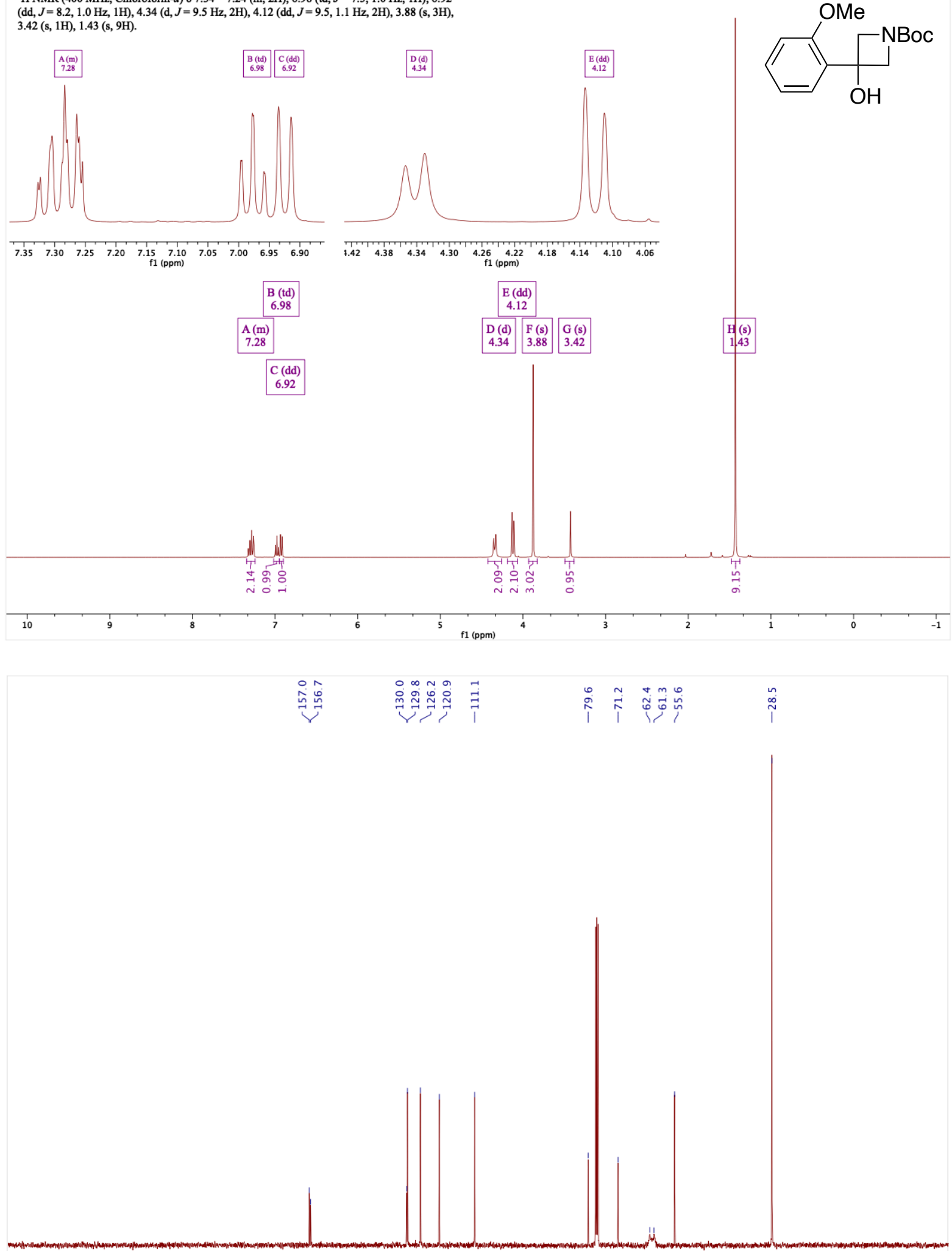

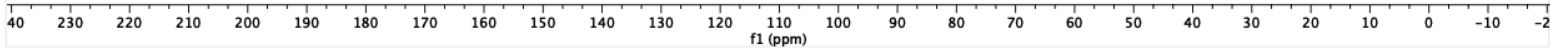


tert-Butyl 3-hydroxy-3-(3,4,5-trimethoxyphenyl)azetidine-1-carboxylate, $1 \mathrm{k}$
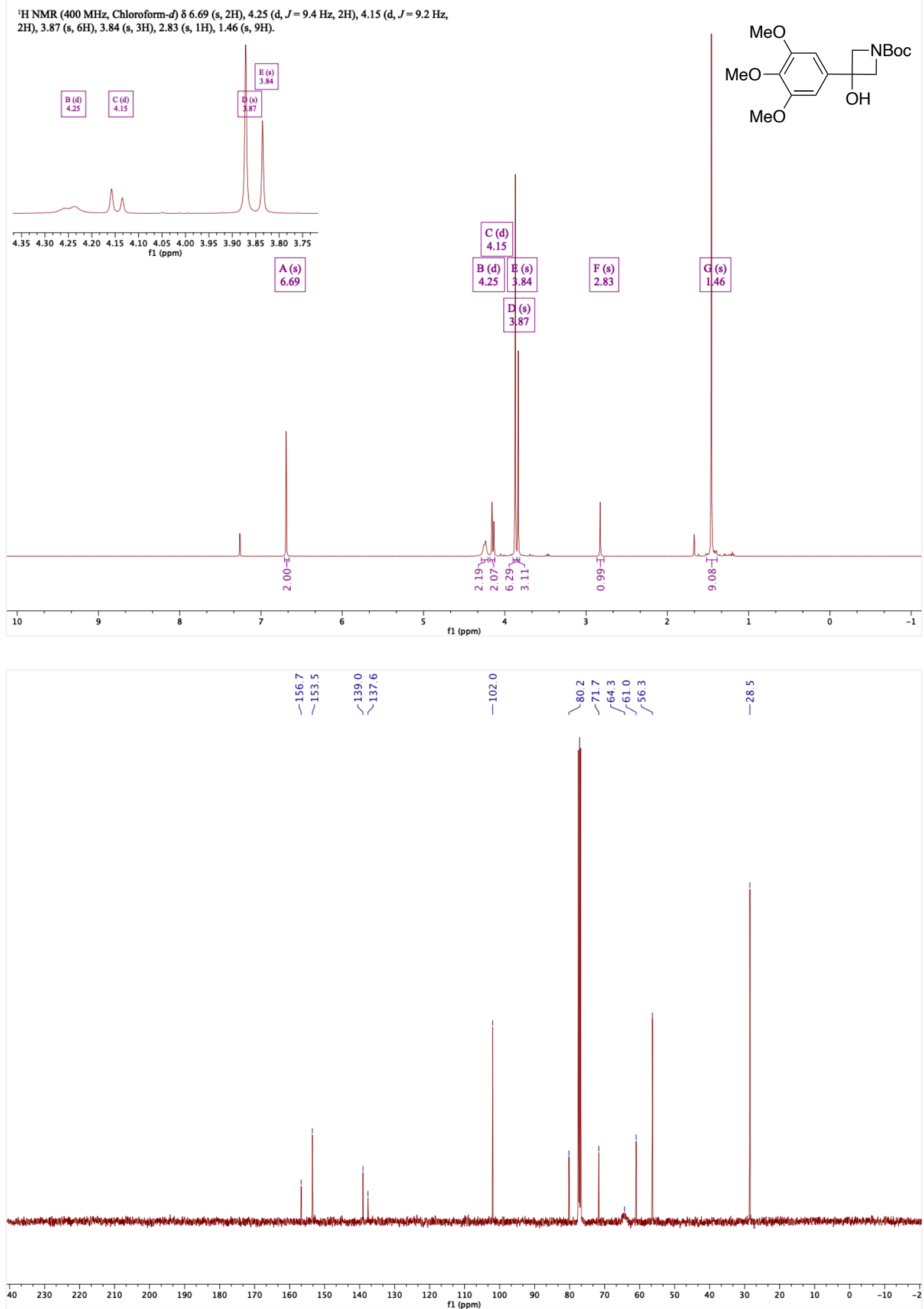
tert-Butyl 3-(4-chlorophenyl)-3-hydroxyazetidine-1-carboxylate, 11
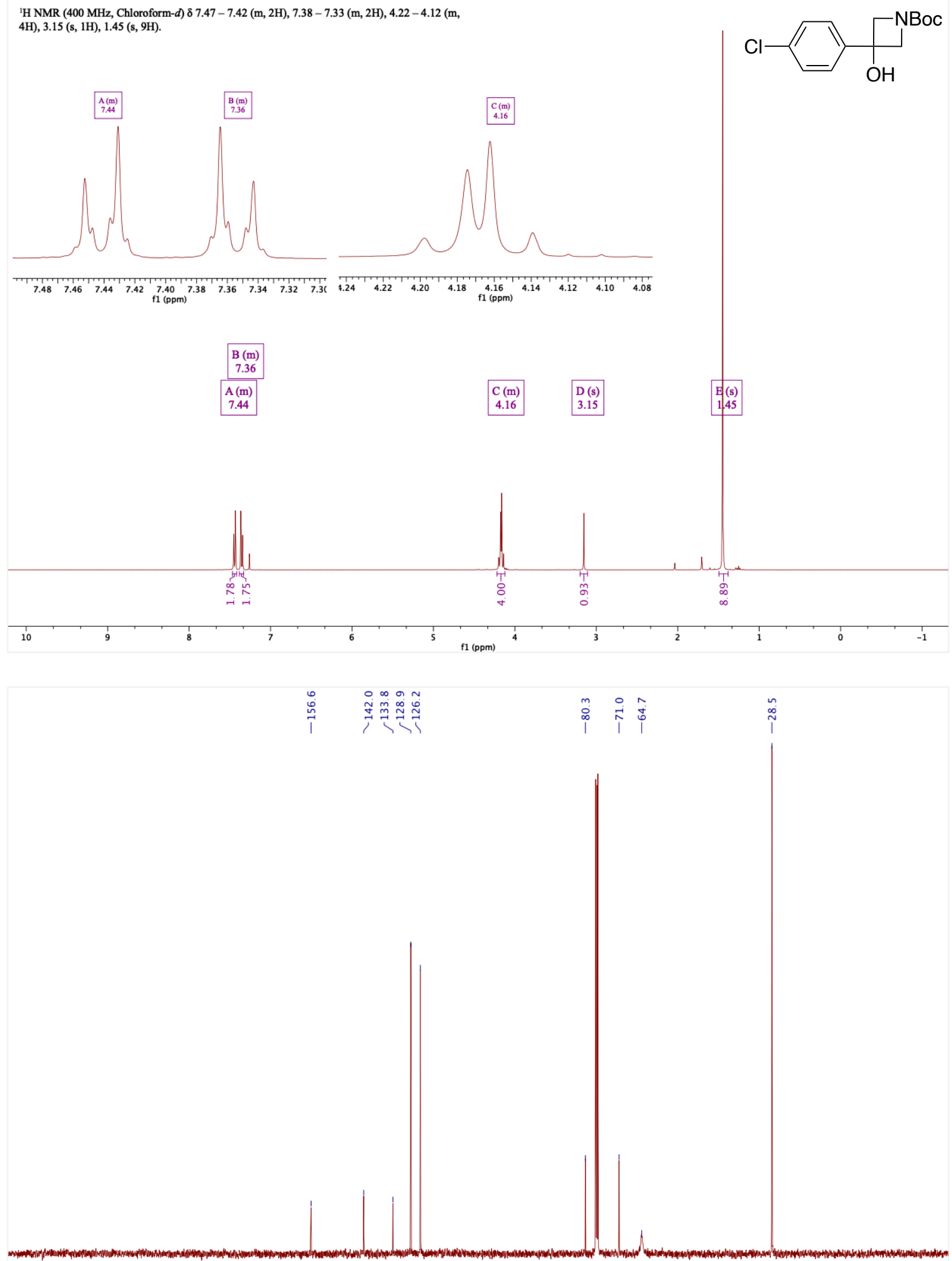

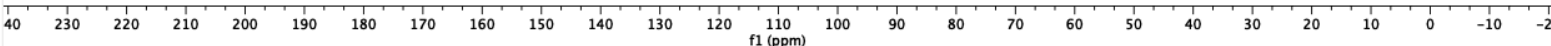


tert-Butyl 3-hydroxy-3-(4-(trifluoromethyl)phenyl)azetidine-1-carboxylate, $1 \mathrm{~m}$

${ }^{1} \mathrm{H}$ NMR (400 MHz, Chloroform- $\left.d\right) \delta 7.65(\mathrm{~s}, 4 \mathrm{H}), 4.25-4.16(\mathrm{~m}, 4 \mathrm{H}), 3.17$ (s, 1H), 1.46 (s, 9H)
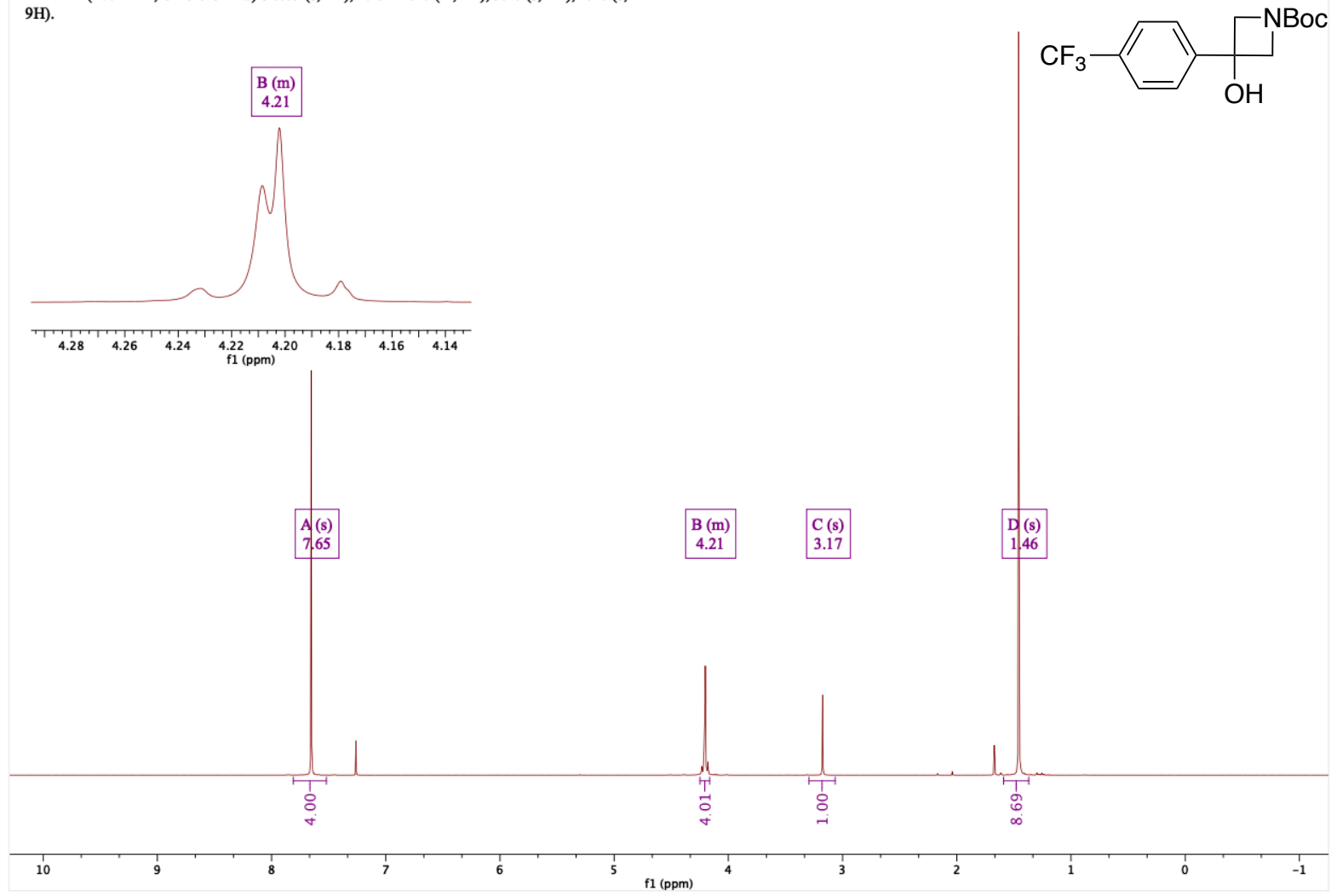

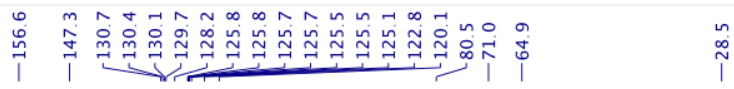

${ }^{13} \mathrm{C}$ NMR (101 MHz, Chloroform- $d$ ) $\delta 156.57,147.33,130.22$ (q, $\left.J=33.0 \mathrm{~Hz}\right), 125.73$ (q, $J=$ $3.9 \mathrm{~Hz}), 125.09,124.39(\mathrm{q}, J=271.8 \mathrm{~Hz}), 80.50,71.00,64.91,28.50$.

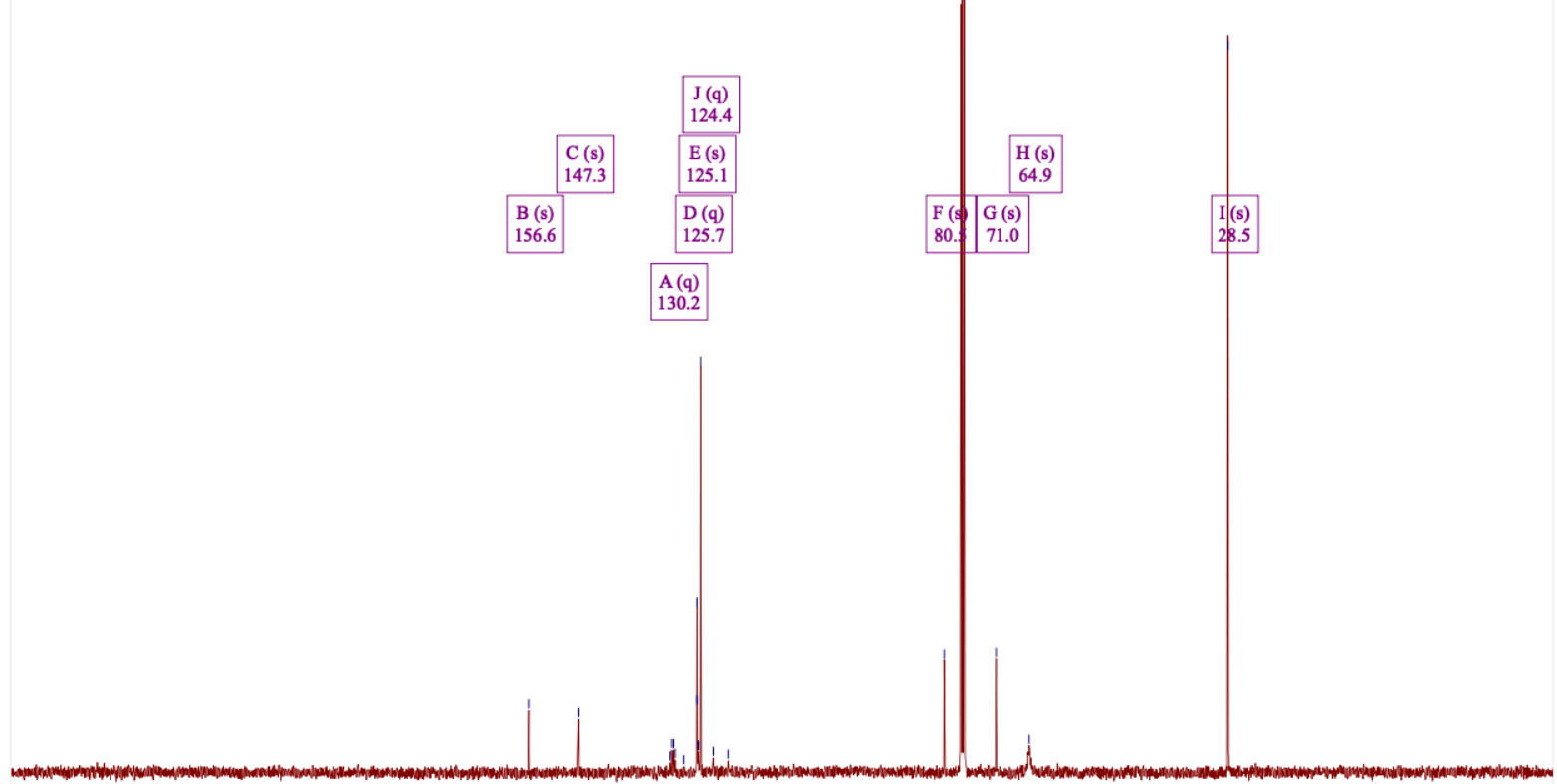

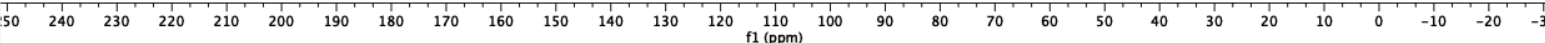



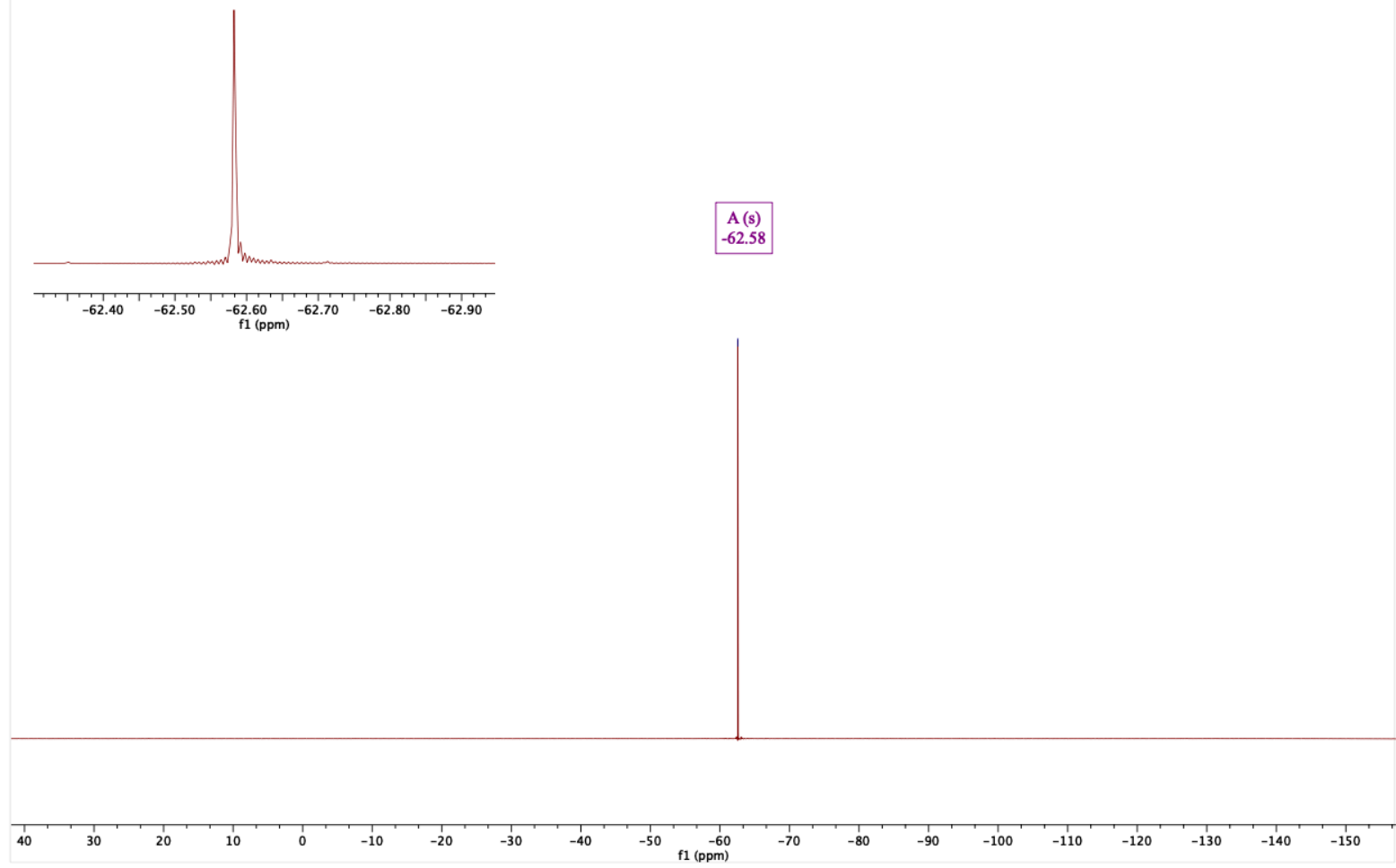
tert-Butyl 3-(benzo[b]thiophen-3-yl)-3-hydroxyazetidine-1-carboxylate, 1n

'H NMR ( $400 \mathrm{MHz}$, Chloroform- $d$ ) $87.90-7.85(\mathrm{~m}, 1 \mathrm{H}), 7.85-7.81(\mathrm{~m}, 1 \mathrm{H}), 7.41(\mathrm{~s}, 1 \mathrm{H})$ $7.41-7.37(\mathrm{~m}, 2 \mathrm{H}), 4.45(\mathrm{~d}, J=9.4 \mathrm{~Hz}, 2 \mathrm{H}), 4.28(\mathrm{~d}, J=9.3 \mathrm{~Hz}, 2 \mathrm{H}), 2.96(\mathrm{~s}, 1 \mathrm{H}), 1.44(\mathrm{~s}$, $9.41-7$.

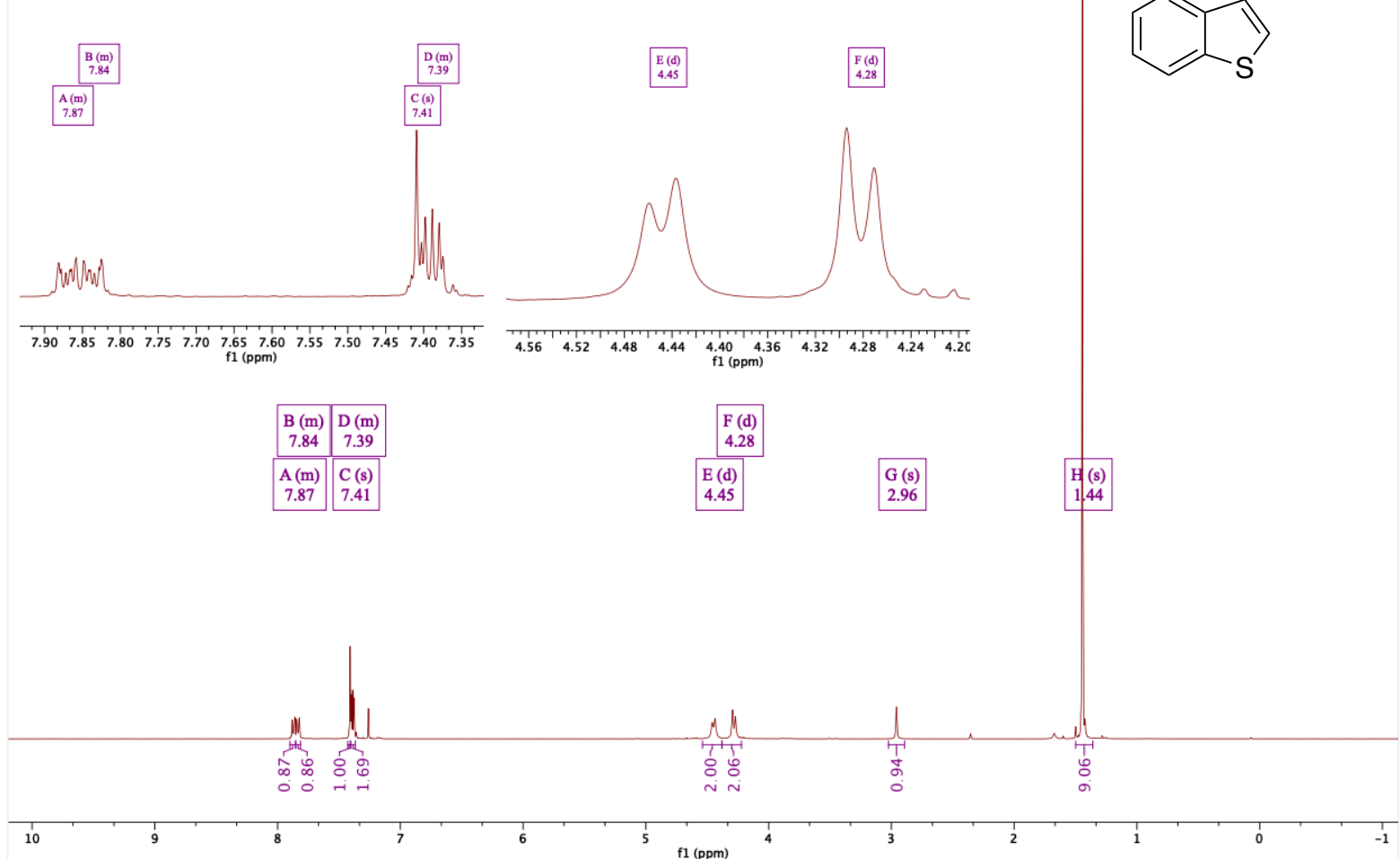<smiles>O=C(O)[Nb]1CC(O)(c2csc3ccccc23)C1</smiles>

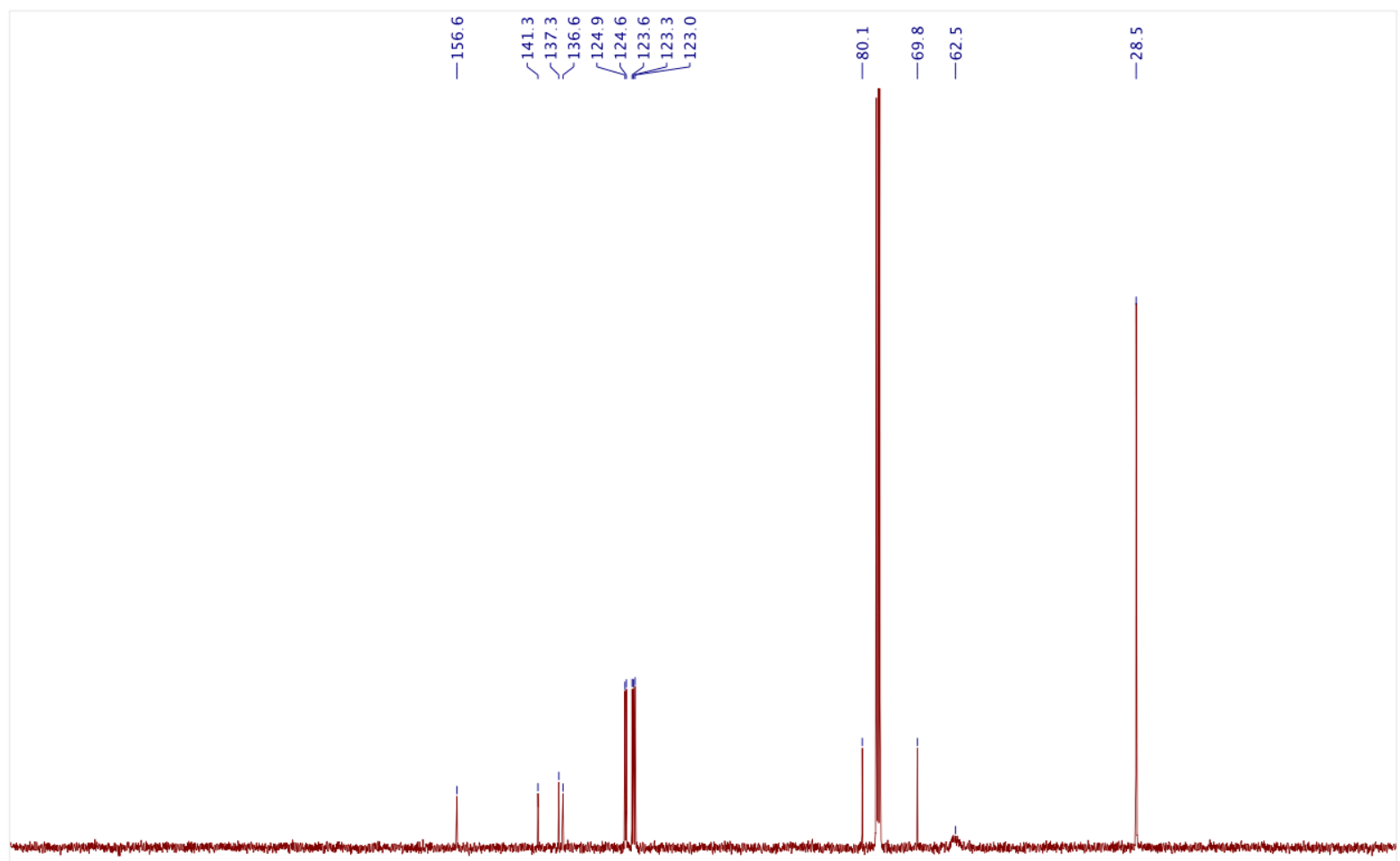

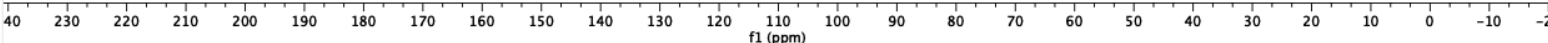


tert-Butyl 3-hydroxy-3-(thiophen-2-yl)azetidine-1-carboxylate, 10

${ }^{1} \mathrm{H}$ NMR (400 MHz, Chloroform- $d$ ) $\delta 7.28$ (dd, $J=5.1,1.2 \mathrm{~Hz}, 1 \mathrm{H}$ ), 7.09 (dd, $J=3.6,1.2 \mathrm{~Hz}$ $1 \mathrm{H}), 7.00$ (dd, $J=5.1,3.6 \mathrm{~Hz}, 1 \mathrm{H}), 4.25$ (d, $J=9.3 \mathrm{~Hz}, 2 \mathrm{H}), 4.19$ (dd, $J=9.3,1.0 \mathrm{~Hz}, 2 \mathrm{H}), 3.14$ $(\mathrm{s}, 1 \mathrm{H}), 1.45(\mathrm{~s}, 9 \mathrm{H})$.
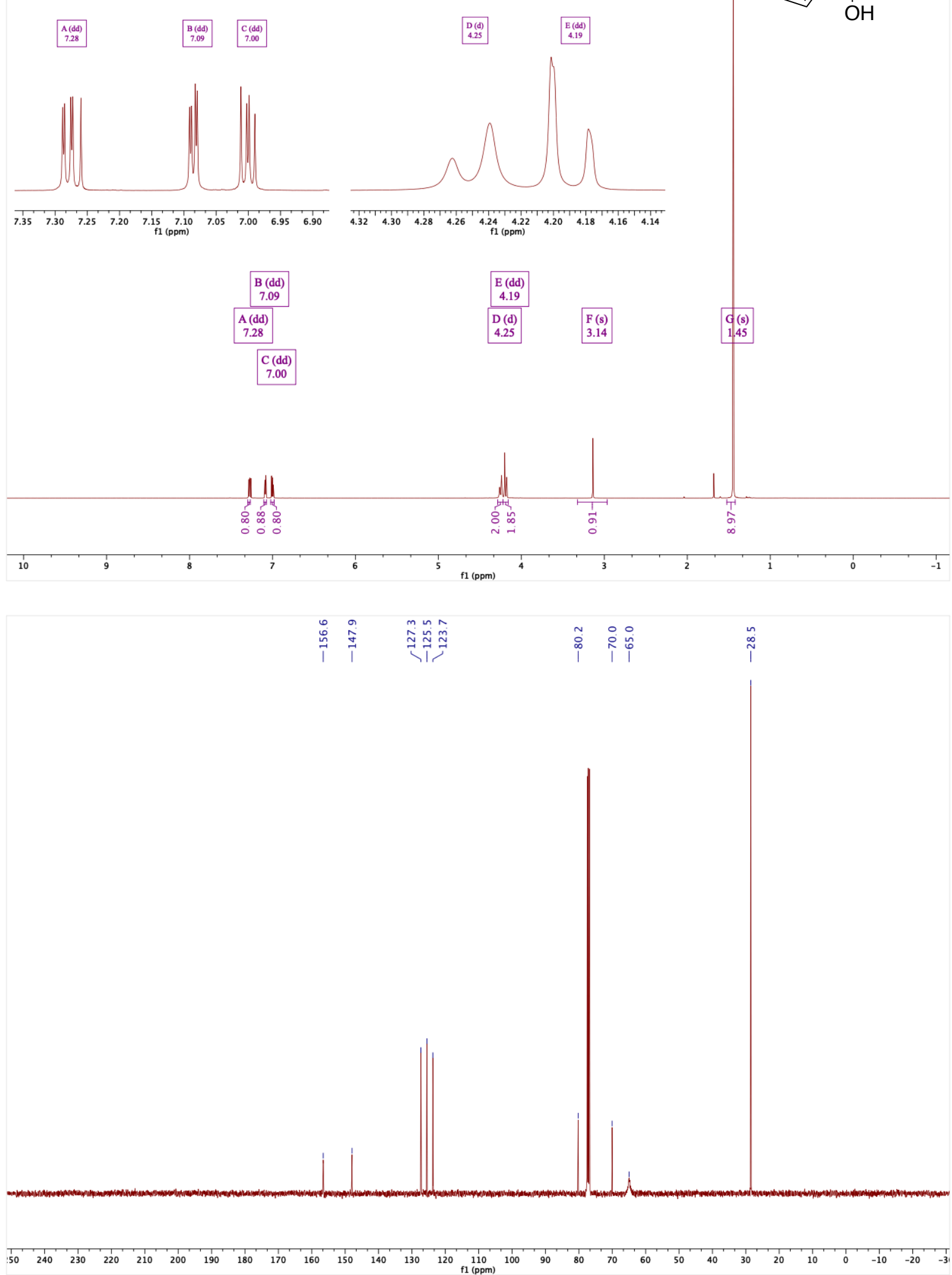
tert-Butyl 3-hydroxy-3-(1-tosyl-1H-indol-2-yl)azetidine-1-carboxylate, 1p

${ }^{1} \mathrm{H}$ NMR (400 MHz, Chloroform- $\left.d\right) \delta 7.87-7.80(\mathrm{~m}, 1 \mathrm{H}), 7.68-7.61(\mathrm{~m}, 2 \mathrm{H}), 7.54-7.48(\mathrm{~m}$, $4.27(\mathrm{dd}, J=9.6,1.0 \mathrm{~Hz}, 2 \mathrm{H}), 2.33(\mathrm{~s}, 3 \mathrm{H}), 1.44(\mathrm{~s}, 9 \mathrm{H})$
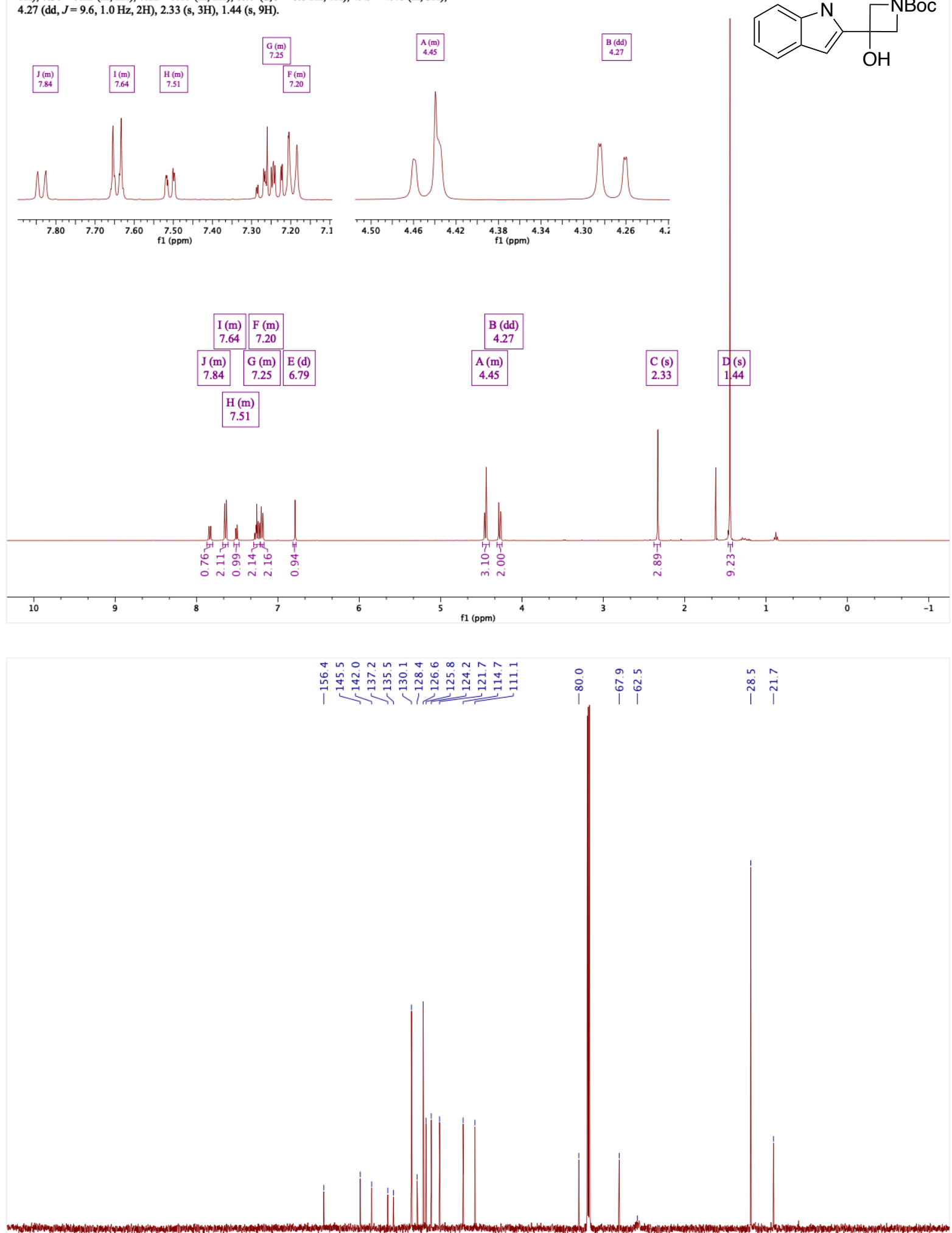

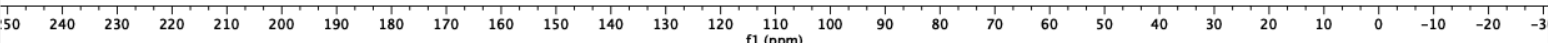


tert-Butyl 3-(benzofuran-2-yl)-3-hydroxyazetidine-1-carboxylate, 1q

${ }^{1} \mathrm{H}$ NMR ( $400 \mathrm{MHz}$, Chloroform- $d$ ) 87.60 - 7.54 (m, 1H), $7.52-7.47$ (m, 1H), 7.32 (ddd, $J=$ $8.2,7.2,1.5 \mathrm{~Hz}, 1 \mathrm{H}), 7.26(\mathrm{td}, J=7.4,1.1 \mathrm{~Hz}, 1 \mathrm{H}), 6.75(\mathrm{~d}, J=1.0 \mathrm{~Hz}, 1 \mathrm{H}), 4.41(\mathrm{~d}, J=9.3 \mathrm{~Hz}$ $2 \mathrm{H}), 4.19(\mathrm{dd}, J=9.3,1.0 \mathrm{~Hz}, 2 \mathrm{H}), 3.73(\mathrm{~s}, 1 \mathrm{H}), 1.48(\mathrm{~s}, 9 \mathrm{H})$.
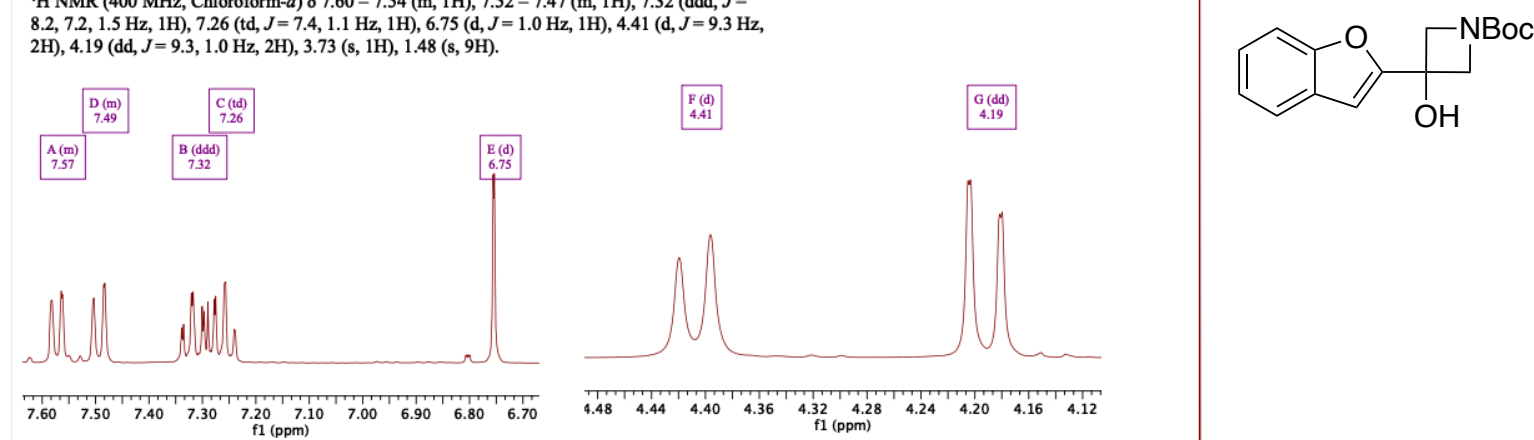

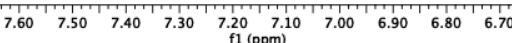
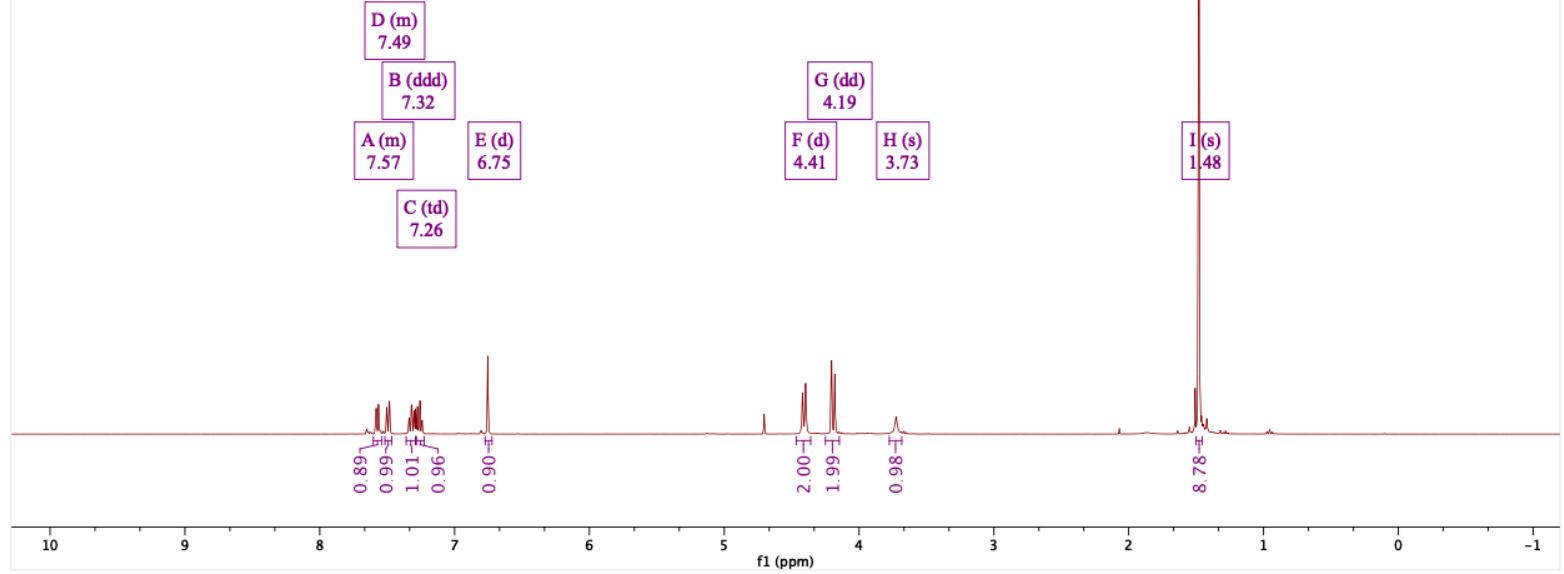

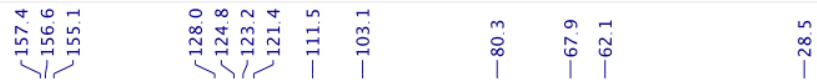

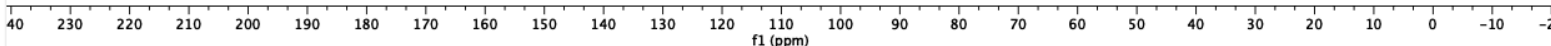


tert-Butyl (2-(benzo[b]thiophen-2-yl)-2-oxoethyl)carbamate, 2a
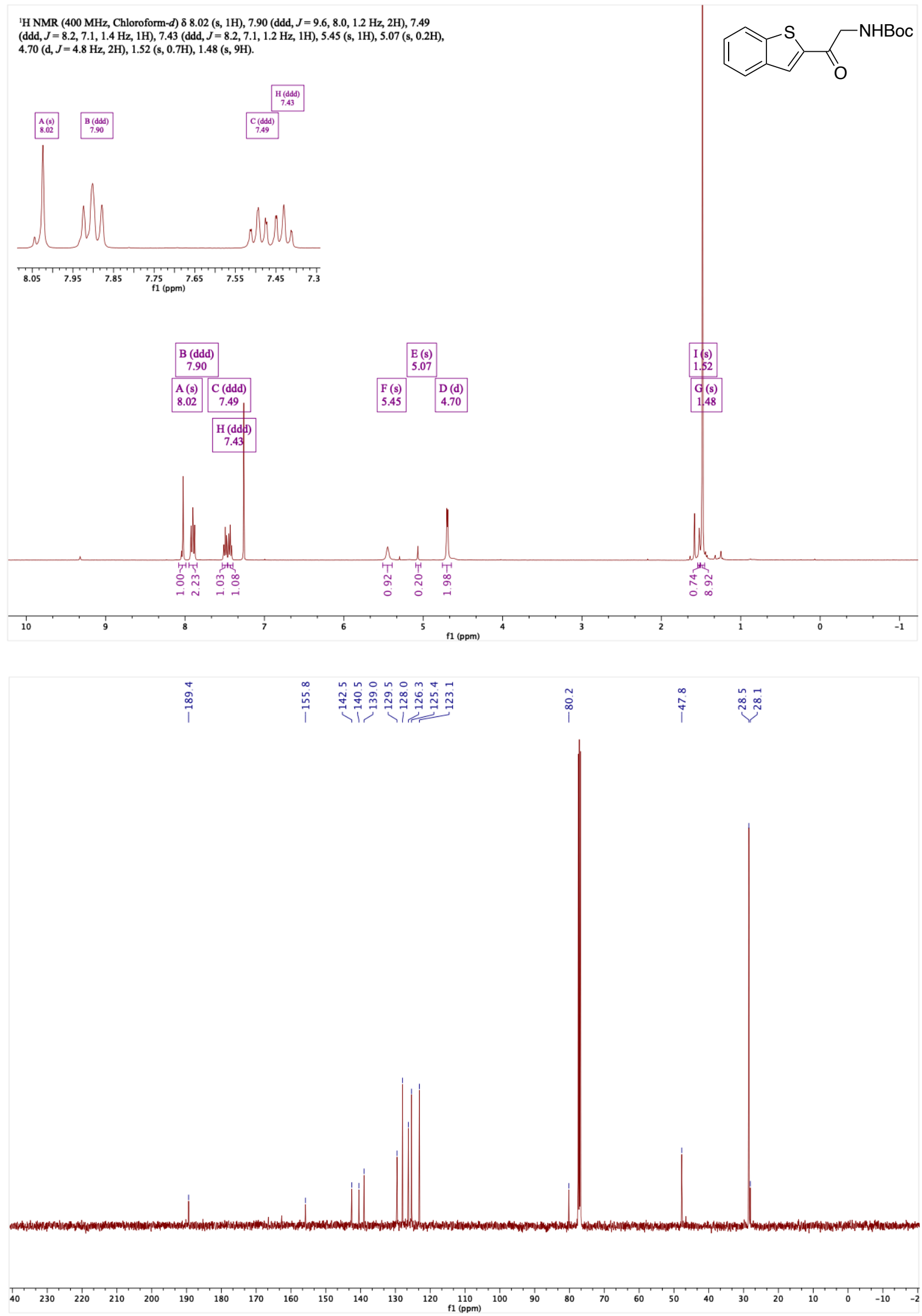


\section{$N$-(2-(Benzo[b]thiophen-2-yl)-2-oxoethyl)-4-methylbenzenesulfonamide}
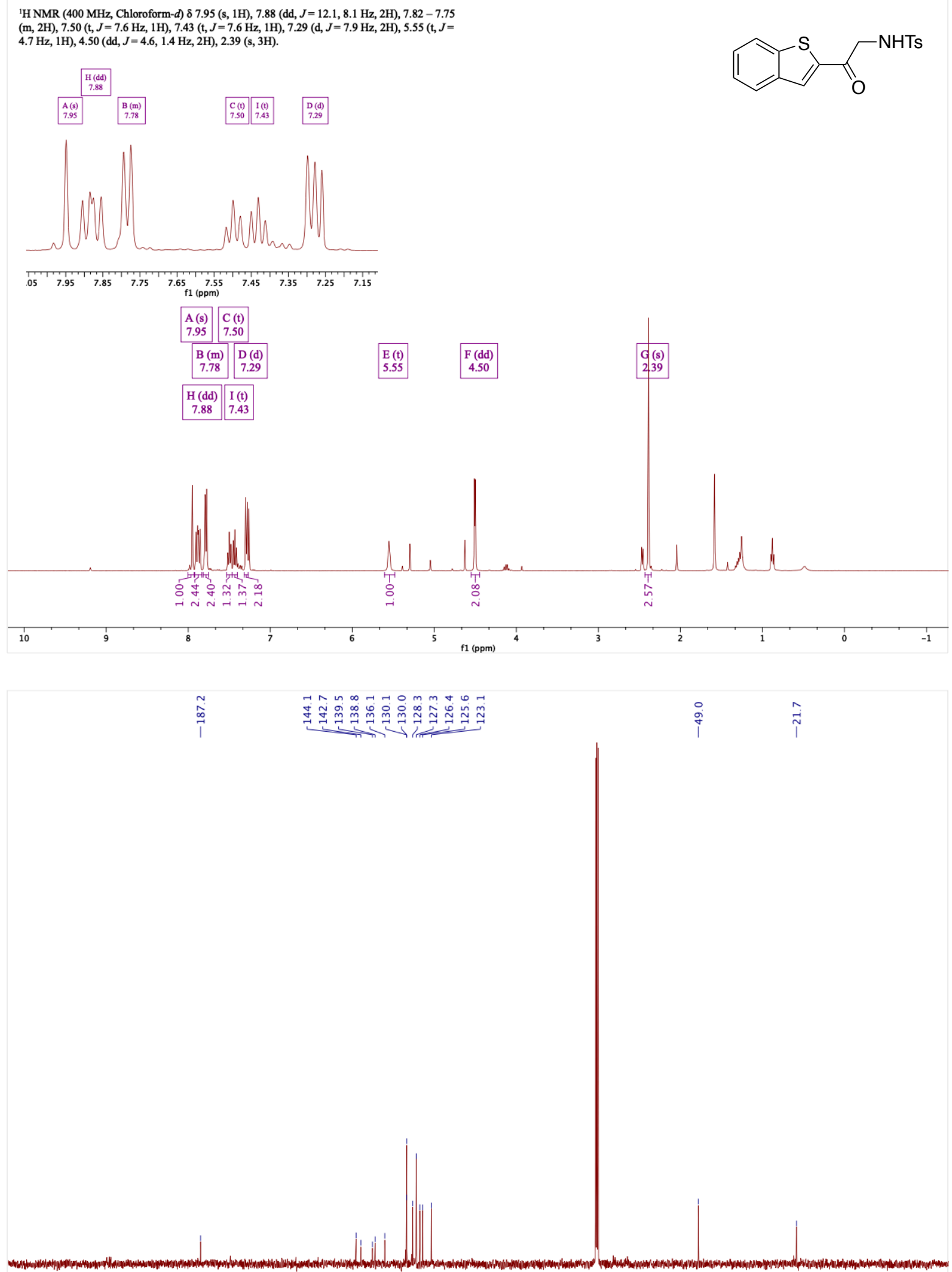

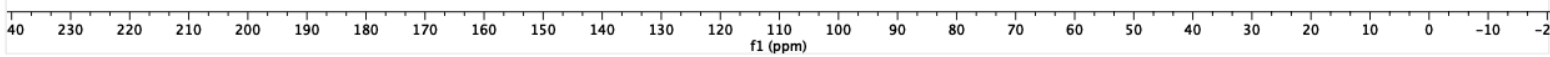


tert-Butyl (2-oxo-2-phenylethyl)carbamate, 2b
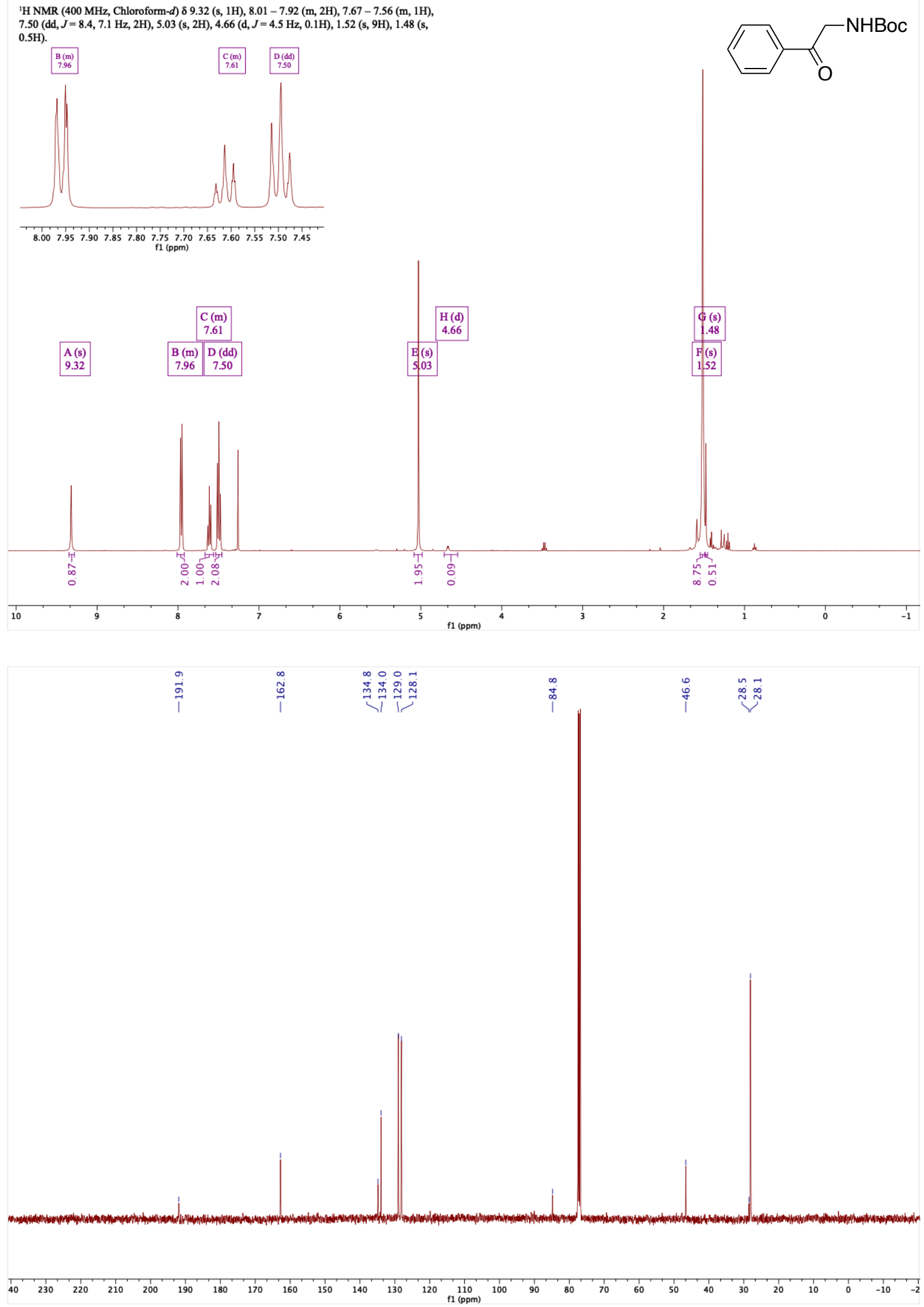
tert-Butyl (2-(4-fluorophenyl)-2-oxoethyl)carbamate, 2c

${ }^{1} \mathrm{H}$ NMR $(400 \mathrm{MHz}$, Chloroform- $d$ ) $\delta 9.30(\mathrm{~s}, 1 \mathrm{H}), 8.08-7.92(\mathrm{~m}, 2 \mathrm{H}), 7.22-7.11(\mathrm{~m}, 2 \mathrm{H})$ $4.99(\mathrm{~s}, 2 \mathrm{H}), 4.62(\mathrm{~d}, J=4.6 \mathrm{~Hz}, 0.1 \mathrm{H}), 1.51(\mathrm{~s}, 9 \mathrm{H}), 1.47(\mathrm{~s}, 0.7 \mathrm{H})$.

\begin{tabular}{|l|}
\hline A(m) \\
7.99 \\
\hline
\end{tabular}

\begin{tabular}{|c|}
\hline B (m) \\
7.17 \\
\hline
\end{tabular}

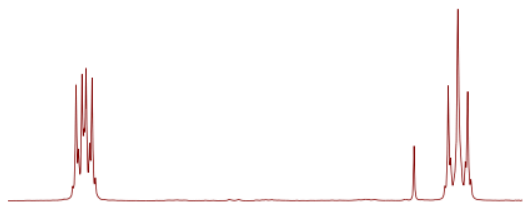

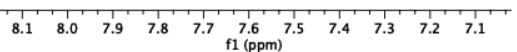
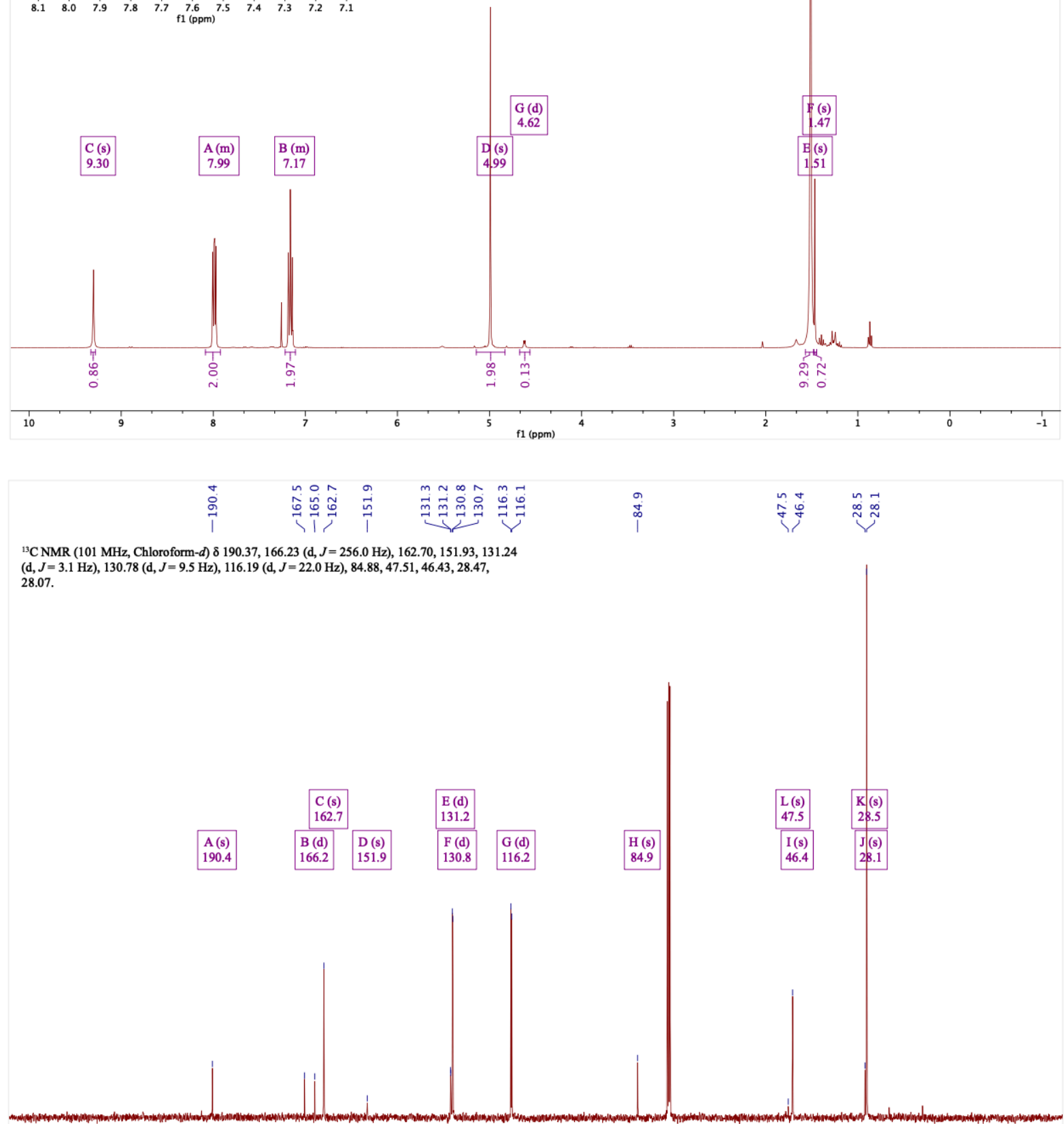

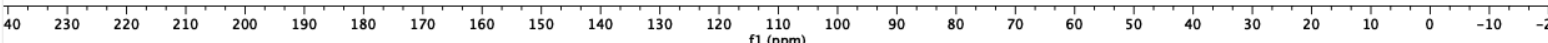




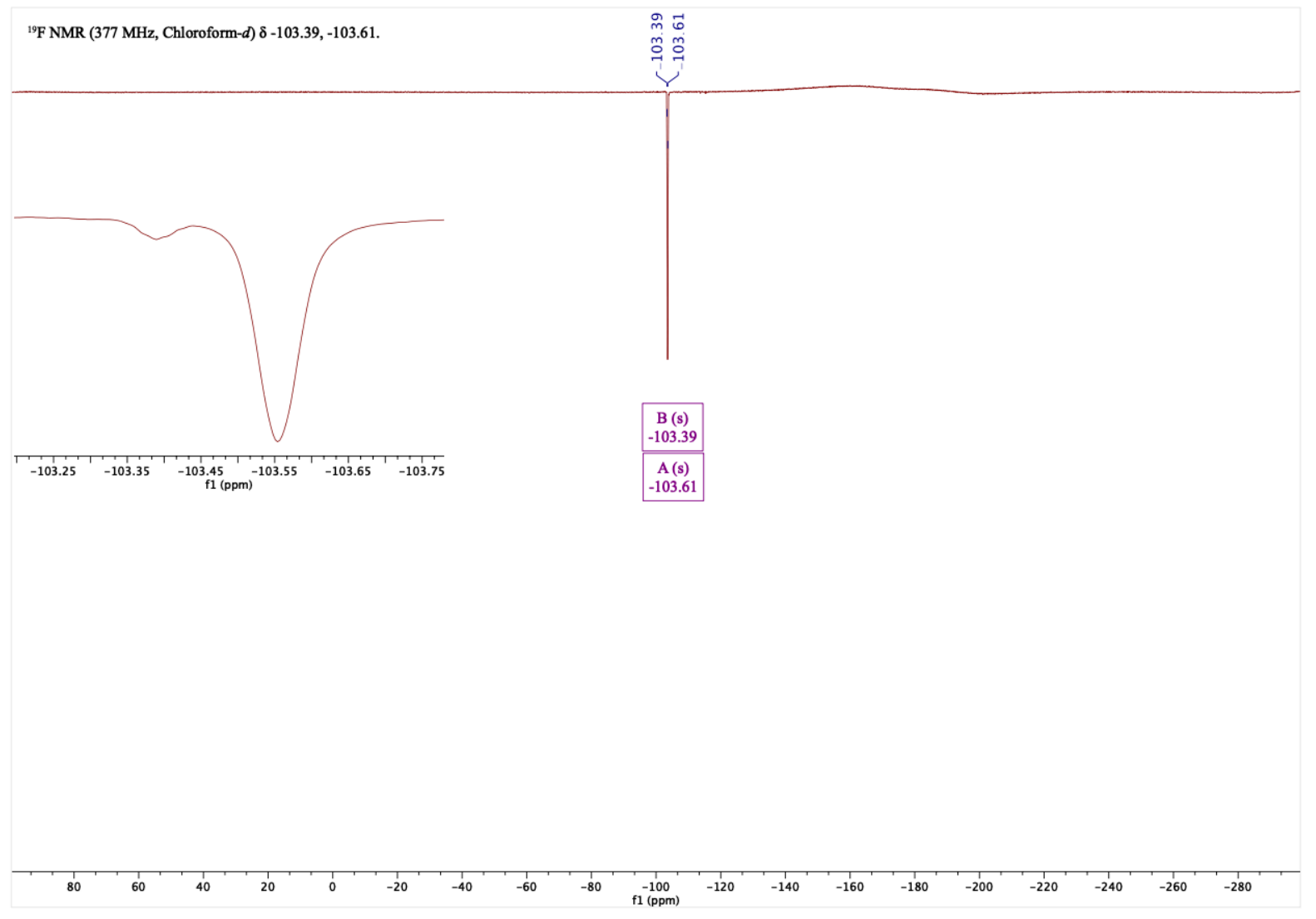


tert-Butyl (2-(3-fluorophenyl)-2-oxoethyl)carbamate, 2d

${ }^{1} \mathrm{H}$ NMR $(400 \mathrm{MHz}$, Chloroform- $d$ ) $\delta 9.29(\mathrm{~s}, 1 \mathrm{H}), 7.73$ (dt, $J=7.7,1.3 \mathrm{~Hz}, 1 \mathrm{H}$ ), 7.63 (ddd, $J=$ $9.2,2.6,1.5 \mathrm{~Hz}, 1 \mathrm{H}), 7.48(\mathrm{td}, J=8.0,5.4 \mathrm{~Hz}, 1 \mathrm{H}), 7.31$ (tdd, $J=8.3,2.6,1.0 \mathrm{~Hz}, 1 \mathrm{H}), 4.99(\mathrm{~s}$,

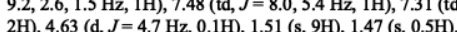

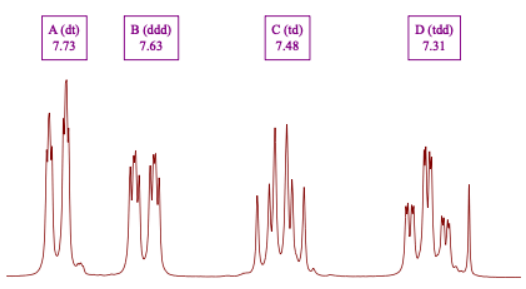

$\begin{array}{lllllllllllll}7.75 & 7.70 & 7.65 & 7.60 & 7.55 & 7.50 & 7.45 & 7.40 & 7.35 & 7.30 & 7.25\end{array}$
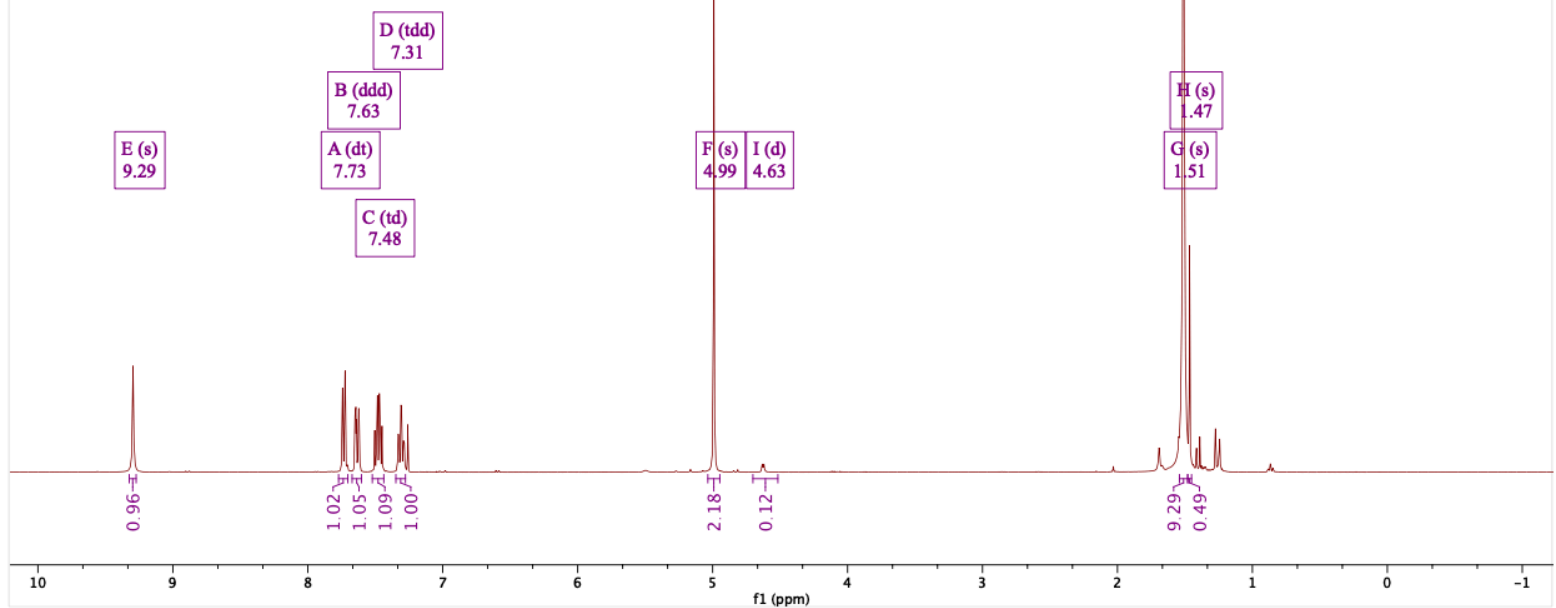

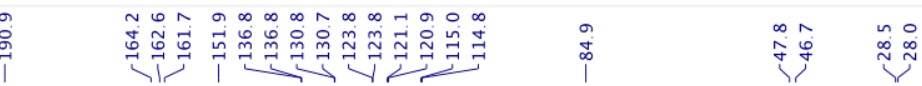

${ }^{13} \mathrm{CNMR}$ (101 MHz, Chloroform- $d$ ) $\delta$ 190.87, 162.98 (d, $J=248.8 \mathrm{~Hz}$ ), 162.63, 151.88, 136.80 $(\mathrm{d}, J=6.3 \mathrm{~Hz}), 130.73(\mathrm{~d}, J=7.8 \mathrm{~Hz}), 123.81(\mathrm{~d}, J=3.1 \mathrm{~Hz}), 121.02(\mathrm{~d}, J=21.3 \mathrm{~Hz}), 114.91$ (d, $J=22.4 \mathrm{~Hz}$ ), 84.93, 47.80, 46.66, 28.46, 28.05.
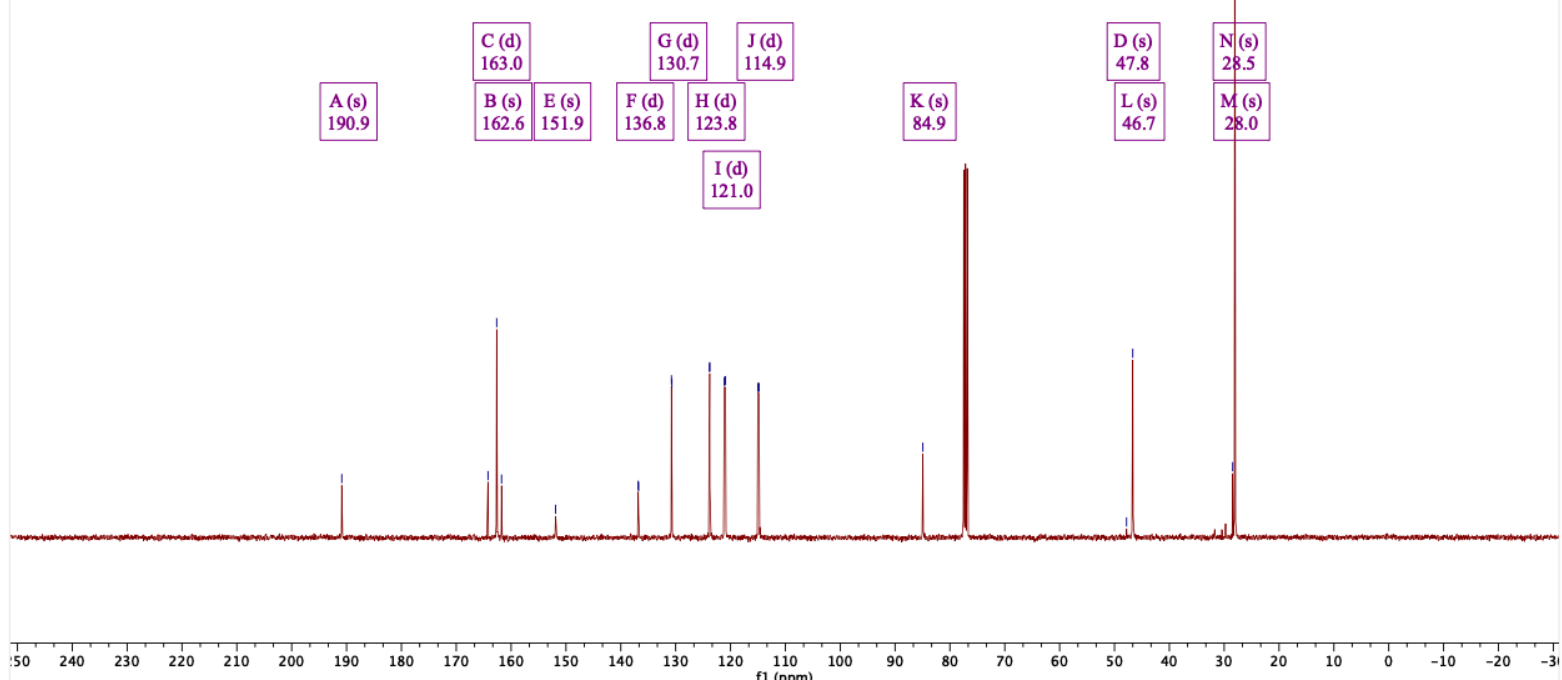
${ }^{19} \mathrm{~F}$ NMR (377 MHz, Chloroform- $d$ ) 8 -111.07.

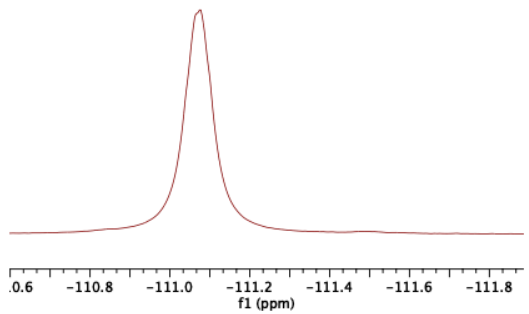




\section{tert-Butyl (2-(2-fluorophenyl)-2-oxoethyl)carbamate, 2e}

${ }^{1} \mathrm{H}$ NMR ( $400 \mathrm{MHz}$, Chloroform- $d$ ) $89.29(\mathrm{~s}, 1 \mathrm{H}), 8.00-7.86(\mathrm{~m}, 1 \mathrm{H}), 7.57$ (dddd, $J=8.4,7.1$, $5.1,1.9 \mathrm{~Hz}, 1 \mathrm{H}), 7.25(\mathrm{td}, J=7.5,1.1 \mathrm{~Hz}, 1 \mathrm{H}), 7.17$ (ddd, $J=11.3,8.4,1.1 \mathrm{~Hz}, 1 \mathrm{H}), 4.95$ (d, $J$ $=3.5 \mathrm{~Hz}, 2 \mathrm{H}), 4.58(\mathrm{dd}, J=5.0,3.2 \mathrm{~Hz}, 0 \mathrm{H}), 1.51(\mathrm{~s}, 9 \mathrm{H}), 1.46(\mathrm{~s}, 1 \mathrm{H})$.
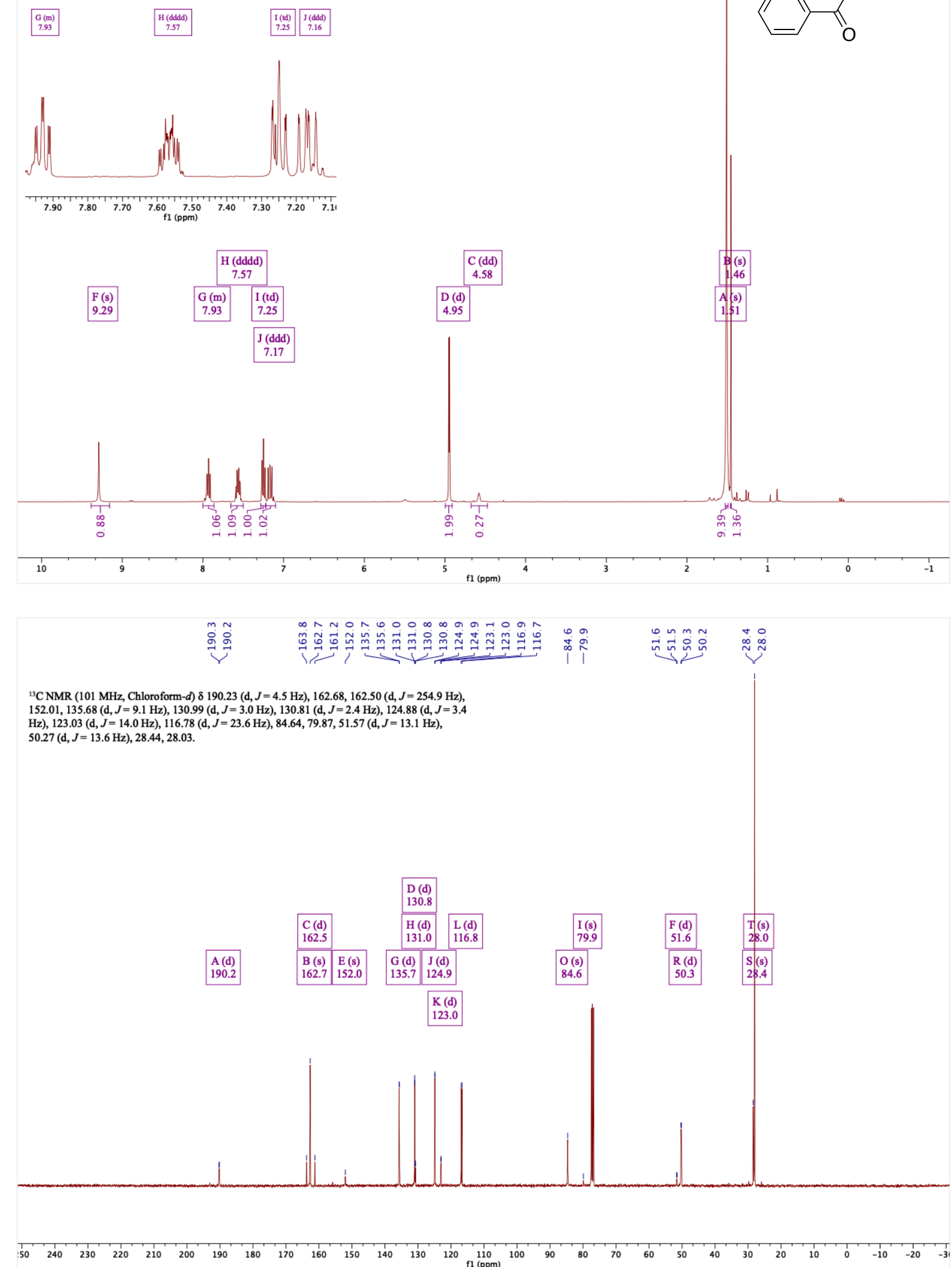
${ }^{19} \mathrm{~F}$ NMR (377 MHz, Chloroform- $d$ ) $\delta$-107.66, -107.80.

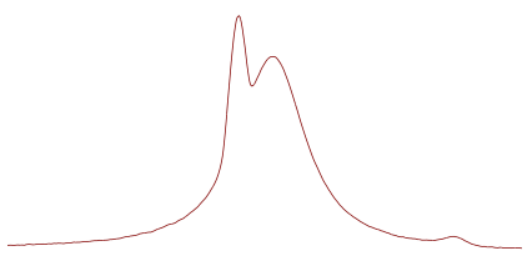

$-107.0-107.2-107.4-107.6-107.8-108.0-108.2-108.4-108.6$
:

옹

\begin{tabular}{c}
$\mathrm{B}(\mathrm{s})$ \\
-107.66 \\
\hline
\end{tabular}

\begin{tabular}{c}
\hline $\mathbf{A}(\mathbf{s})$ \\
\hline-107.80
\end{tabular}

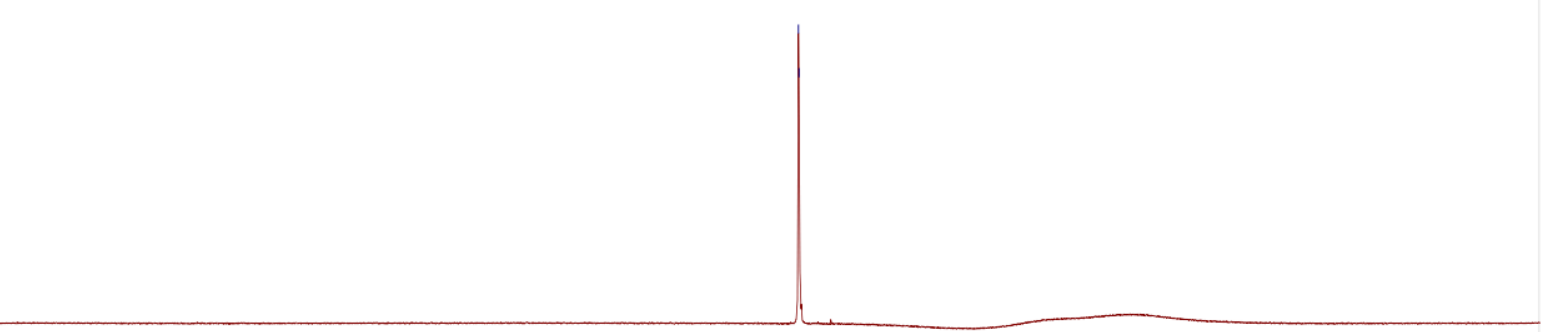

$80 \quad \frac{1}{6}+\frac{1}{40}$

$20+0$

$-20 \quad-40$

$-60$

$-80 \quad-100$

$-120$

$-180+-200$

$-220$

$-260$

$-280$ 
tert-Butyl (2-(2,6-difluorophenyl)-2-oxoethyl)carbamate, $2 \mathrm{f}$

${ }^{1} \mathrm{H}$ NMR ( $400 \mathrm{MHz}$, Chloroform- $d$ ) $\delta 9.28$ (s, 1H), 7.47 (tt, $\left.J=8.4,6.2 \mathrm{~Hz}, 1 \mathrm{H}\right), 7.03-6.95$ (m, $2 \mathrm{H}), 4.84(\mathrm{t}, J=1.3 \mathrm{~Hz}, 2 \mathrm{H}), 1.51(\mathrm{~s}, 9 \mathrm{H})$
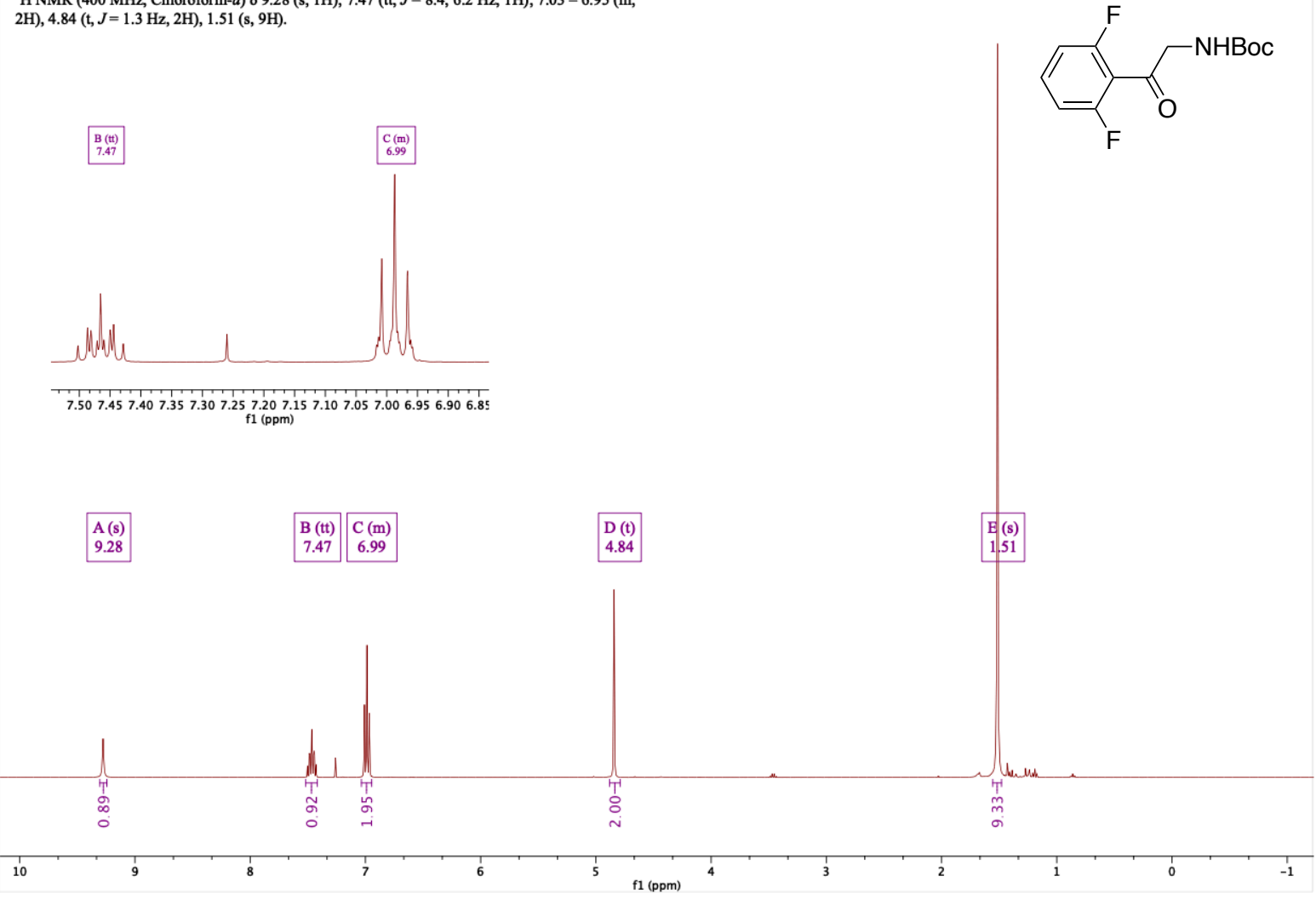

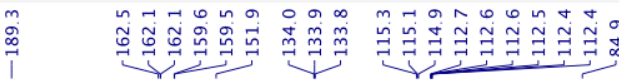

${ }^{13} \mathrm{C}$ NMR (101 MHz, Chloroform- $d$ ) $\delta 189.33,162.50,160.83$ (dd, $\left.J=256.5,6.9 \mathrm{~Hz}\right), 151.92$ $133.92(\mathrm{t}, J=10.8 \mathrm{~Hz}), 115.09(\mathrm{t}, J=18.1 \mathrm{~Hz}), 112.78-112.30(\mathrm{~m}), 84.94,50.72(\mathrm{~d}, J=5.6$ $\mathrm{Hz}), 27.96$

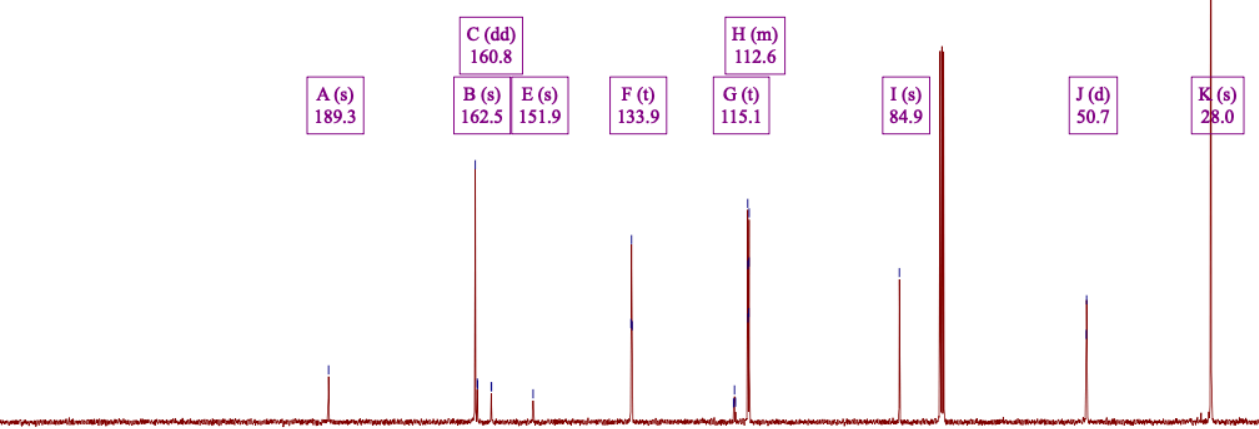

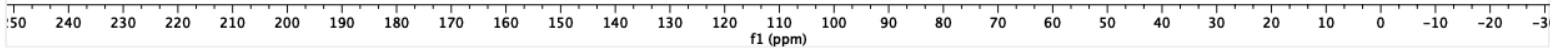


${ }^{19} \mathrm{~F}$ NMR (377 MHz, Chloroform- $\left.d\right) \delta-110.20,-110.62$.

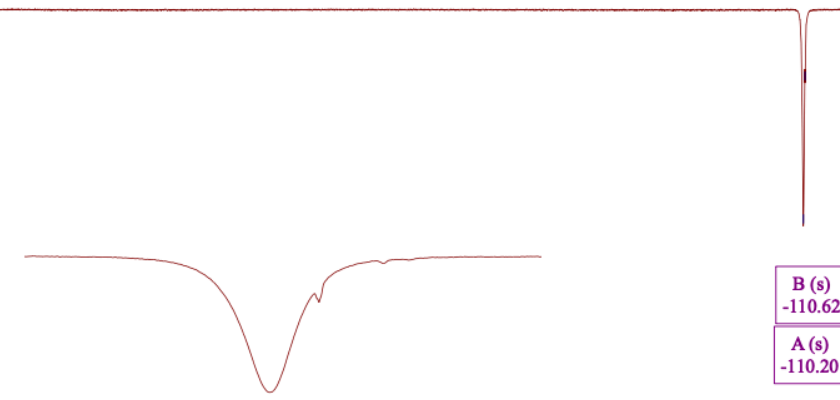

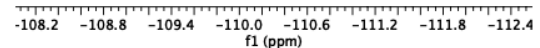

\begin{tabular}{l}
$\mathrm{B}(\mathrm{s})$ \\
110.62 \\
$\mathrm{~A}(\mathrm{~s})$ \\
\hline 110.20
\end{tabular}

110.20

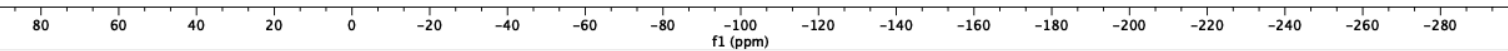


tert-Butyl (2-(4-methoxyphenyl)-2-oxoethyl)carbamate, 2g

${ }^{1} \mathrm{H}$ NMR $(400 \mathrm{MHz}$, Chloroform- $d$ ) $\delta 9.31$ (s, 0.3H), $8.10-7.76(\mathrm{~m}, 2 \mathrm{H}), 7.09-6.69(\mathrm{~m}, 2 \mathrm{H})$,

$5.57(\mathrm{~s}, 0.6 \mathrm{H}), 4.98(\mathrm{~s}, 0.8 \mathrm{H}), 4.60(\mathrm{~d}, J=4.5 \mathrm{~Hz}, 1.3 \mathrm{H}), 3.87(\mathrm{~d}, J=1.4 \mathrm{~Hz}, 3 \mathrm{H}), 1.50(\mathrm{~s}$,

$3.57(\mathrm{~s}, 0.6 \mathrm{H}), 1.47(\mathrm{~s}, 5.5 \mathrm{H})$

$\mathrm{MeO}=\mathrm{C}_{\mathrm{O}}^{\mathrm{NHBoc}}$

$\left[\begin{array}{l}8(0,1) \\ 7,33\end{array}\right.$

\begin{tabular}{c}
$\substack{c(m) \\
6.95}$ \\
\hline
\end{tabular}

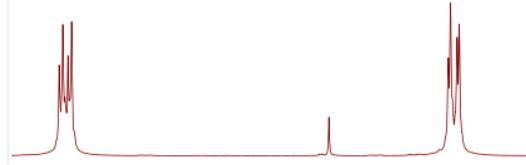

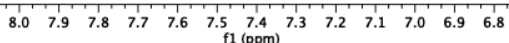

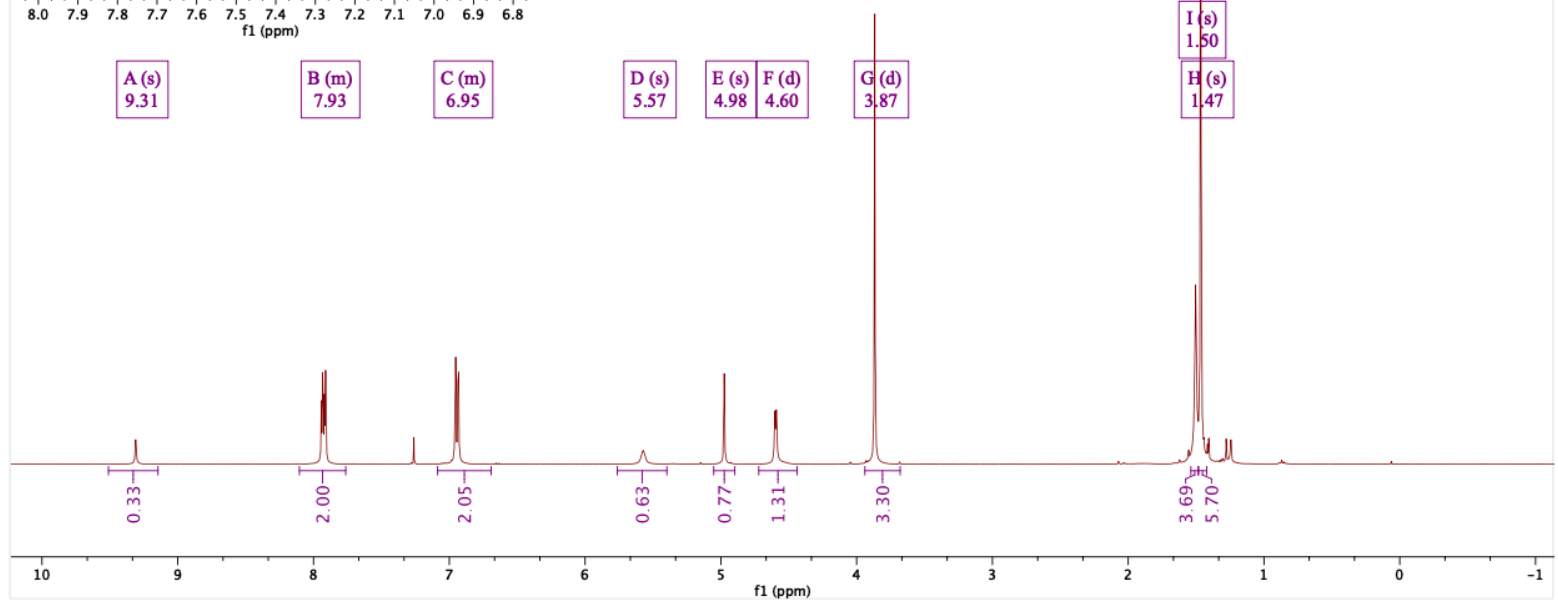

占酋

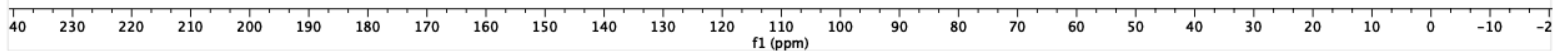


tert-Butyl (2-(3-methoxyphenyl)-2-oxoethyl)carbamate, $2 \mathrm{~h}$
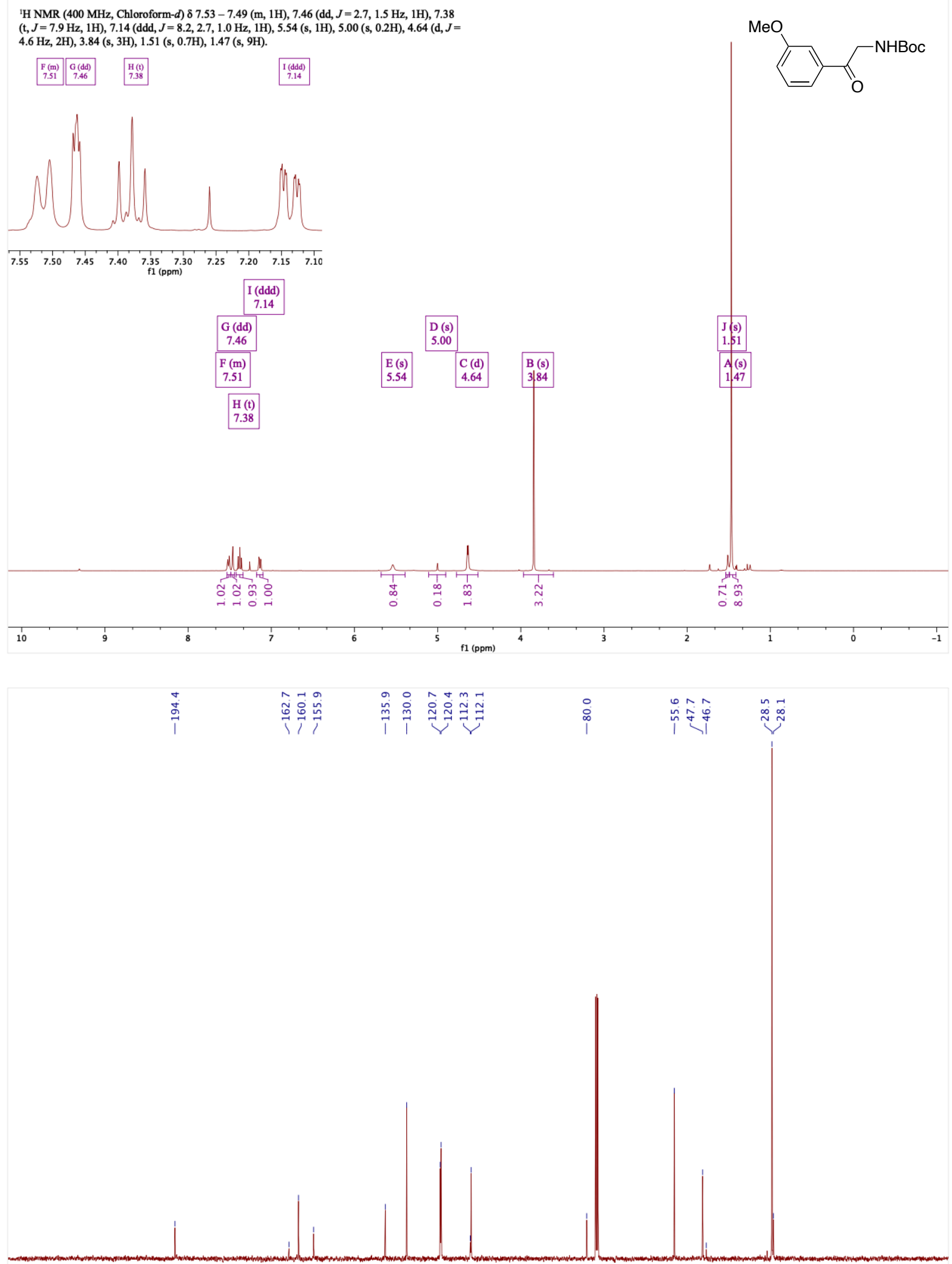

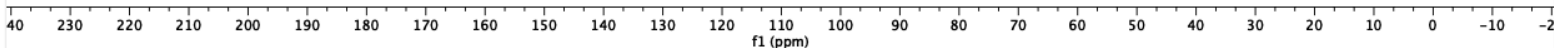


tert-Butyl (2-(3,4-dimethoxyphenyl)-2-oxoethyl)carbamate, $2 \mathrm{i}$ 'H NMR $(400 \mathrm{MHz}$, Chloroform- $d) \delta 7.57(\mathrm{dd}, J=8.5,2.0 \mathrm{~Hz}, 1 \mathrm{H}), 7.49(\mathrm{~d}, J=2.0 \mathrm{~Hz}, 1 \mathrm{H})$,
$6.90(\mathrm{~d}, J=8.4 \mathrm{~Hz}, 1 \mathrm{H}), 5.56(\mathrm{t}, J=4.6 \mathrm{~Hz}, 1 \mathrm{H}), 4.99(\mathrm{~s}, 0.1 \mathrm{H}), 4.62(\mathrm{~d}, J=4.5 \mathrm{~Hz}, 2 \mathrm{H}), 3.94$ $(\mathrm{s}, 3 \mathrm{H}), 3.92(\mathrm{~s}, 3 \mathrm{H}), 1.51(\mathrm{~s}, 0.4 \mathrm{H}), 1.47(\mathrm{~s}, 9 \mathrm{H})$.
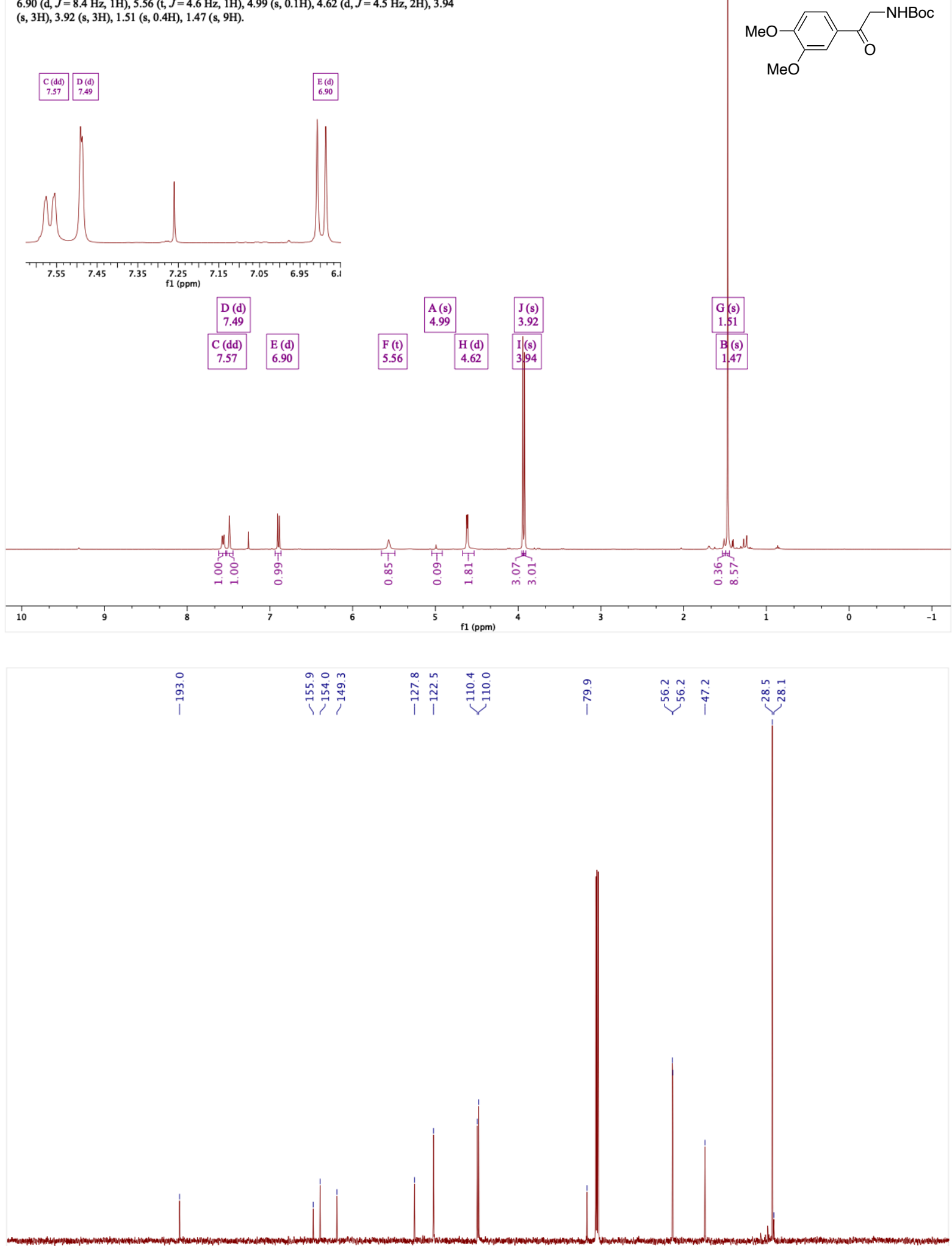

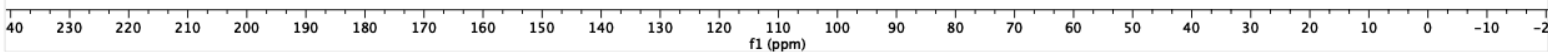


tert-Butyl (2-(2-methoxyphenyl)-2-oxoethyl)carbamate, $2 \mathrm{j}$ (non-hydrogen-bonded)

${ }^{1} \mathrm{H}$ NMR $(400 \mathrm{MHz}$, Chloroform- $d$ ) 87.94 (dd, $J=7.8,1.8 \mathrm{~Hz}, 1 \mathrm{H}), 7.51$ (ddd, $J=8.8,7.3,1.9$ $\mathrm{Hz}, 1 \mathrm{H}), 7.05-7.00(\mathrm{~m}, 1 \mathrm{H}), 6.98(\mathrm{~d}, J=8.4 \mathrm{~Hz}, 1 \mathrm{H}), 5.63(\mathrm{~s}, 1 \mathrm{H}), 4.61(\mathrm{~d}, J=4.8 \mathrm{~Hz}, 2 \mathrm{H})$, $3.93(\mathrm{~s}, 3 \mathrm{H}), 1.46(\mathrm{~s}, 9 \mathrm{H})$
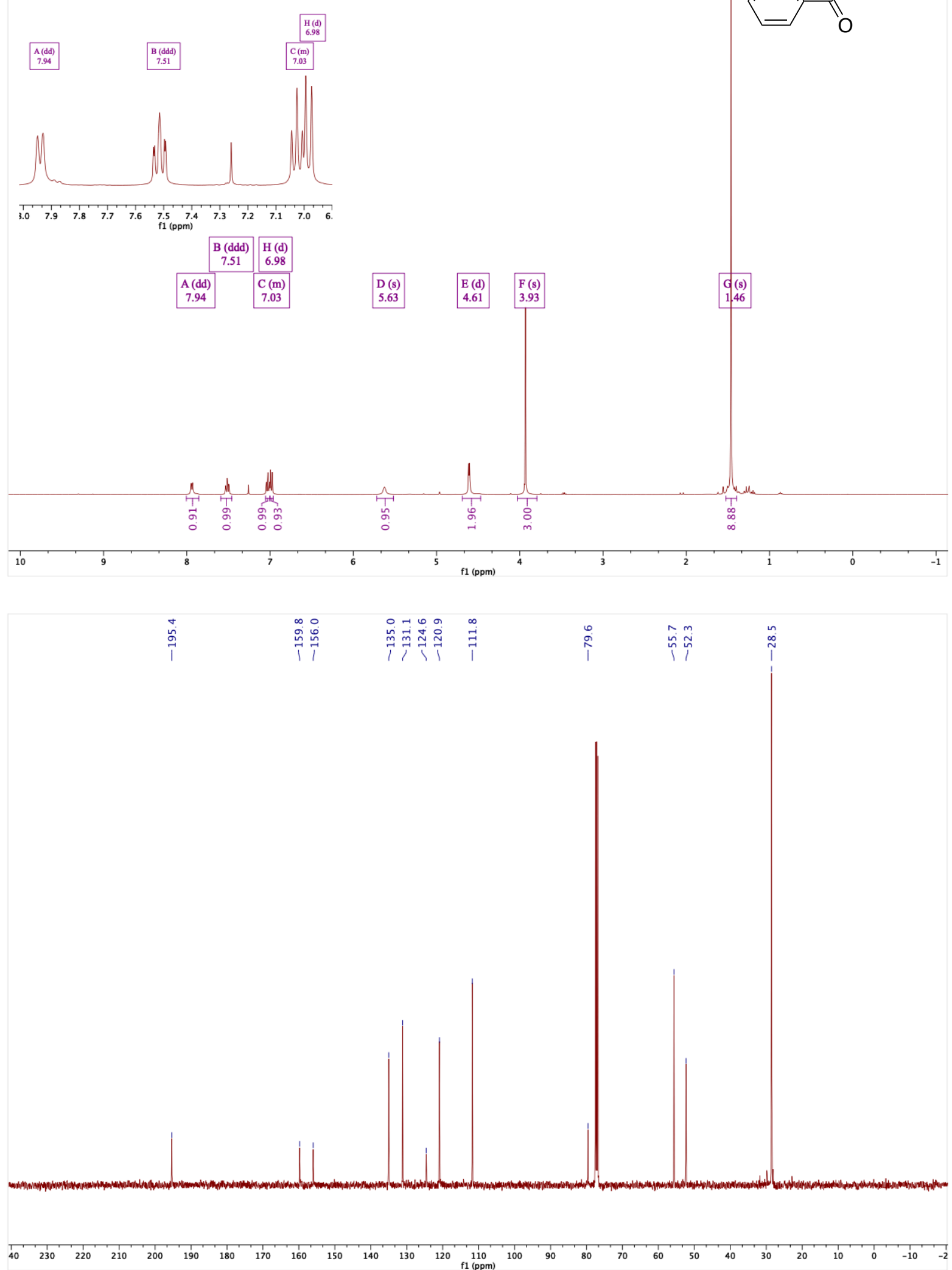
tert-Butyl (2-(2-methoxyphenyl)-2-oxoethyl)carbamate, 2j' (hydrogen-bonded)
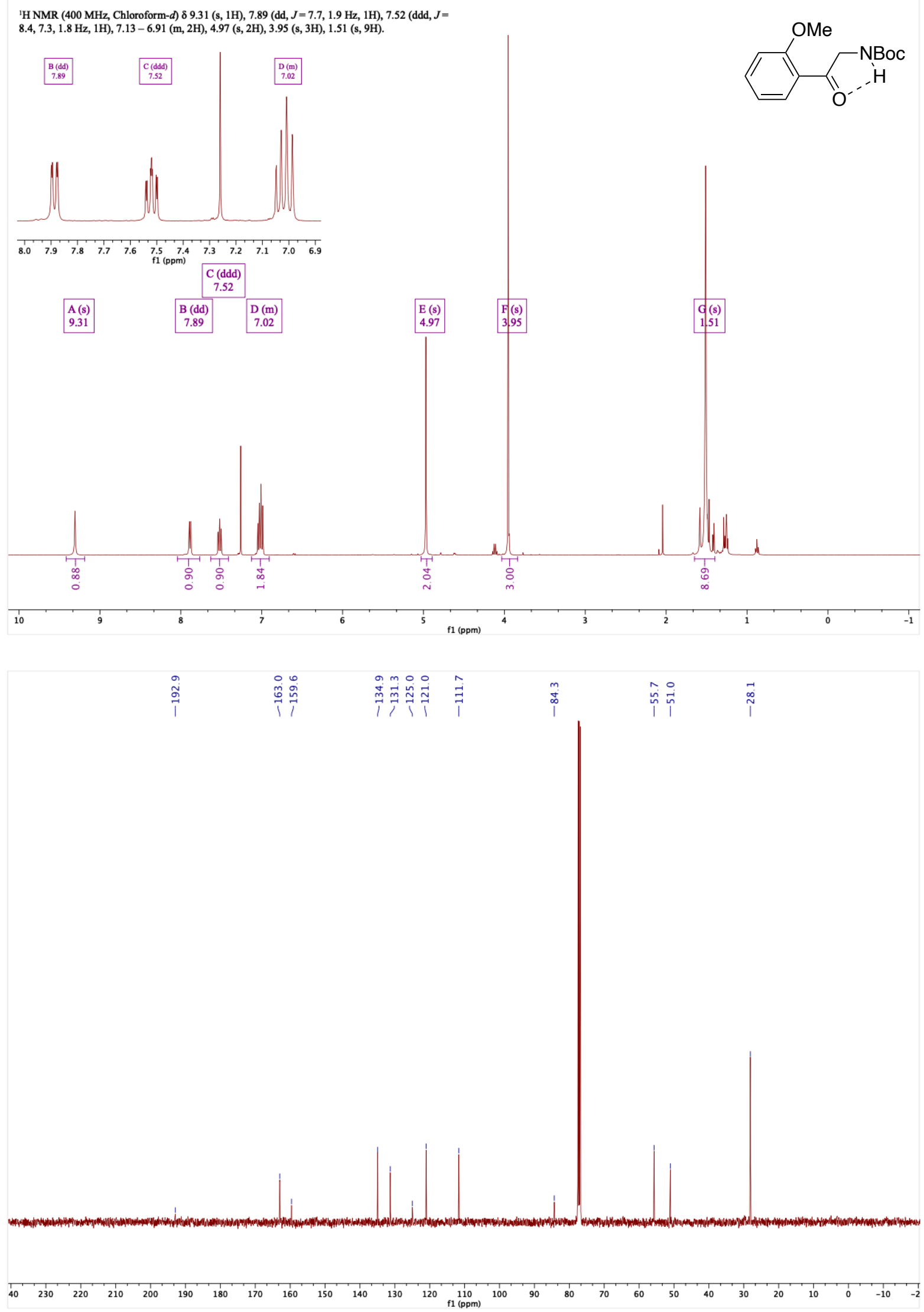
tert-Butyl (2-(4-chlorophenyl)-2-oxoethyl)carbamate, 21

${ }^{1} \mathrm{H}$ NMR $(400 \mathrm{MHz}$, Chloroform- $d$ ) $89.30(\mathrm{~s}, 1 \mathrm{H}), 7.96-7.84(\mathrm{~m}, 2 \mathrm{H}), 7.53-7.42(\mathrm{~m}, 2 \mathrm{H})$ $4.99(\mathrm{~s}, 2 \mathrm{H}), 4.62(\mathrm{~d}, J=4.6 \mathrm{~Hz}, 0.1 \mathrm{H}), 1.52(\mathrm{~s}, 9 \mathrm{H}), 1.47(\mathrm{~s}, 0.5 \mathrm{H})$.
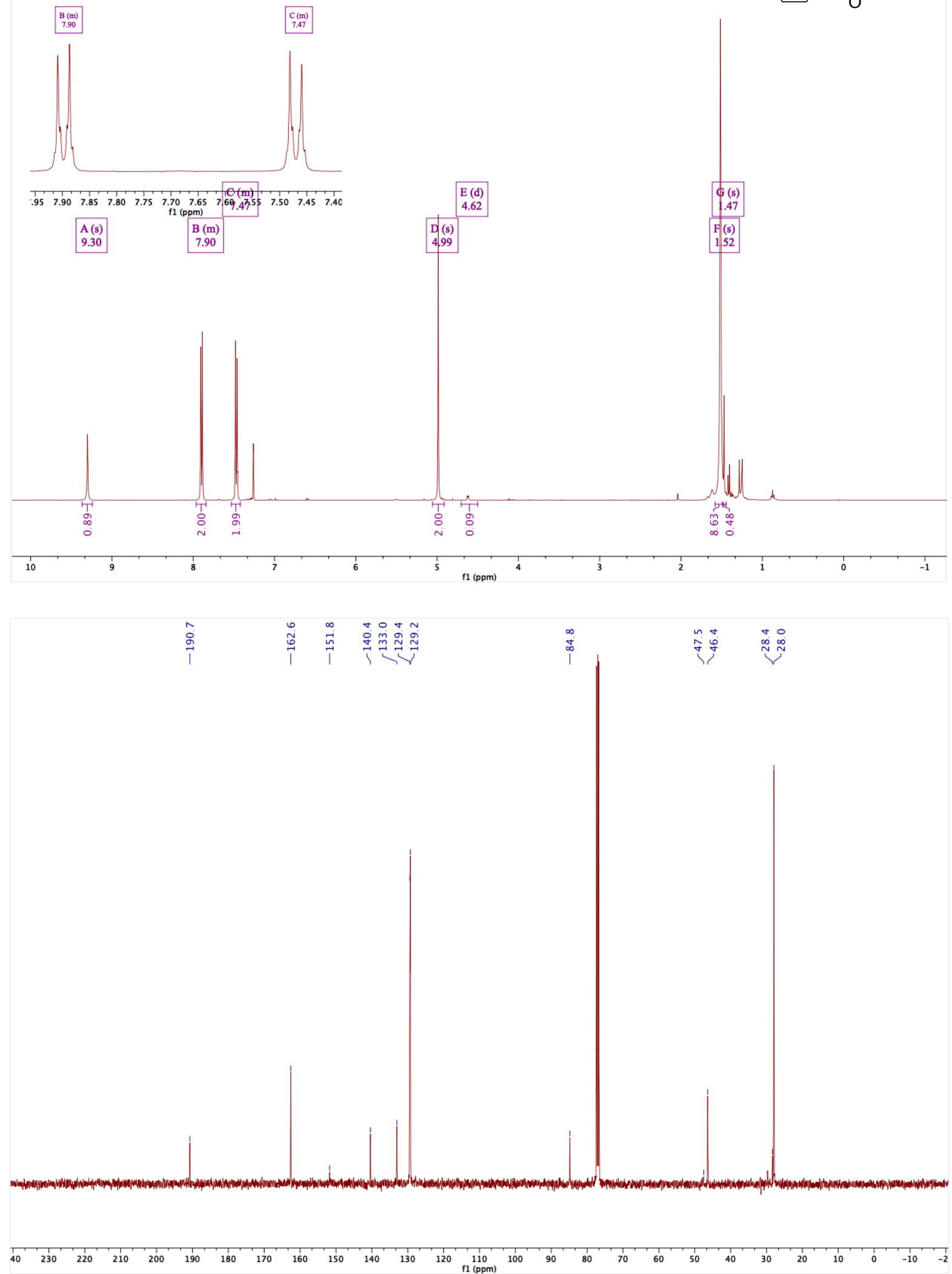
tert-Butyl (2-oxo-2-(4-(trifluoromethyl)phenyl)ethyl)carbamate, $2 \mathrm{~m}$
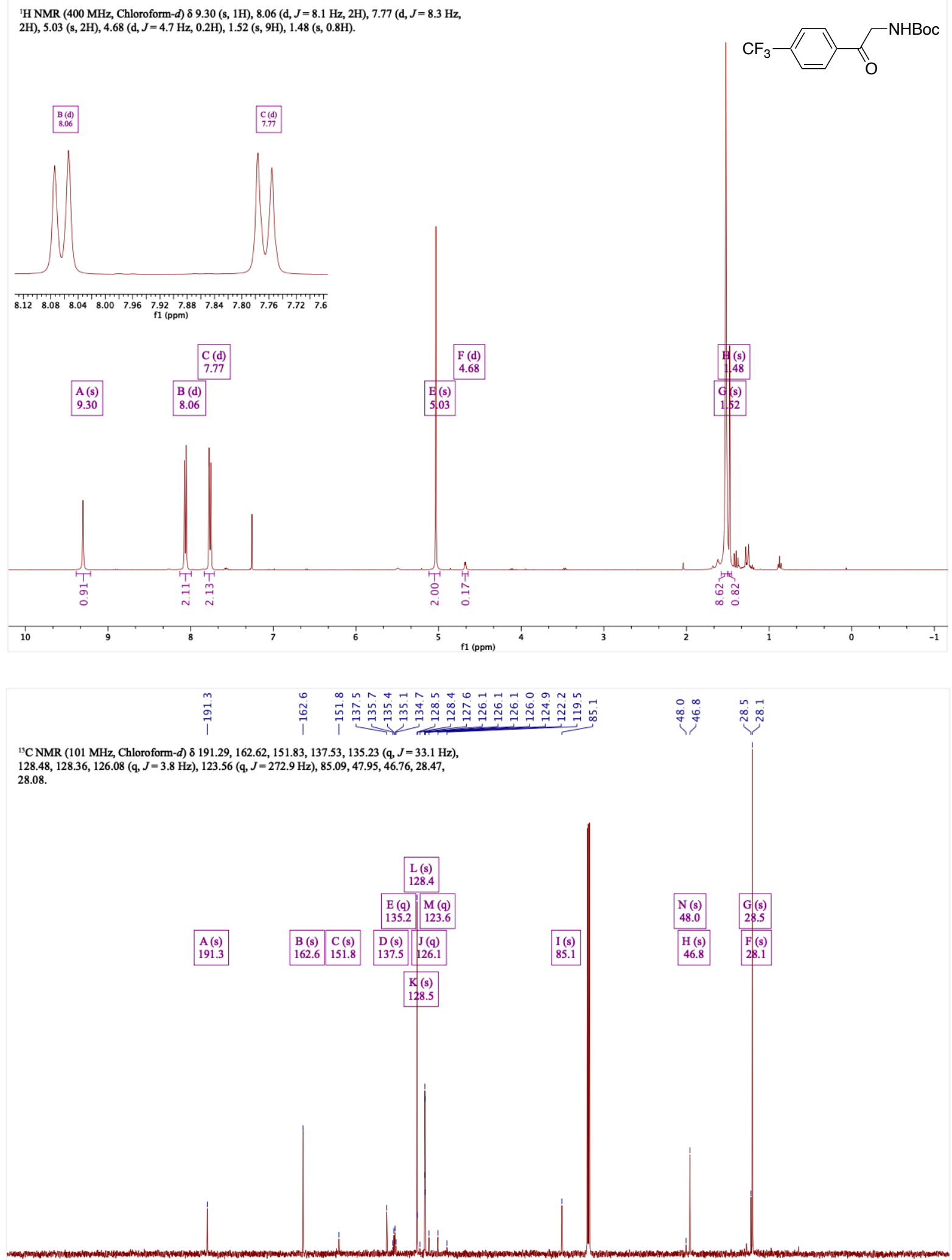

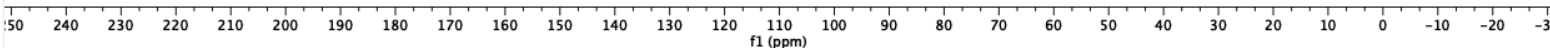


${ }^{19} \mathrm{~F}$ NMR (377 MHz, Chloroform- $d$ ) $\delta$-63.24, -63.25.

구유ำ

అִ

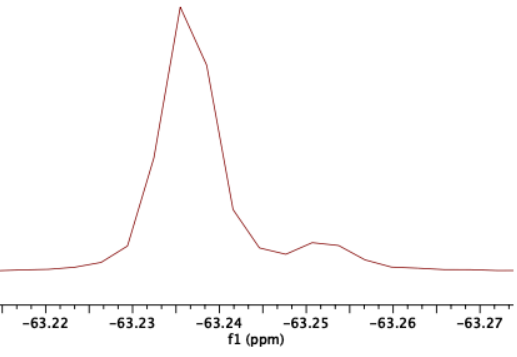

\begin{tabular}{|c|}
\hline $\mathbf{B}(\mathrm{s})$ \\
\hline-63.25 \\
\hline $\mathbf{A}(\mathbf{s})$ \\
-63.24 \\
\hline
\end{tabular}

80

$\begin{array}{llll}1 & 1 & 1 & 1 \\ 60 & 40 & 20 & 0\end{array}$

$-20$

$-60$

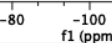


tert-Butyl (2-(benzo[b]thiophen-3-yl)-2-oxoethyl)carbamate, $2 \mathrm{n}$

${ }^{1} \mathrm{H}$ NMR $(400 \mathrm{MHz}$, Chloroform- $d$ ) $\delta 9.35$ (s, $0.2 \mathrm{H}$ ), 8.71 (ddd, $J=8.1,1.3,0.7 \mathrm{~Hz}, 0.7 \mathrm{H}$ ), 8.68 (ddd, $J=8.1,1.4,0.8 \mathrm{~Hz}, 0.2 \mathrm{H}), 8.36(\mathrm{~s}, 0.2 \mathrm{H}), 8.35(\mathrm{~s}, 0.8 \mathrm{H}), 7.93-7.84(\mathrm{~m}, 1 \mathrm{H}), 7.50(\mathrm{dtd}, J$
$=8.3,7.1,1.3 \mathrm{~Hz}, 1 \mathrm{H}), 7.43(\mathrm{ddt}, J=9.0,7.2,1.6 \mathrm{~Hz}, 1 \mathrm{H}), 5.57(\mathrm{~s}, 1 \mathrm{H}), 5.04(\mathrm{~s}, 0.5 \mathrm{H}), 4.65(\mathrm{~d}$, $J=4.8 \mathrm{~Hz}, 1.5 \mathrm{H}), 1.52(\mathrm{~d}, J=1.6 \mathrm{~Hz}, 2.7 \mathrm{H}), 1.49(\mathrm{~s}, 7.3 \mathrm{H})$.

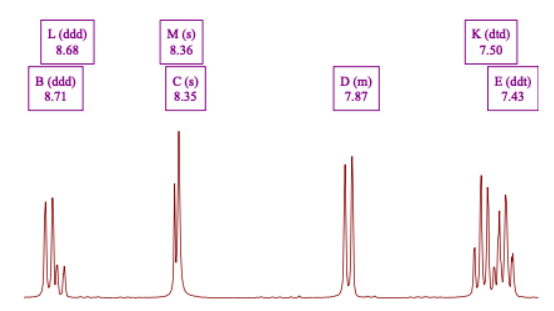

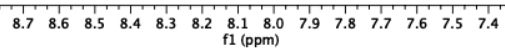

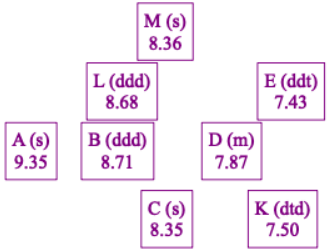
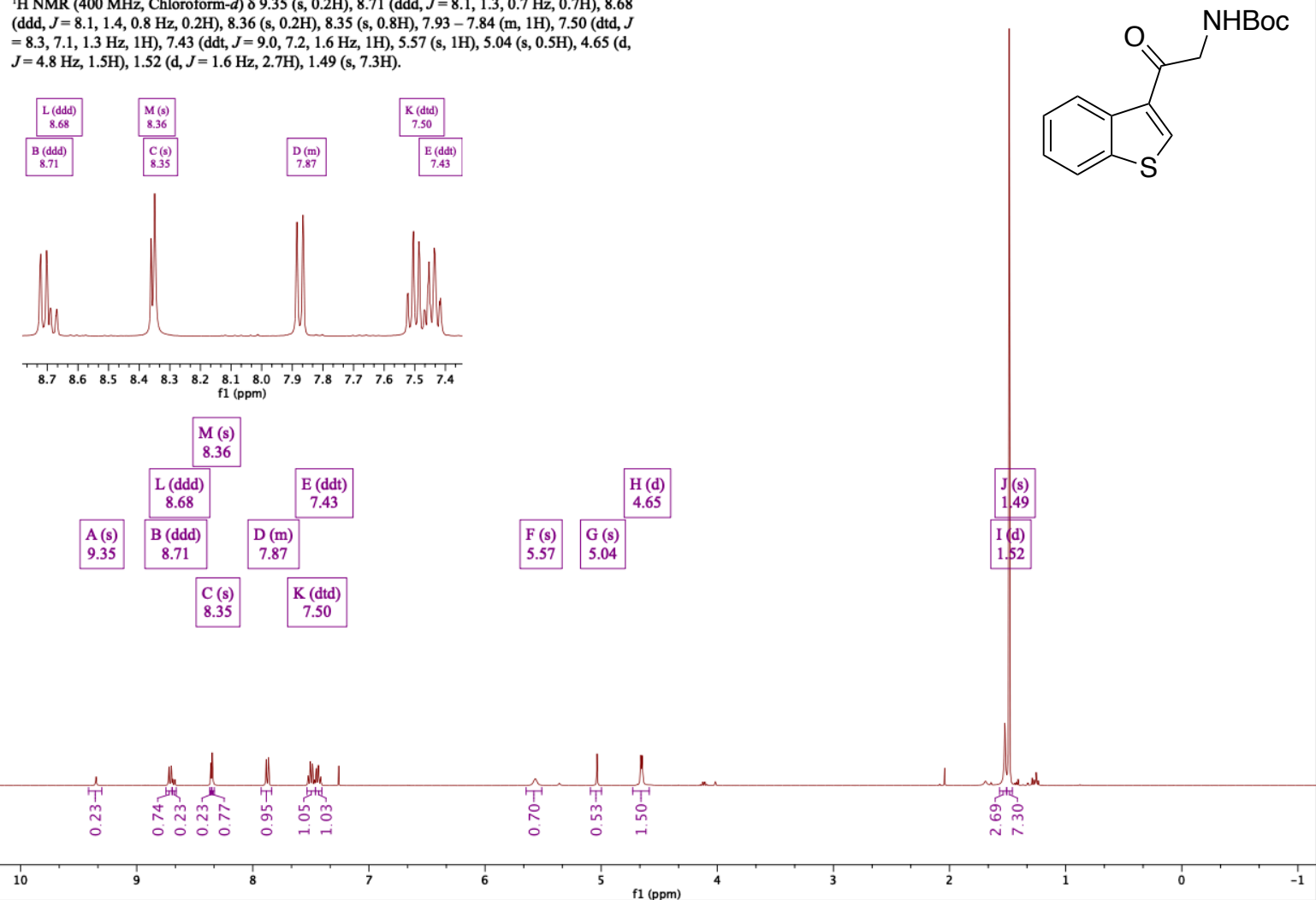

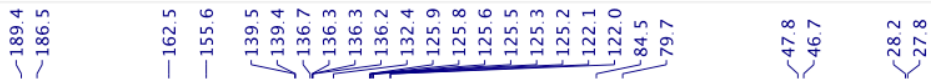




\section{tert-Butyl (2-oxo-2-(thiophen-2-yl)ethyl)carbamate, 20}

${ }^{1} \mathrm{H}$ NMR $(400 \mathrm{MHz}$, Chloroform- $d) \delta 9.29(\mathrm{~s}, 0 \mathrm{H}), 7.78(\mathrm{dd}, J=3.9,1.1 \mathrm{~Hz}, 0 \mathrm{H}), 7.76(\mathrm{dd}, J=$
$3.8,1.1 \mathrm{~Hz}, 1 \mathrm{H}), 7.69(\mathrm{dd}, J=5.0,1.1 \mathrm{~Hz}, 0 \mathrm{H}), 7.68(\mathrm{dd}, J=5.0,1.1 \mathrm{~Hz}, 1 \mathrm{H}), 7.19-7.12(\mathrm{~m}$,

$1 \mathrm{H}), 5.44(\mathrm{~s}, 1 \mathrm{H}), 4.95(\mathrm{~s}, 1 \mathrm{H}), 4.57(\mathrm{~d}, J=4.8 \mathrm{~Hz}, 1 \mathrm{H}), 1.50(\mathrm{~s}, 3 \mathrm{H}), 1.46(\mathrm{~s}, 7 \mathrm{H})$.
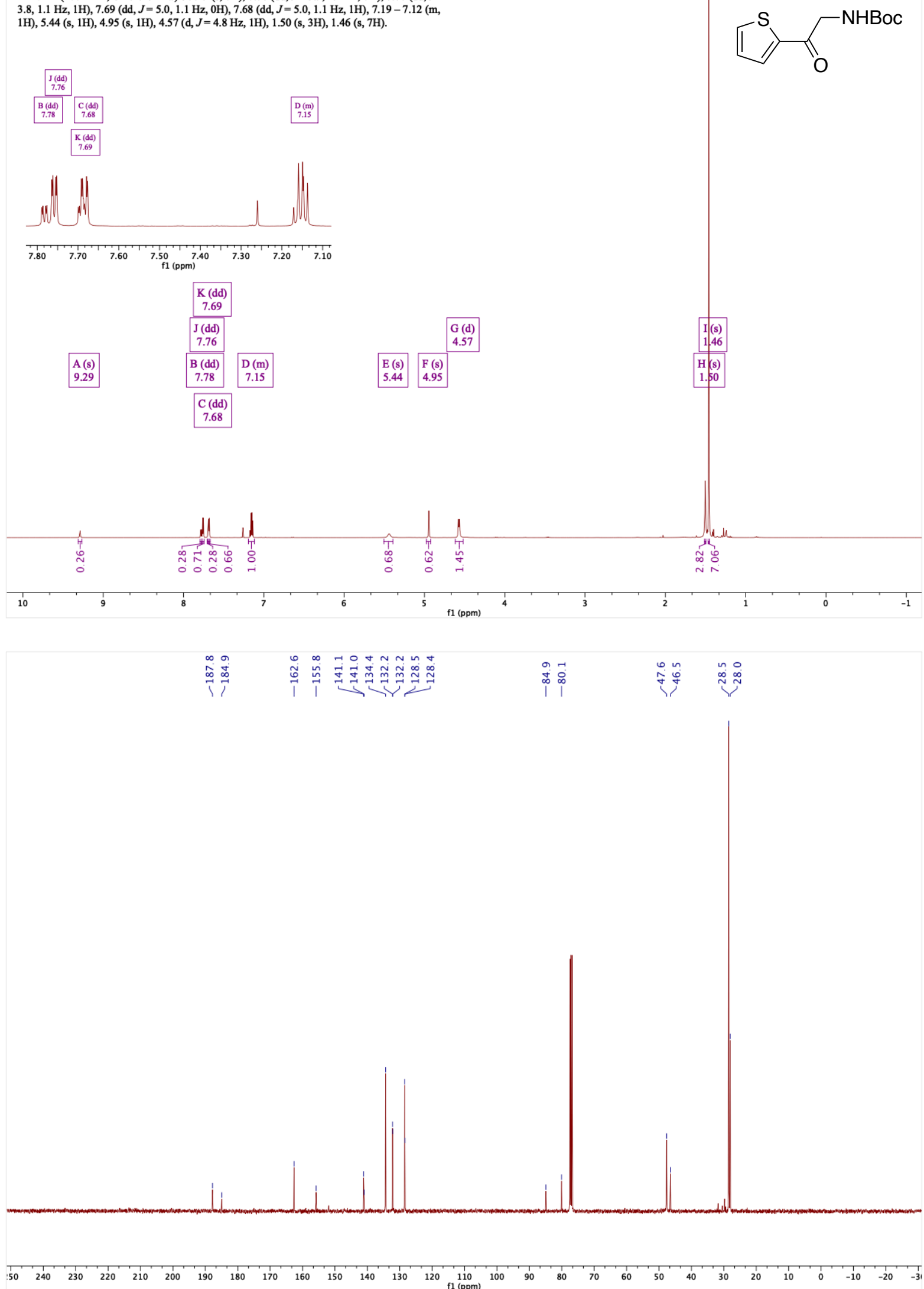


\section{tert-Butyl (2-oxo-2-(1-tosyl-1H-indol-2-yl)ethyl)carbamate, $2 \mathrm{p}$}

${ }^{1} \mathrm{H}$ NMR $(400 \mathrm{MHz}$, Chloroform- $d) \delta 9.30(\mathrm{~s}, 0 \mathrm{H}), 8.18-8.09(\mathrm{~m}, 1 \mathrm{H}), 7.90-7.86(\mathrm{~m}, 0 \mathrm{H})$,
$7.86-7.81(\mathrm{~m}, 2 \mathrm{H}), 7.63-7.53(\mathrm{~m}, 1 \mathrm{H}), 7.50-7.43(\mathrm{~m}, 1 \mathrm{H}), 7.32-7.26(\mathrm{~m}, 1 \mathrm{H}), 7.26-7.21$ $7.86-7.81(\mathrm{~m}, 2 \mathrm{H}), 7.63-7.53(\mathrm{~m}, 1 \mathrm{H}), 7.50-7.43(\mathrm{~m}, 1 \mathrm{H}), 7.32-7.26(\mathrm{~m}, 1 \mathrm{H}), 7.26-7.2$ $2 \mathrm{H}), 1.43(\mathrm{~s}, 8 \mathrm{H})$
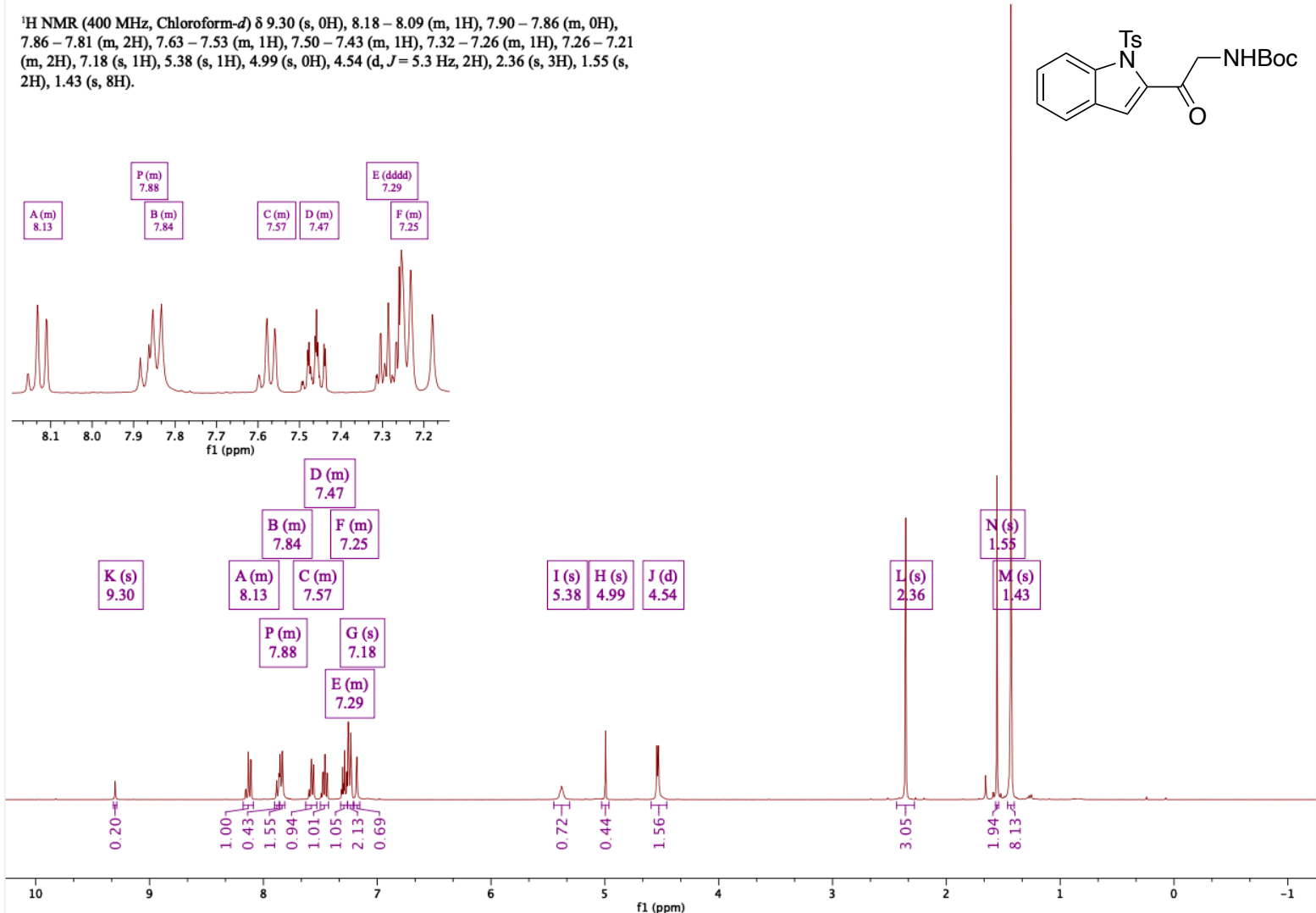

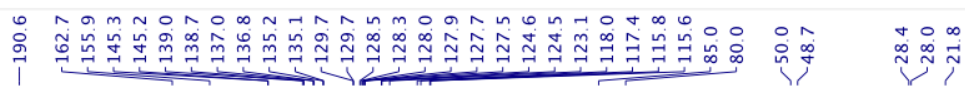

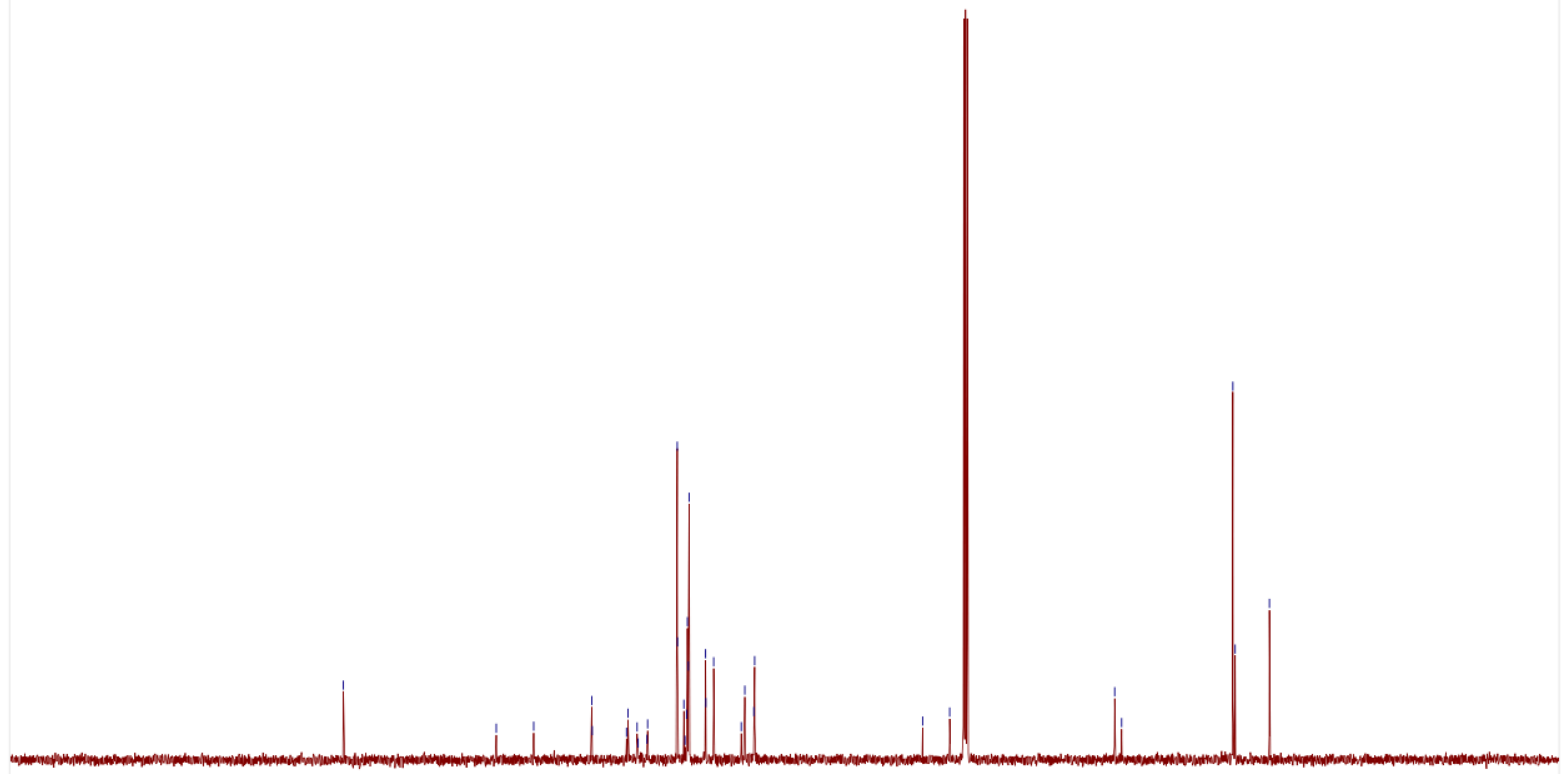

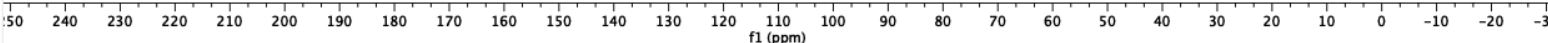


tert-Butyl 3-(4-fluorophenyl)-3-hydroxypyrrolidine-1-carboxylate, 3

${ }^{1} \mathrm{H}$ NMR (400 MHz, Chloroform- $d$ ) $87.54-7.42(\mathrm{~m}, 2 \mathrm{H}), 7.12-7.02(\mathrm{~m}, 2 \mathrm{H}), 3.83-3.48$ (m, $4 \mathrm{H}), 2.40-2.10(\mathrm{~m}, 3 \mathrm{H}), 1.49(\mathrm{~d}, J=6.2 \mathrm{~Hz}, 9 \mathrm{H})$.
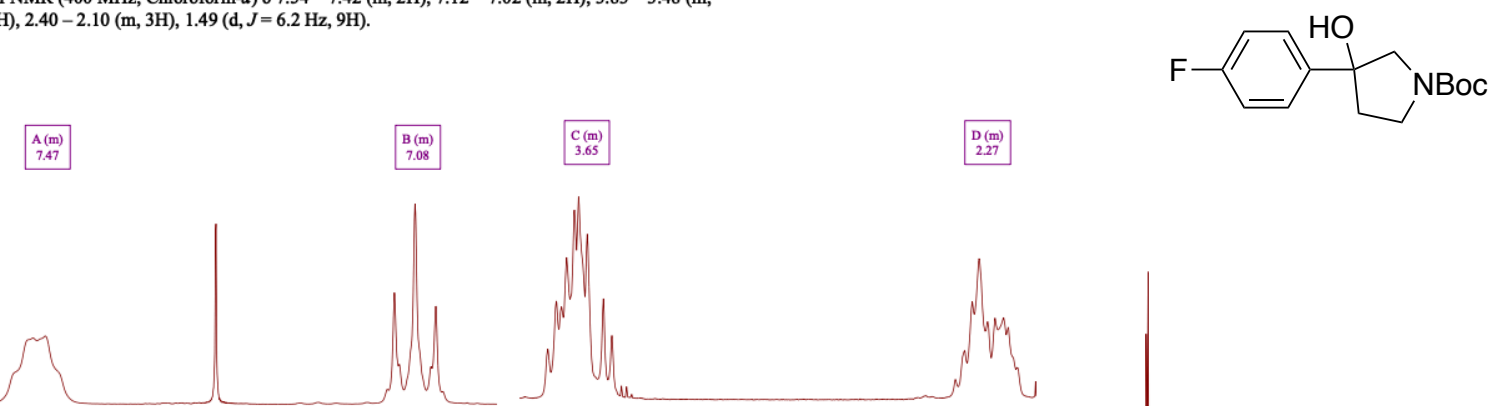

\begin{tabular}{l|l}
\hline $\mathbf{c}(\mathrm{m})$ \\
3.65 \\
\hline
\end{tabular}

\begin{tabular}{|l|}
\hline $\mathbf{D}(\mathrm{m})$ \\
2.27 \\
\hline
\end{tabular}

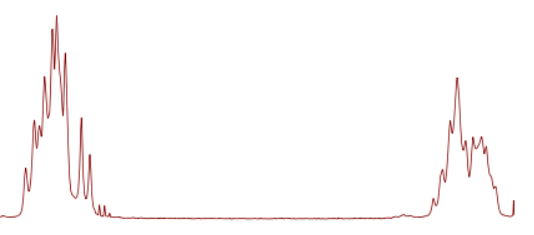

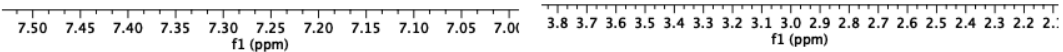

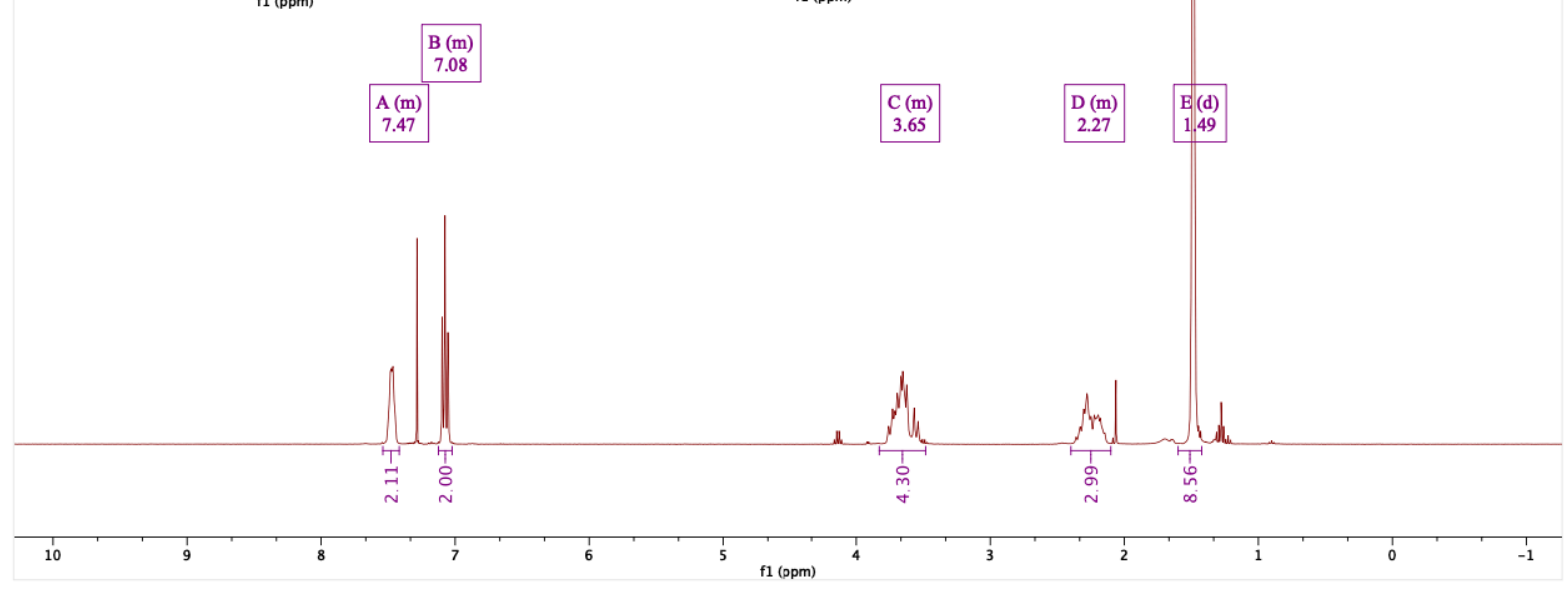

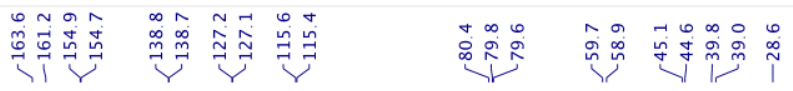

${ }^{13} \mathrm{C}$ NMR $(101 \mathrm{MHz}$, Chloroform- $d$ ) $\delta 162.38$ (d, $J=246.6 \mathrm{~Hz}), 154.87,154.74,138.74$ (d, $J=$ $8.3 \mathrm{~Hz}), 127.16(\mathrm{~d}, J=8.2 \mathrm{~Hz}), 115.50(\mathrm{~d}, J=21.3 \mathrm{~Hz}), 80.40,79.75,79.57,59.67,58.94$ $45.13,44.65,39.81,39.03,28.65$.

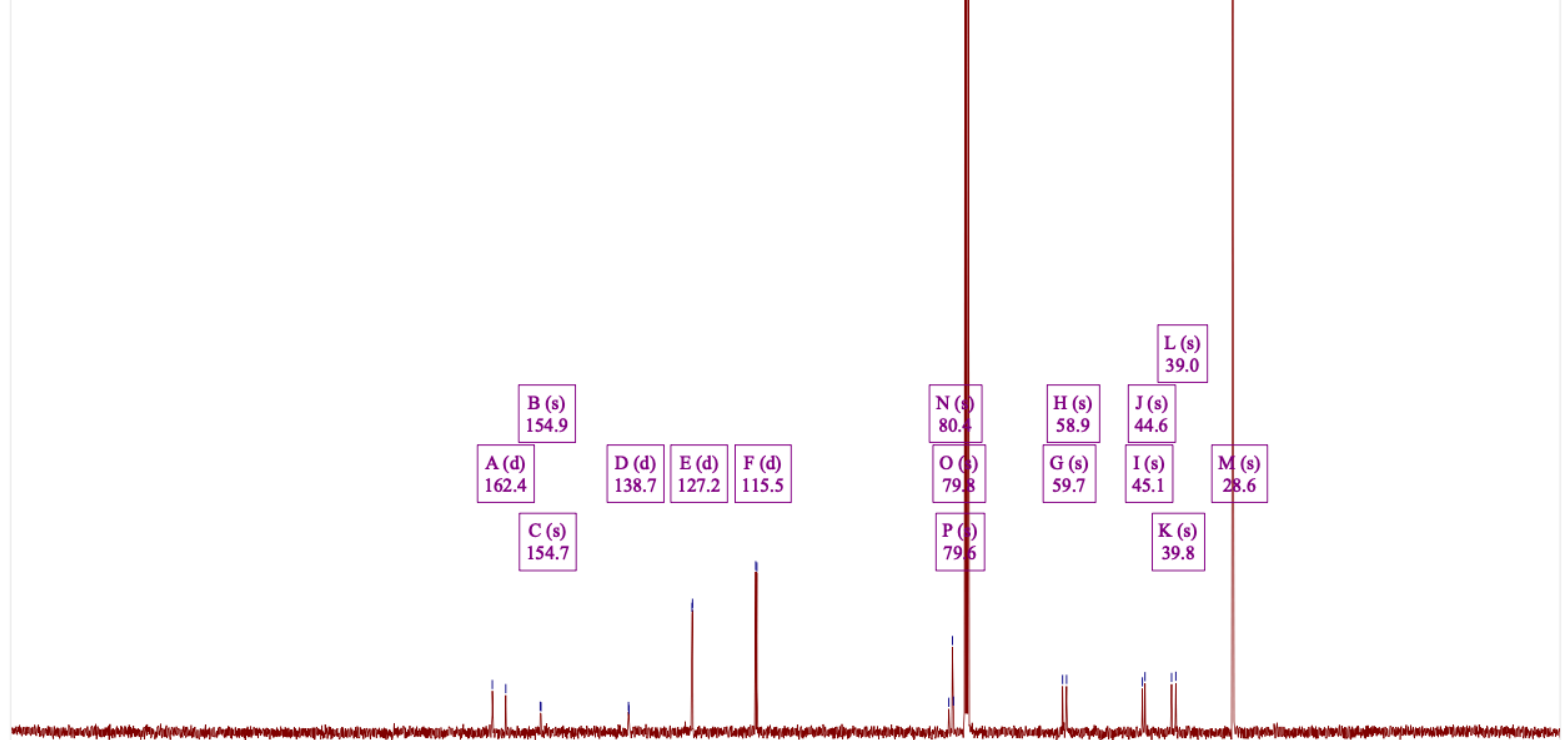


${ }^{19} \mathrm{~F}$ NMR (377 MHz, Chloroform- $d$ ) $\delta$-114.60 - -114.91 (m)

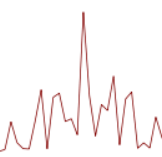

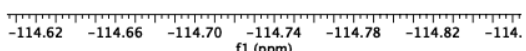

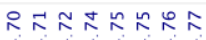

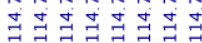

$\mathrm{A}(\mathrm{m})$
-114.74

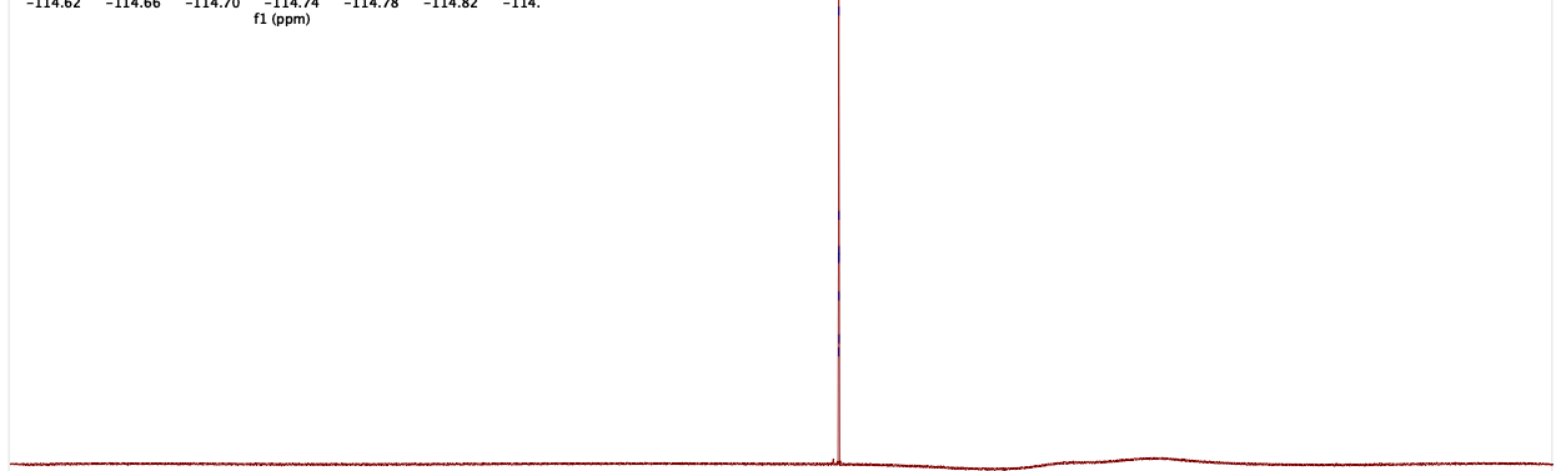

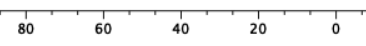

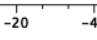

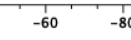

$\mathrm{f}^{-100}(\mathrm{ppm})$

$-120 \quad-140$

$-160$

$-220$

$-260+2$ $-280$ 
tert-Butyl (3-(4-fluorophenyl)-3-oxopropyl)carbamate, 4
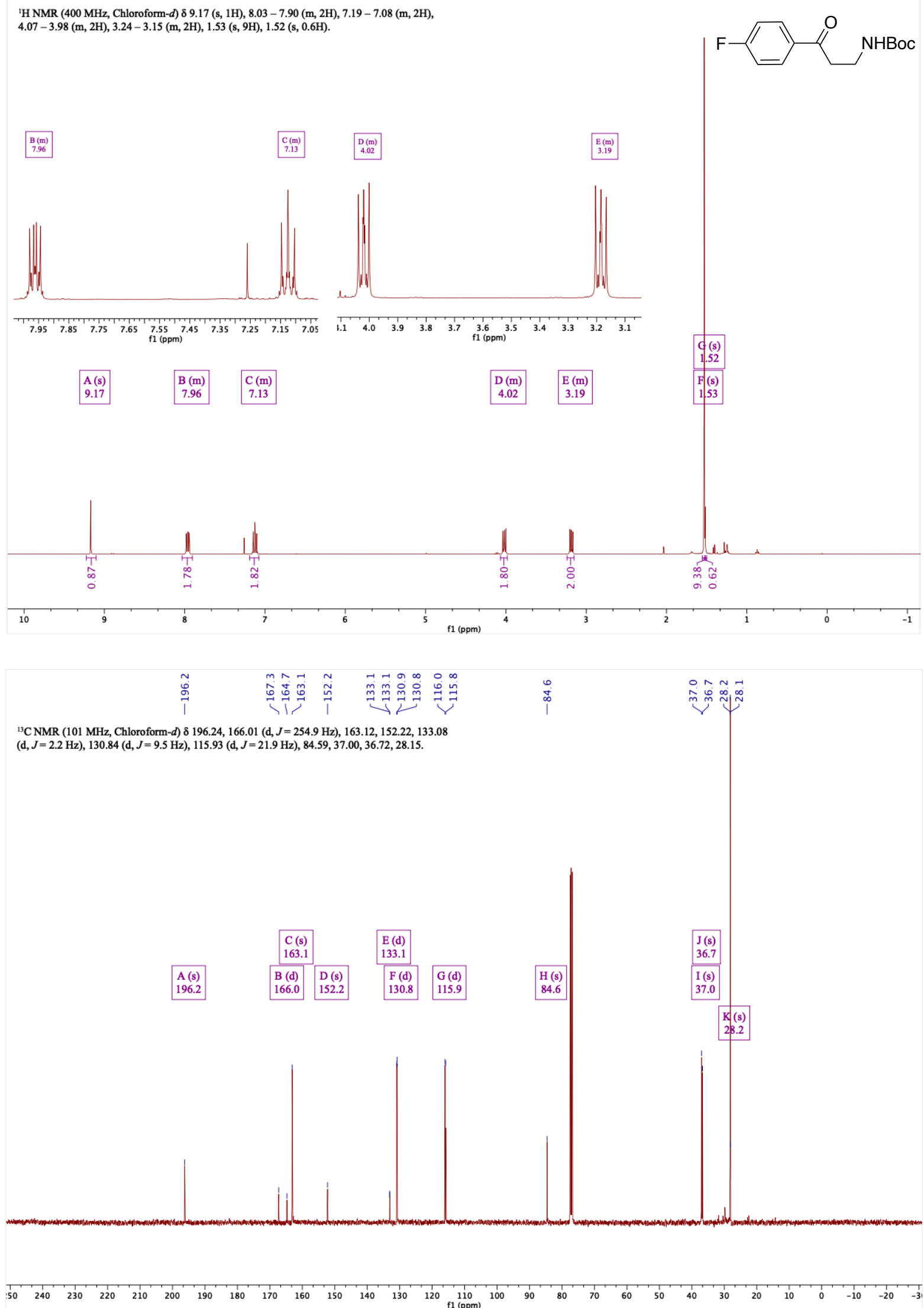
${ }^{19} \mathrm{~F}$ NMR (377 MHz, Chloroform- $d$ ) $\delta$-104.64 - -104.92 (m).

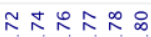

总㤩总总总

A (m)

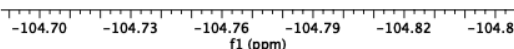


tert-Butyl 3-(4-fluorophenyl)-3-methoxyazetidine-1-carboxylate, 5

${ }^{1} \mathrm{H}$ NMR (400 MHz, Chloroform- $d$ ) $87.39-7.33$ (m, 2H), $7.12-7.05$ (m, 2H), 4.16 (d, $J=8.9$ $\mathrm{Hz}, 2 \mathrm{H}), 4.12(\mathrm{~d}, J=8.9 \mathrm{~Hz}, 2 \mathrm{H}), 3.07(\mathrm{~s}, 3 \mathrm{H}), 1.45(\mathrm{~s}, 9 \mathrm{H})$
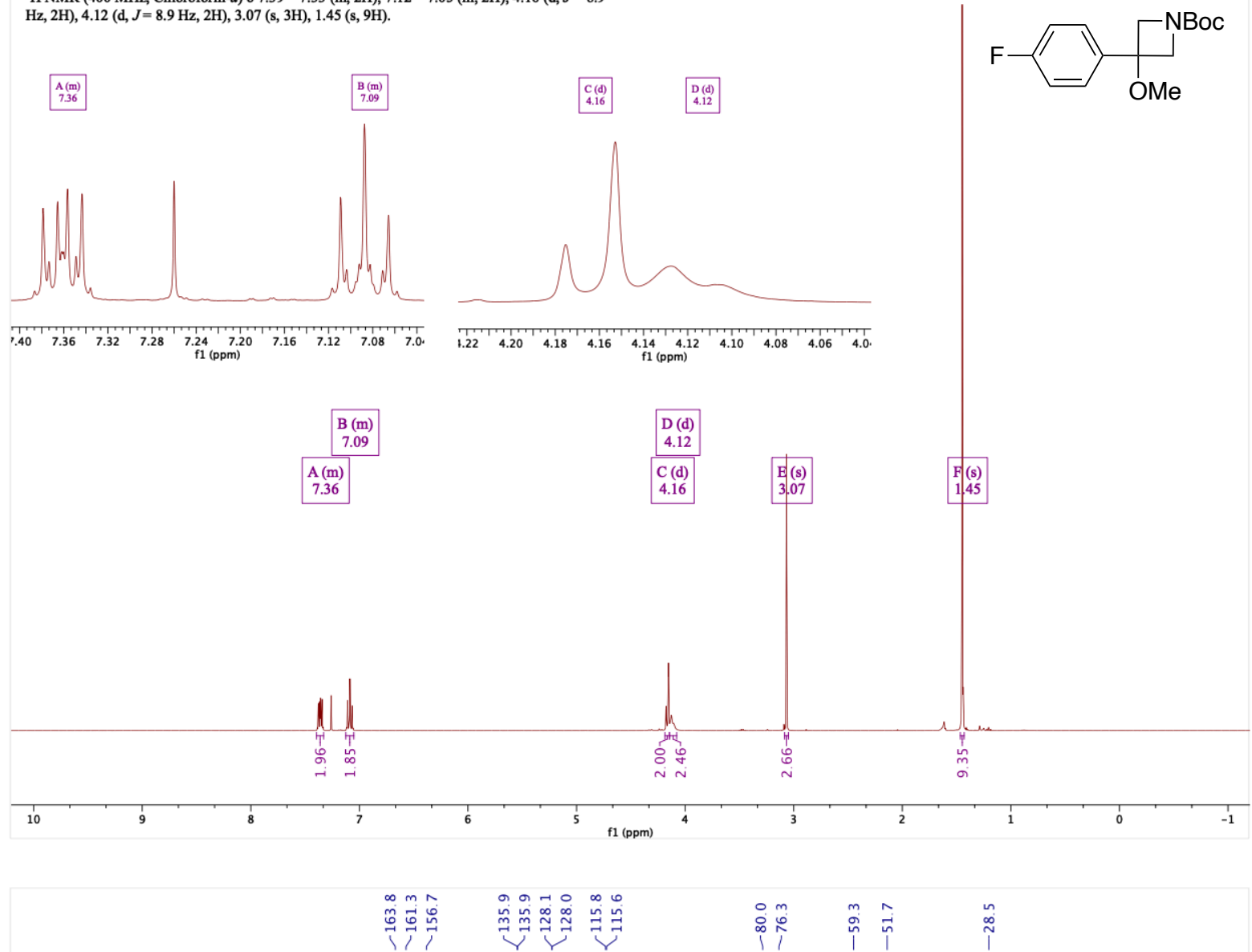

${ }^{13} \mathrm{C}$ NMR $(101 \mathrm{MHz}$, Chloroform- $d$ ) $\delta 162.54$ (d, $J=247.3 \mathrm{~Hz}), 156.66,135.93(\mathrm{~d}, J=3.3 \mathrm{~Hz})$ $128.06(\mathrm{~d}, J=8.2 \mathrm{~Hz}), 115.73(\mathrm{~d}, J=21.6 \mathrm{~Hz}), 80.01,76.32,59.31,51.68,28.50$

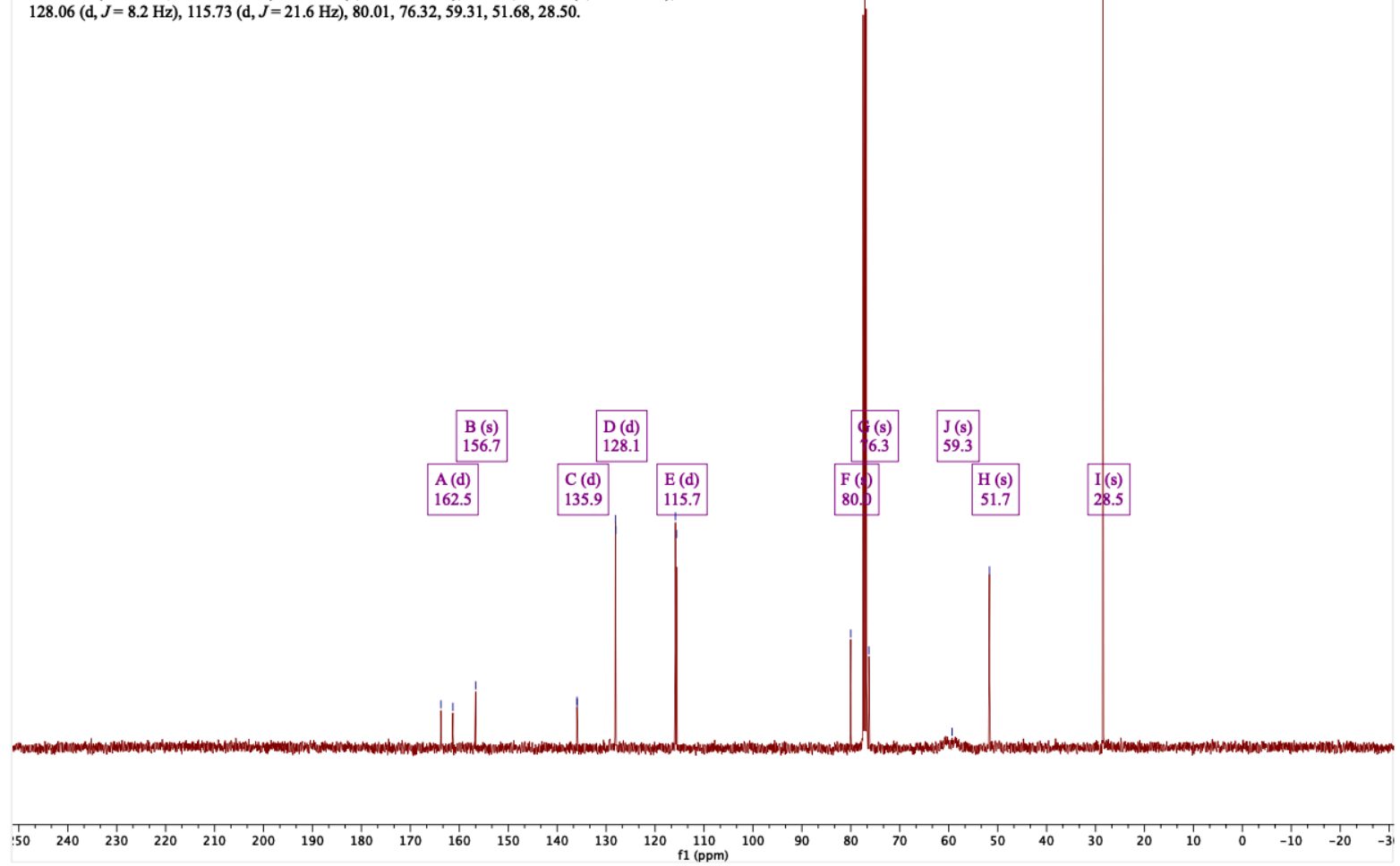


${ }^{19} \mathrm{~F}$ NMR (377 MHz, Chloroform- $\left.d\right) \delta-107.44--121.61(\mathrm{~m})$

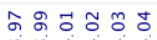

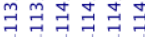

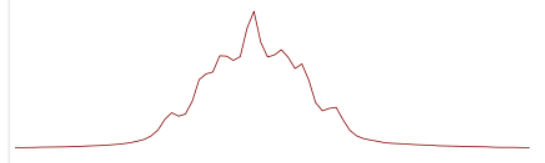

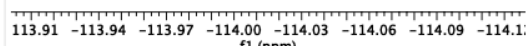

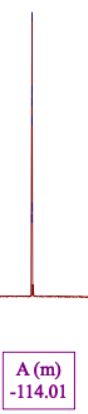

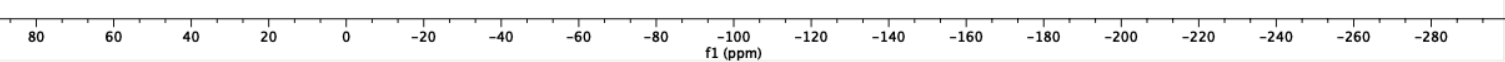


di-tert-Butyl ethane-1,2-diylbis((2-(benzo[b]thiophen-2-yl)-2-oxoethyl)carbamate), 6

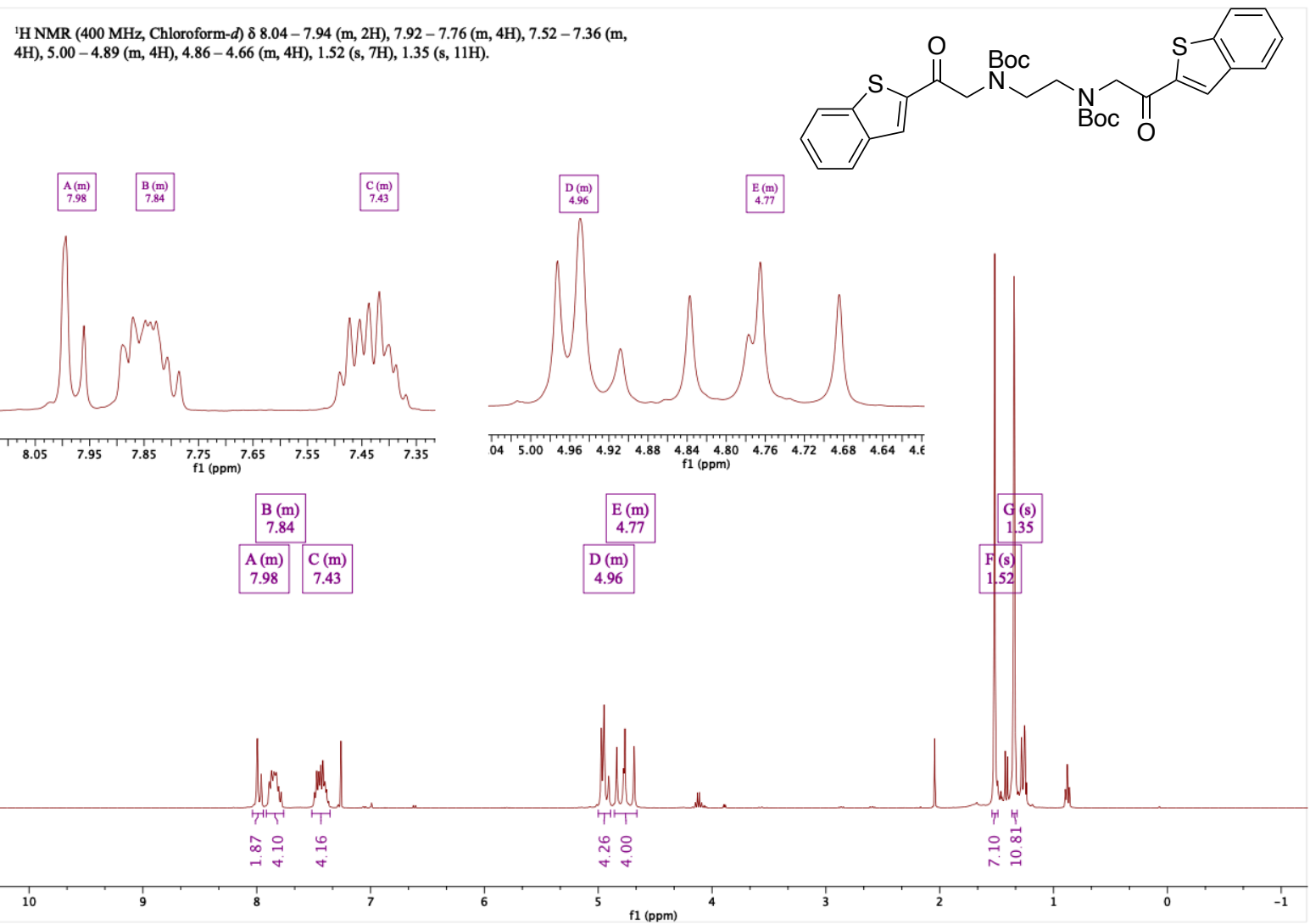

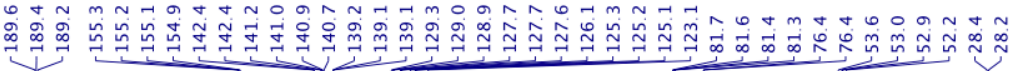

${ }^{13} \mathrm{C}$ NMR (101 MHz, Chloroform- $d$ ) $\delta 190.06-188.83$ (m), $155.72-154.45$ (m), 142.40 (d, $J=$ $5.7 \mathrm{~Hz}), 141.55-140.56(\mathrm{~m}), 139.35-138.95(\mathrm{~m}), 129.50-128.68(\mathrm{~m}), 127.92-127.29(\mathrm{~m})$, $126.10,125.60$ - 124.87 (m), 123.07, 82.06 - 80.99 (m), 76.58 - 76.01 (m), $54.59-51.79$ (m), $28.43,28.16$.
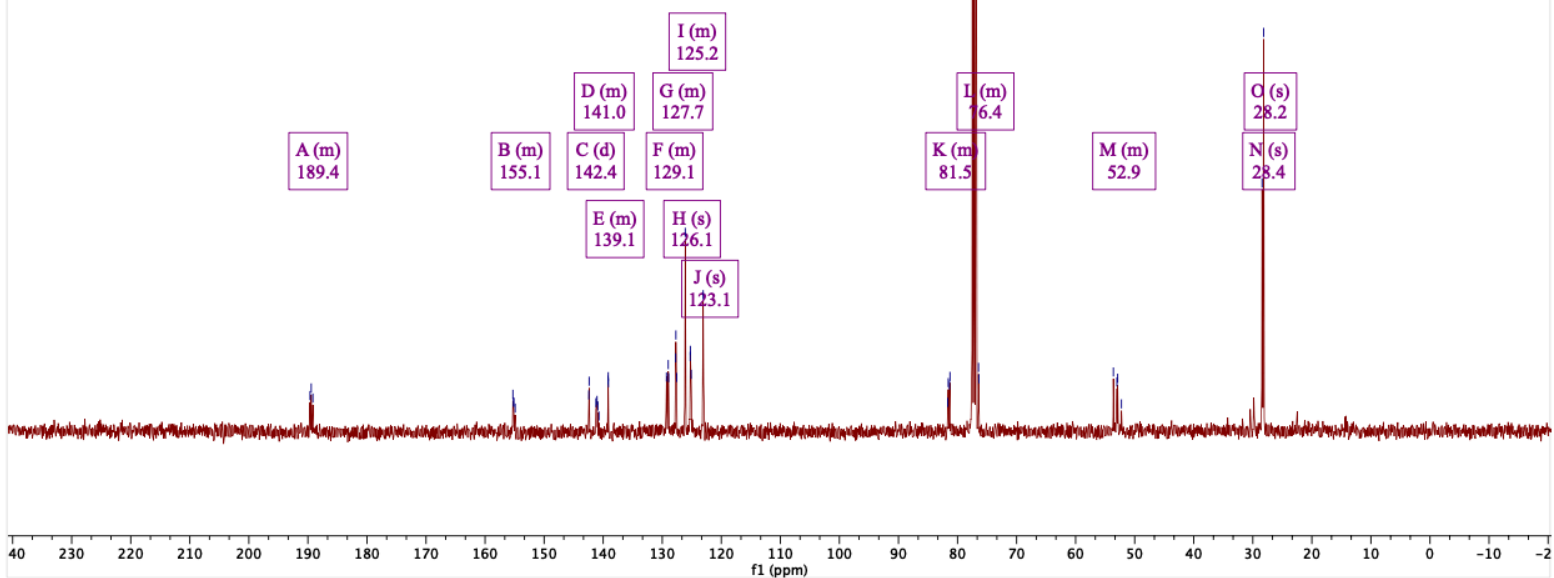


\section{Deuterated Solvent Experiment}

The conversion of $\mathbf{1 c}$ to $\mathbf{2 c}$ was performed through general procedure $(\mathrm{B})\left(\mathrm{d}_{2}\right.$-DCM instead of DCE, $\mathrm{D}_{2} \mathrm{O}$ instead of $\mathrm{H}_{2} \mathrm{O}$ ), and the ${ }^{1} \mathrm{H}$ and ${ }^{19} \mathrm{~F}$ NMR spectra of the aqueous layer (diluted with $\mathrm{D}_{2} \mathrm{O}$ ) and the organic layer (diluted with chloroform- $d$ ) were recorded after nine hours. No by-product structure could be identified by analysis of these spectra.

\section{Aqueous Layer:}

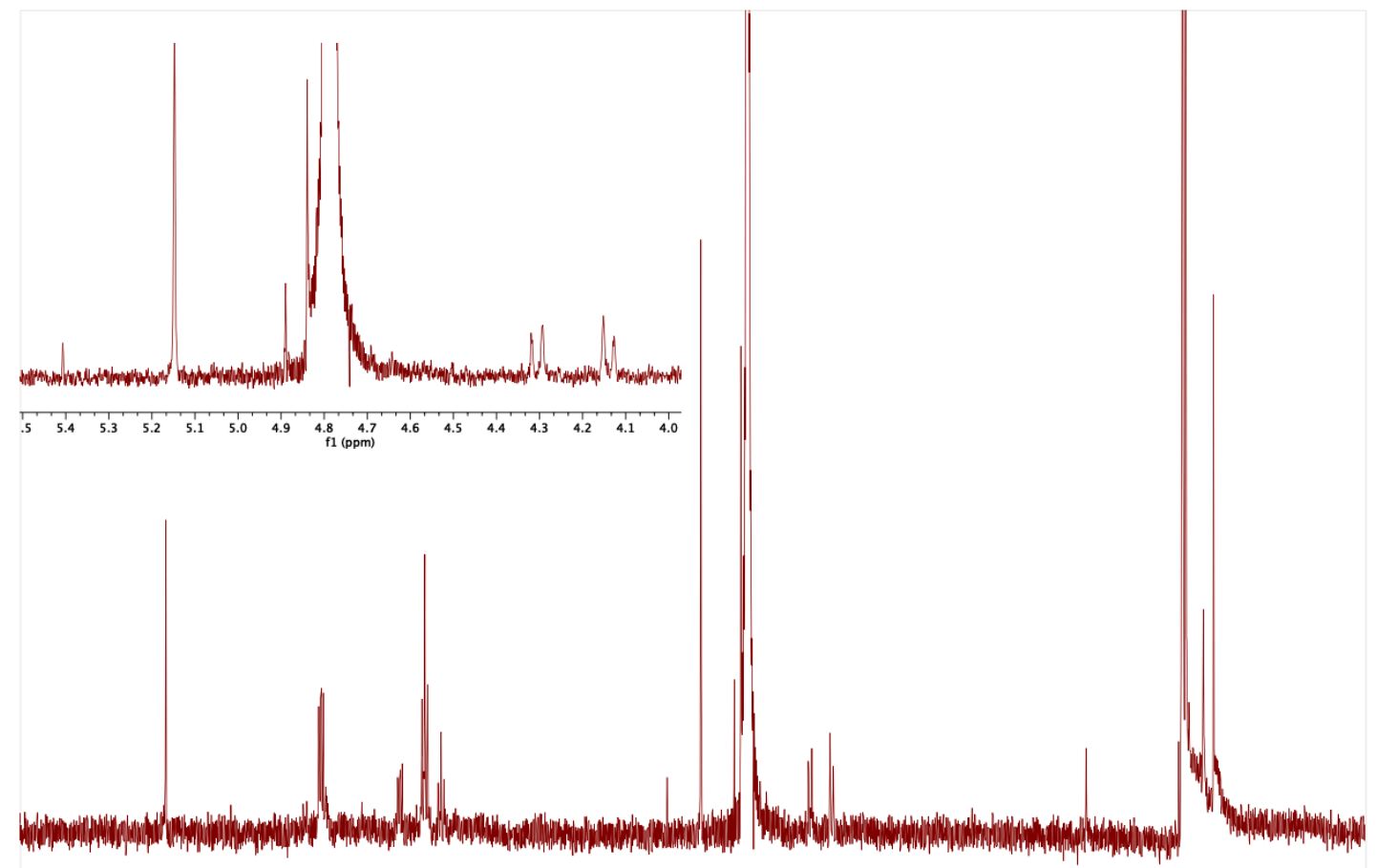

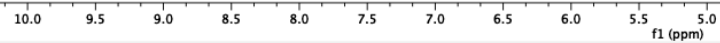

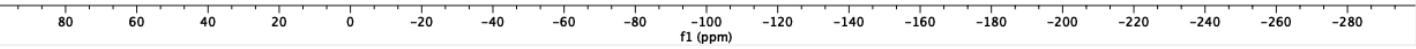




\section{Organic Layer:}
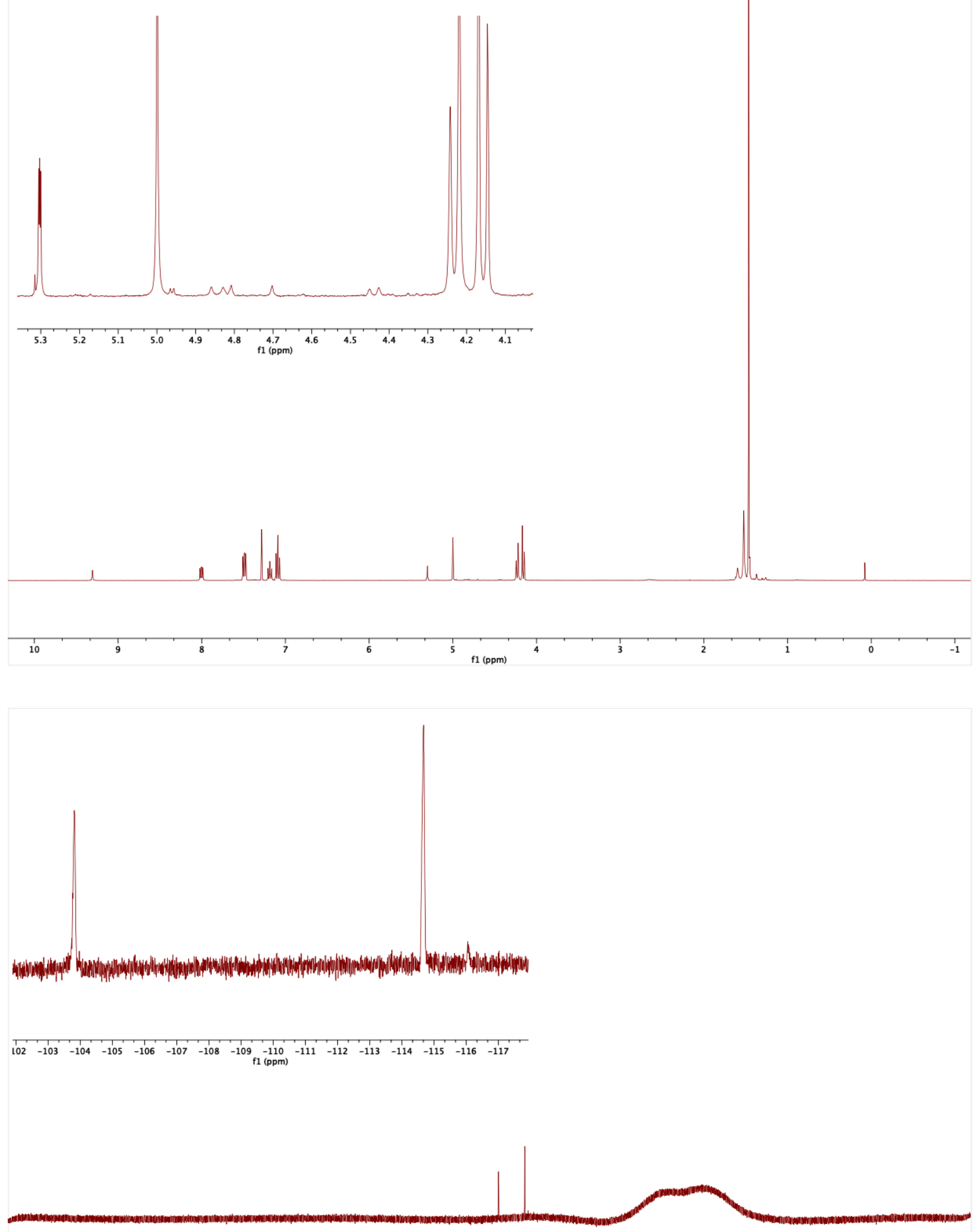